Copyright

by

Andrew Craig Tollison

2013 
The Dissertation Committee for Andrew Craig Tollison certifies that this is the approved version of the following dissertation

\section{Stereotype Threat in Male Nurse-Patient Interactions}

Committee:

Matthew McGlone, Supervisor

Erin Donovan-Kicken

Madeline Maxwell

Aaron Rochlen

Graham McDougall 


\title{
Stereotype Threat in Male Nurse-Patient Interactions
}

by

Andrew Craig Tollison, B.S.; M.S.

\author{
Dissertation \\ Presented to the Faculty of the Graduate School of \\ The University of Texas at Austin \\ in Partial Fulfillment \\ of the Requirements \\ for the Degree of \\ Doctor of Philosophy
}

The University of Texas at Austin

May 2013 


\section{Dedication}

This dissertation is dedicated to my parents. It is impossible to list everything you all have done for me throughout my life; however, there are two specific moments I will always cherish. Mom- I'll never forget the summer you coached my Little League team. We had fun! Talk about gender stereotypes, you may still be the only woman to ever coach Karns Little League baseball. Dad - I'll always remember how you convinced your golf buddies (most likely still drunk from the night before) to wake up extremely early on a golf trip at the beach so that you could make it back to my afternoon baseball game. When we pulled into the parking lot, there you were. You all have done so much for me, but these two moments really stand out to me. In both of these instances, you didn't have to say a word - your actions spoke much louder. Without your unconditional love and support, I would not be the person I am today. Every crazy idea I approached you all with, you were behind $100 \%$. Thank you for that. Since this is the only dissertation I plan to write and I dedicated it to you, you are now obligated to read the entire thing. Enjoy! 


\section{Acknowledgements}

I learned very early on in my graduate education that a Ph.D. is not an individual event nor is it a sprint. Instead, it is a marathon that requires the collaborative efforts of many people. I have had the opportunity to meet many wonderful people at Texas that helped contribute to my successes and assisted in me overcoming my failures. First and foremost I must acknowledge the contributions of my advisor, Matt McGlone. I cannot thank you enough for your support over my time at the University of Texas. Without your advising, encouragement, hours editing my work, and telling me to relax, I would not be the scholar I am today. I also owe a great deal of gratitude to Erin DonovanKicken for her guidance over the years. You provided me numerous invaluable opportunities to assist in research. I am very thankful I got that email right after you moved to Austin asking if I would be your Research Assistant. Also, to Madeline Maxwell, thank you for your thoughtful input and direction throughout my time at Texas. I also thank you for the opportunity to work the camp for two summers - what an exhausting and rewarding experience! To Aaron Rochlen and Graham McDougall, thank you both for your willingness to serve on my committee and your constructive feedback throughout the process. You both could have easily declined to serve on my dissertation committee since we are not in the same department, but you didn't. You both brought a depth of specialized knowledge that contributed greatly to this project. In addition to my dissertation committee, I have to acknowledge Susan, Jennifer, and Deanna. Without your assistance over the years on class-related issues to life advice, I'm not sure I would be where I am today. You all are genuinely wonderful people that I have enjoyed getting to know.

During my time in Austin, I have made a lot of wonderful friends and created many lasting memories. To my UA9 officemates, I'll never forget our "study breaks" playing bocce ball, Madden on Sega, or strategizing upcoming IM and kickball games. While I didn't necessarily get a lot of work done in the office, you all strongly influenced my research and teaching based on daily conversations that challenged me to think differently. To the Whales, it was an honor to be your coach for two years. I'll never forget Friday nights at Zilker and Gillis. Lastly, I will forever remember football Saturdays, Sunday Night BBQ, trips to Lake Travis, and the all left-handed golf outings.

To my family, you all have been there for me since Day 1. It is comforting knowing that I can call on you for anything. While unorthodox, I also want to thank my dog, Roxy, for her pseudo-support during this dissertation process. She literally sat right next to me as I wrote every word of this dissertation. Most importantly, she taught me a lot about stereotypes (Roxy is a well-behaved pitbull rescue) and never let me forget to get some fresh air. Last but not least, Shannon, thank you for experiencing Austin with me. I'm extremely proud of all your accomplishments and your service to this country. In closing, my degree is much more than classes and a dissertation. It is about the lasting friendships and experiences I had during this highly influential period in my life. That is why Austin will always hold a special place in my heart and why I'm proud to be a Longhorn. 


\title{
Stereotype Threat in Male Nurse-Patient Interactions
}

\author{
Andrew Craig Tollison, Ph.D. \\ The University of Texas at Austin, 2013
}

Supervisor: Matthew McGlone

Throughout history, men have played a significant role in the field of nursing. However, they currently represent only $12 \%$ of nursing students and $9.6 \%$ of Registered Nurses. As a minority in their occupation, male nurses experience gender-based barriers and negative stereotypes that female nurses do not. Research has addressed these barriers and stereotypes, but has lagged in identifying the consequences of exposure to them other than men's reluctance to enter or stay in the field. With the nurse-patient relationship at the core of quality healthcare, it is important to further clarify the consequences of stereotype exposure for male nurses in the clinical setting. The purpose of this dissertation was to investigate the communicative consequences of exposure to genderbased stereotypes among male nurses through the theoretical lens of stereotype threat (Steele \& Aronson, 1995). Stereotype threat describes the apprehension individuals experience from the prospect of confirming a negative self-relevant stereotype.

In the reported experiment, the salience of gender-based stereotypes (women are more empathic than men; men are better managers than women) was controlled for while 
male and female nursing students completed a simulated nurse-patient interaction task. Findings from this study highlight the influence of subtle stereotyping in simulated nursepatient interactions. Specifically, men were less grammatically accurate when participating under conditions of threat than when not under conditions of threat. This finding provides a need for caution as healthcare is experiencing a shift towards e-health, which will rely heavily on both the spoken and written word. Additionally, findings suggest that the disruptive potential of making salient stereotyped attributes (e.g., empathy) may be offset by patient gender. For example, male participants were less tentative under conditions of threat with a female patient than a male patient. The influence of patient gender provides optimism that a shift in gender-based stereotyping is occurring. Therefore, it is an optimal time to increase recruitment and retention efforts of men in the field of nursing. Recommendations for such efforts are offered as well as future directions for stereotype threat research in interpersonal communication and healthcare. 


\section{Table of Contents}

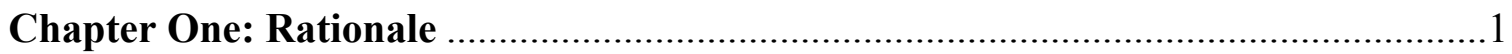

Introduction of Research Topic .......................................................................

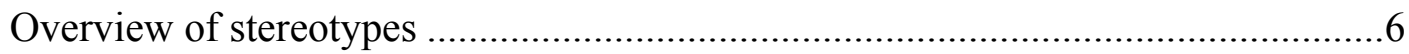

Research Goals.............................................................................................

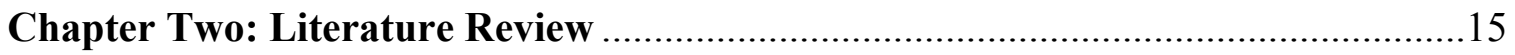

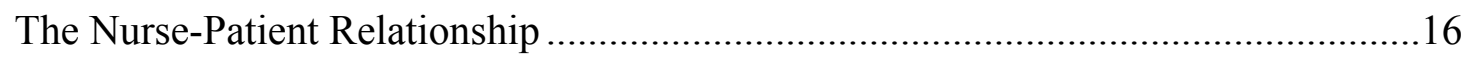

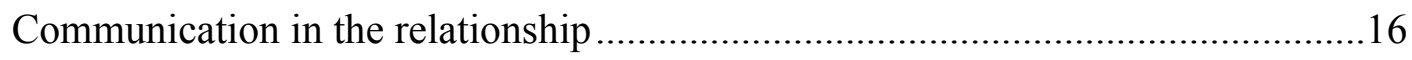

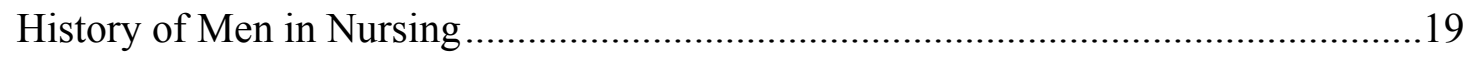

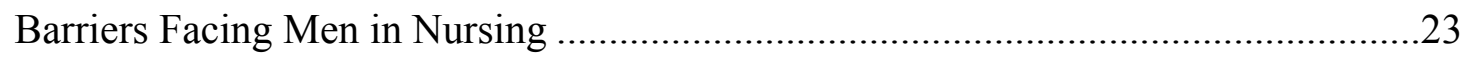

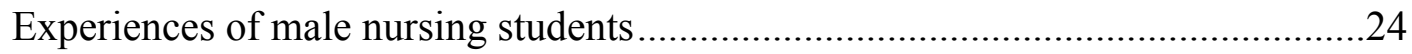

Gender-based barriers in nursing school...................................................27

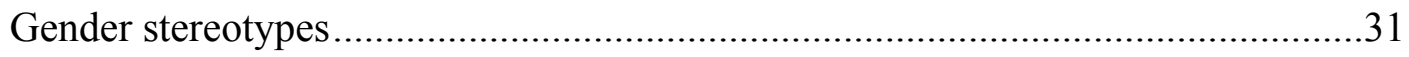

Gender issues associated with nursing .......................................................33

Gender issues associated with other female-dominated professions .................37

Research Influencing Stereotype Threat ..................................................... 40

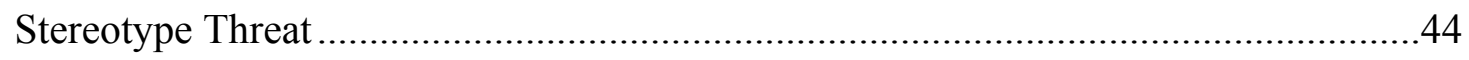

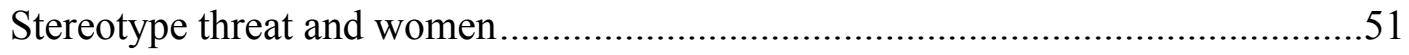

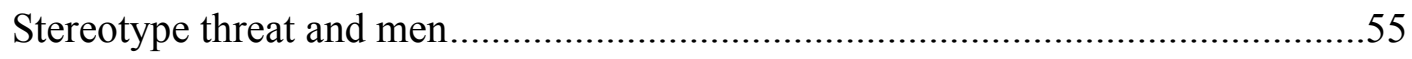

Stereotype Threat and Communication ........................................................60

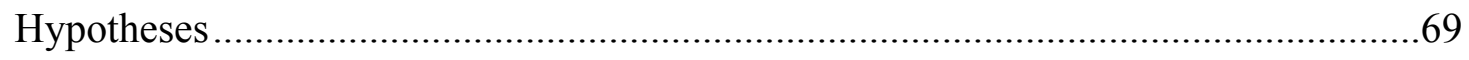

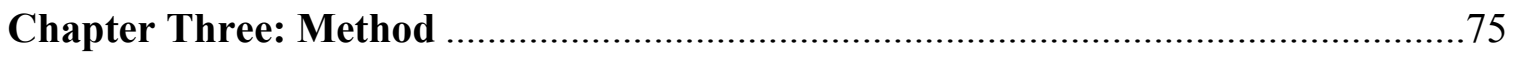

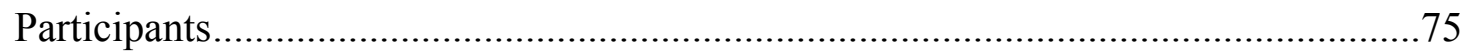

viii 


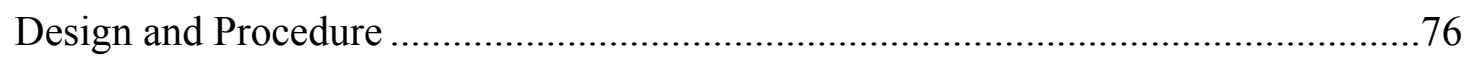

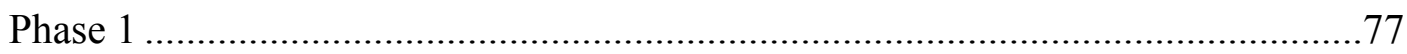

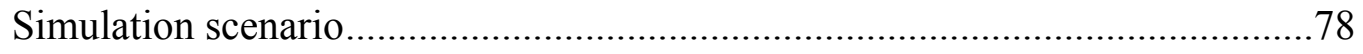

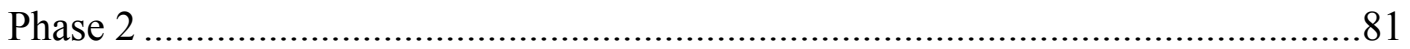

Personal report of communication apprehension...................................................82

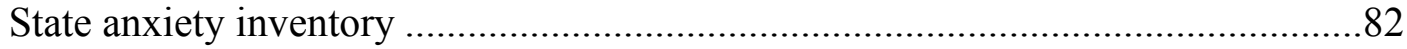

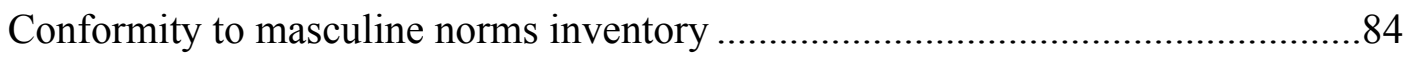

Conformity to feminine norms inventory ..............................................................85

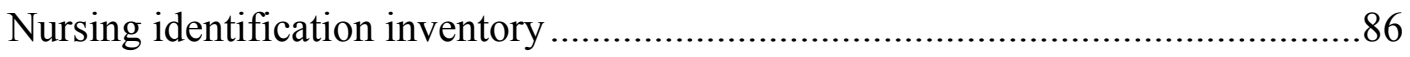

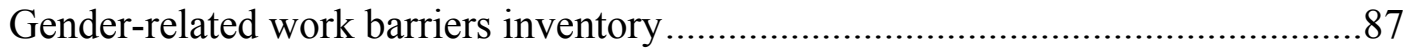

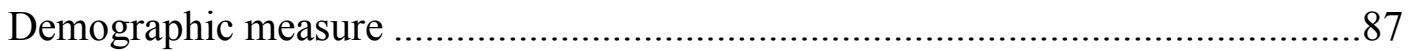

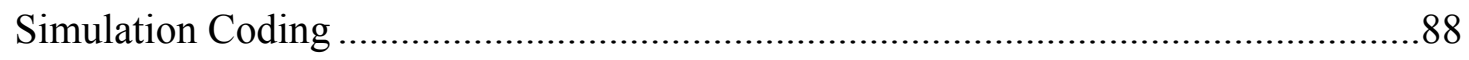

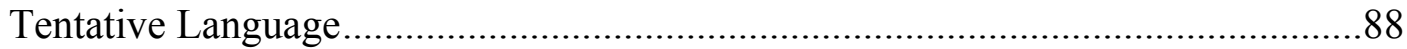

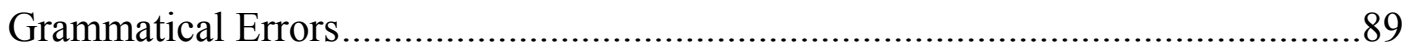

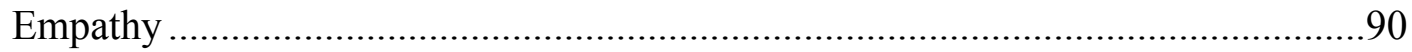

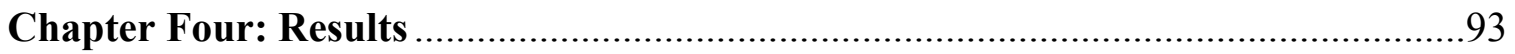

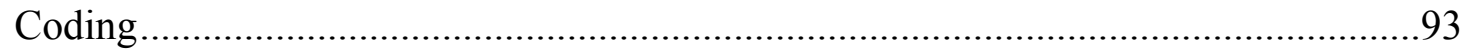

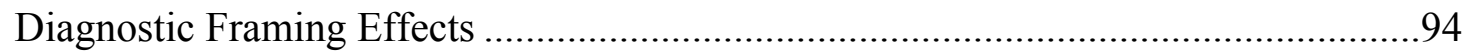

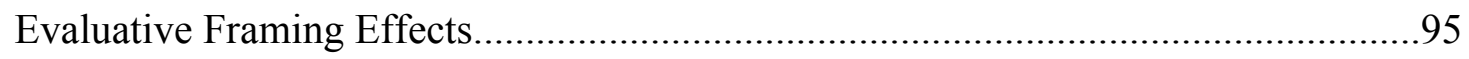

H1: Empathy Framing and Male Participants...........................................................95

H2: Patient Management and Female Participants .................................................96

Multiple Mediation Analysis ..................................................................................97 


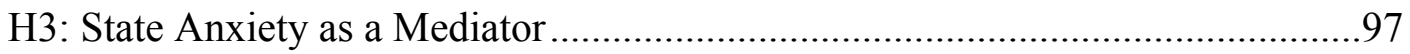

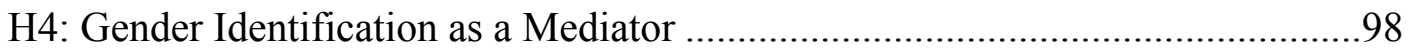

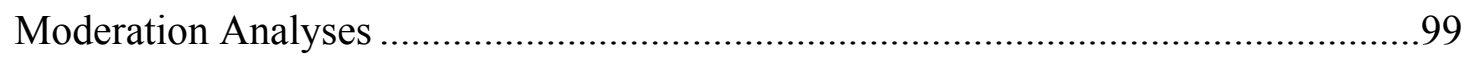

H5: Nursing Identification as a Moderator .........................................................99

H6: Perceived Gender-Related Work Barriers as a Moderator ........................... 100

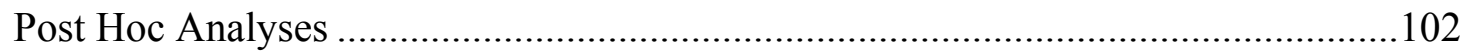

Post Hoc 1: Male Participants and Patient Gender ............................................. 102

Post Hoc 2: Female Participants and Patient Gender..........................................103

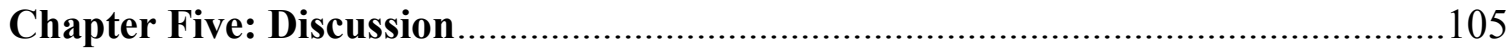

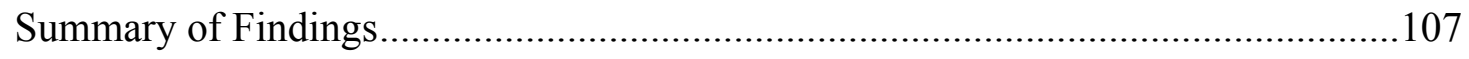

Limitations and Future Directions ................................................................. 120

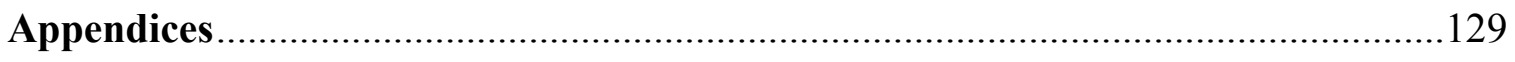

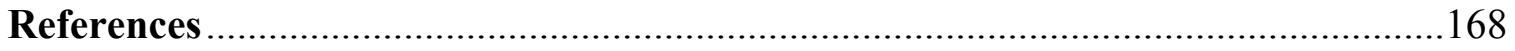

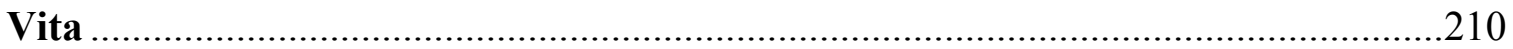




\section{List of Tables}

Table 1. Demographic profile of the study sample.

Table 2. Histogram of the Gender-Related Work Barriers Inventory.

Table 3. Histogram of the Gender-Related Work Barriers Inventory.

(Male Participants)

Table 4. Histogram of the Gender-Related Work Barriers Inventory. (Female Participants)

Table 5. Participant Cell Distribution Across Participant Gender,

Patient Gender, and Diagnostic Frame

Table 6. Mean (SD) Rates of Tentative Language and Grammatical

Errors by Participant Gender, Patient Gender, and Diagnostic Frame

Table 7. Mean (SD) Rates of Tentative Language and Grammatical

Errors by Patient Gender for Male Participants (Post Hoc Analysis)

Table 8. Mean (SD) Rates of Tentative Language and Grammatical 136

Errors by Patient Gender for Female Participants (Post Hoc Analysis)

Table 9. Correlation Matrix of GRWB, CMNI, \& CFNI 


\section{List of Figures}

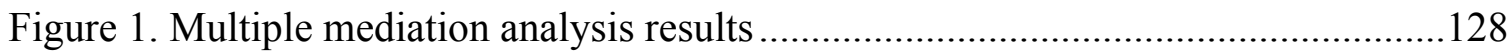




\section{Chapter One:}

\section{Rationale}

Jack Byrnes: Greg's in medicine too.

Bob Banks: What field?

Greg Focker: Nursing.

Bob Banks: Ha ha ha ha. No, really, what field are you in?

Greg Focker: Nursing.

(Meet the Parents, 2000)

\section{Introduction of Research Topic}

Not all professions are equally welcoming to men and women. In outlining a rationale for these gender-based occupational inequalities, a considerable body of literature suggests that individuals make stereotypical judgments regarding gender and professions, a phenomenon known as occupational stereotyping (Deaux, 1995; White \& White, 2006). This stereotyping is predicated on the assumption that particular occupations are best suited for specific attributes attached to gender (Holland, 1973), and these stereotypes make it difficult for individuals to enter into non-traditional occupations. As reported by Lupton (2006), men who enter a female-dominated profession face threats to their masculine identity, an identity most men work hard to maintain. Of specific interest to this project are men entering the female-dominated profession of nursing. Issues addressed in this project include the gender-based barriers and stereotypes faced by male nurses and the impact these barriers and stereotypes have on the communicative performance of male nurses in the nurse-patient relationship.

There is consensus among healthcare practitioners that the nurse-patient relationship is at the core of efficient and quality patient care (Peplau, 1952, 1987, 1997). Unfortunately, the amount of time nurses spend with patients is trending towards that of 
physicians - limited. Although nurses comprise the largest population of healthcare providers in the United States (Bureau of Labor Statistics, 2012), that population is aging. While there has been a noted increase over the past decade in the number of new nurses entering the workforce under the age of 26 , it is still projected that the average age of the registered nursing workforce in 2015 will be 44.1 years old, which is right under the all time high of 44.2 years of age in 2012 (Auerbach, Buerhaus, \& Staiger, 2011). As the nursing population ages and retires, there are simply not enough new nurses to meet staffing needs. The United States Department of Health and Human Services Health Resources and Services Administration (2006) published a projection of a 20\% nursing shortage by 2020 , which equates to a conservative estimate of 400,000 vacant nursing positions in the United States.

Focusing on nurse retirement rates, there are not enough students graduating from nursing programs to fill these vacant positions. With the demands of healthcare consumers on the rise, the looming nursing shortage will have a negative impact on healthcare by decreasing patient access to medical practitioners while also reducing the overall quality of care. For those hospitals that currently report low nurse-to-patient ratios, there have been noted decreases in patient care, with nursing burnout and an overall lack of job satisfaction being commonly cited by the nurses themselves (Aiken, Clarke, Sloane, Sochalski, \& Silber, 2002). A report by the Joint Commission on Accreditation of Healthcare Organizations (2007) noted that decreased nursing staff levels were a significant contributor to approximately $24 \%$ of unanticipated patient deaths, injury, and permanent loss of function. With problems of this magnitude, more steps need to be taken to reduce nursing burnout and increase job satisfaction. Increasing 
nursing staff levels could provide patients with more nurse face-time, which in turn has the potential to produce more positive patient outcomes. Although recent recruitment efforts have been aimed at men and ethnic minorities (Davis \& Bartfay, 2001), the overall nursing workforce is still extremely homogenous with Caucasian females making up a significant proportion (70.3\%) of the nursing workforce (D'Antonio \& Whelan, 2009; O’Lynn, 2004). Yet this homogenous group of healthcare workers is providing care for a more gender-balanced and increasingly ethnically diverse population.

Despite nursing being listed as a top occupation for job growth potential, men only account for $12.1 \%$ of the nursing student population (American Association of Colleges of Nursing, 2006; National League for Nursing, 2007). This low percentage is attributable in equal parts to the low numbers of men who enroll in nursing curricula as well as the high attrition rates among men who do enroll. For men that do enroll in collegiate nursing courses, $40-50 \%$ are expected to drop out, fail, or transfer to a nonnursing course (Mulholland, Anionwu, Atkins, Tappern, \& Franks, 2008; Poliafici, 1998; Pryhmachuk, Easton, \& Littlewood, 2008; Villeneuve, 1994; Wilson, 2005). This tendency contrasts with the attrition rate of women, which is $17-25 \%$ (Finlayson, Dixon, Meadows, \& Blair, 2000; Godfrey, 2000; Lipley, 2000). Commonly cited explanations for the higher male attrition rates are the lack of male faculty role models, a decreased male student-body presence, and gender-biased language in the classroom as well as course reading materials (Bell-Scriber, 2008; Gardner, 2005; O'Lynn, 2004; O’Lynn \& Tranbarger, 2007; Smith, 2006). These obstacles pose distinct challenges to male students that their female counterparts do not face. Moreover, men who do graduate from nursing programs and enter the workforce are significantly more likely than women to 
quit nursing within four years of graduation. Yurkovich (2006) found that 7.5\% of men quit nursing within the four-year post-graduation span, compared to only $4.1 \%$ of women quitting in the same time frame. As it currently stands, approximately $9.6 \%$ of professional nurses are men (U.S. Census Bureau, 2013). At previous points in history (e.g., Hippocratic period in ancient Greece, The Crusades), men made up a majority of the nursing workforce and were looked to as the nursing standard (Christman, 1988; O'Lynn, 2004). In the last several decades, however, their status in the field is as a minority not preferred by most patients (Chur-Hansen, 2002; Kerssens, Bensign, \& Andela, 1997). Despite this minority status, men are slowly gaining some traction in the nursing profession. For example, the current male nursing population has already doubled the previous low of 4\% in 1992 (Burt, 1998). Research by Yang et al. (2004) attributes this growth to the potential for men to specialize in specific areas of nursing such as psychiatry, mental health, surgery, and emergency/intensive care units.

Despite gaining more social acceptance for specialized areas of nursing, men still face significant social barriers that female nurses do not. Although there is consensus among researchers that nursing quality does not hinge on gender (e.g., Lo \& Brown, 1999), the general public's perception of nurses is less enlightened. In society's view, men are perceived to be aggressive, unaffectionate, and control-oriented - all attributes not commonly associated with nursing (Aries, 1996; Flannery, 2000). Nurses, on the other hand, are expected to possess empathic, nurturing, and helping qualities - attributes strongly associated with women (Deaux, 1995). In fact, White and White (2006) classify female and nurse as a congruent pairing under the social microscope, but male and nurse as incongruent in society. By no means does this imply that men are incapable of being 
nurturing and caring, but rather supports the notion that men who demonstrate such characteristics are in the minority (McDougall, 1997). Thus, the overall lack of men in the field only further supports the salience of society's gender-related stereotype impugning men's capacity to be nurses.

In addition to the stereotype that successful nurses are women with superior empathy and nurturing capacities, the stereotype that male nurses tend to be gay is problematic to the recruitment and retention of heterosexual men. Regardless of sexual orientation, men who choose nursing as a profession are at an increased risk of being perceived as gay by society (Harding, 2007; Loughrey, 2008). Such social perceptions have the potential to create a psychosexual conflict for heterosexual male nurses, prompting them to either not pursue nursing or to overtly "act out" their heterosexuality if they do choose to enter nursing. Male nurses who attempt to act out their masculinity are at risk of providing poor quality of care (Lupton, 2006; O'Neil, 1981a, 1981b). In addition to being stereotyped as homosexual, male nurses also face the stereotype of having failed out of medical school and not being trustworthy to provide quality care (Hart, 2005; Loughrey, 2006). Given that men are faced with a significant amount of stereotypes associated with their choice of profession on a regular basis, their desire to enter, stay, and provide quality care is compromised.

The history of male nurses as an occupational minority and the prejudices they face professionally provide the backdrop for the investigation of nurse communication skills for this dissertation. Specifically, I will investigate the influence of "stereotype threat" (Steele \& Aronson, 1995) on male nurses' performance of these skills. 
Overview of stereotypes. In the decades since communication theorist Walter Lippmann (1922) first described people's generalizations about groups as “stereotypes," scholars in a variety of disciplines have documented their pervasive influence in social thought and interaction. Traditionally this research has investigated how social stereotypes - that is, knowledge structures containing beliefs and expectations about the typical members of social groups (Stangor, 2009) - distort one group's perceptions of another. For example, communication scholars have studied the impact of stereotypes on Whites' evaluations of Black crime suspects in television news (Dixon, 2006; Domke, McCoy, \& Torres, 1999; Entman \& Rojecki, 2000; Gilliam \& Iyengar, 2000; Oliver, 1999; Oliver \& Fonash, 2002; Peffley, Shields, \& Williams, 1996), the effects of opposite-sex speech style stereotypes on interactions between men and women (Berryman \& Wilcox, 1980; Giles, Scholes, \& Young, 1983; Kramer, 1977), and how age stereotypes lead younger people to overaccomodate in communication with older people (Hummert, Shaner, Garstka, \& Henry, 1998; Ryan, Giles, Bartolucci, \& Henwood, 1986).

In recent years, the focus of stereotyping research has shifted from the behavioral correlates of endorsing stereotypes to the cognitive consequences of being targeted by them (Aronson \& McGlone, 2009). This research focus was anticipated by social psychologist Gordon Allport, who noted that a possible side effect of belonging to a group targeted by negative stereotypes is an inadvertent inclination to confirm these stereotypes. Katz and colleagues (Katz \& Benjamin, 1960; Katz \& Cohen, 1962; Katz \& Greenbaum, 1963; Katz, Roberts, \& Robinson, 1965) initially explored Allport's claim in the classroom context. Katz, Goldstone, \& Benjamin (1958) found in mixed ethnic 
groups of middle school students performing problem-solving tasks that White students initiated more interactions than Black students, and both White and Black students talked more to White students than Black students, even though participants were matched for intelligence based on IQ test performance a priori. Later, Katz and Benjamin (1960) found that the relationship between intelligence-matched Black and White students, when engaged cooperatively in problem-solving, exhibited the disparity predicted by prevailing social stereotypes, in that White students were more active, influential, and accurate in problem-solving than the Black students. Katz and Benjamin coined the term "interracial interaction disability" to represent this state of affairs. However, contemporary research on the "stereotype threat" (Steele \& Aronson, 1995) phenomenon indicates that this phenomenon generalizes to stereotypes about ascribed characteristics other than race.

Steele and Aronson (1995) coined the term "stereotype threat" to describe the apprehension people experience from the prospect of confirming a negative self-relevant stereotype. A variety of cultural stereotypes attribute dispositional deficits to certain groups ("women can't do math," "Black people aren't intelligent," "elderly people have poor memories," etc.), and group members are as aware of the stereotypes as anyone in the culture, whether or not they personally agree with them. In situations where a devaluing stereotype is relevant (e.g., a mathematics classroom), people targeted by the stereotype (e.g., women) experience an extra mental burden stemming from concern that certain behavioral outcomes (e.g., poor test performance) will reinforce the stereotype in the eyes of others. Once initiated, stereotype threat produces a number of disruptive effects, including decreases in intellectual performance (Steele \& Aronson, 1995; Spencer, Steele, \& Quinn, 1999) and increases in physiological arousal (Ben-Zeev, Fein, 
\& Inzlicht, 2005; O'Brien \& Crandall, 2003). Over time it may prompt defensive adaptations such as disengagement from domains of intellectual activity (e.g., mathematics education) where the stereotype is relevant, and consequently retard development in these domains. Thus the phenomenon can result in a self-fulfilling prophecy whereby people come to resemble the very stereotype they feared confirming in the first place.

More than 300 published studies in the years since Steele and Aronson's (1995) initial investigation have documented the pernicious effects of stereotype threat in a variety of populations and testing domains (for a comprehensive review, see Aronson \& McGlone, 2009). These effects have been demonstrated in the performance of women on mathematical reasoning (Inzlicht \& Ben-Zeev, 2000; McGlone \& Aronson, 2006; Spencer, Steele, \& Quinn, 1999) and political knowledge tests (McGlone, Aronson, \& Kobrynowicz, 2006), Latinos (Gonzalez, Blanton, \& Williams, 2002) and AfricanAmericans and Native Americans (Osborne, 2001) on college preparedness tests, low SES students on intelligence tests (Croizet \& Claire, 1998), and the elderly on memory tests (Levy, 1996; Hess \& Hinson, 2006). Although stereotype threat is most keenly experienced among groups historically targeted by negative stereotypes, it is a predicament that can beset anyone, because any group can be compared to another reputed to be at an advantage on some dimension (Aronson, 2002). Thus it can impair the performance of even those groups who are neither minorities nor commonly stereotyped as having an ability deficit. White male university students, for example, bear no historical stigma associated with intelligence. However, in some circumstances comparisons with allegedly superior groups arise, thereby creating a situational threat. 
Aronson et al. (1999, Experiment 1) created such a threat when they gave White male undergraduates at Stanford University a challenging math test and told half of them their scores would be compared to those of Asian students. Mention of the intended comparison made students mindful of the stereotype of Asian mathematical superiority and consequently impaired their performance relative to others who were not told that this comparison would be made. The impairment occurred despite the fact that the White male students in the study had good reason to be confident in their math skills - most were math or engineering majors with SAT math scores over 700. Similarly, students enrolled in the honors program of a public university were observed to perform at a decreased level on a test of spatial reasoning when led to believe they would be compared to students at a prestigious private university nearby (McGlone, Kobrynowicz, \& Aronson, 1999). Such studies refute any claim that stereotypes have impact only on those who have faced broad discrimination or prejudice, or those who harbor persistent self-doubts about their group's abilities. Under certain circumstances, anyone is prone to performing poorly when confronted by a stereotype that casts their group as being at a disadvantage.

\section{Research Goals}

In this dissertation, I investigate the impact of gender-based stereotype threat on male nurse's performance in a simulation of nurse-patient communication skills. Prevailing sociocultural stereotypes portray men as less emotionally expressive, sensitive, and empathic than women (Aries, 1996; Broverman, Vogel, Broverman, Clarkson, \& Rosenkrantz, 1972; Eagly \& Steffen, 1984; Flannery, 2000; Spence, Helmreich, \& Stapp, 
1974). Stereotype threat researchers have demonstrated that reminding men of these stereotypes can impair their performance in interpersonal perception tasks. For example, Koenig and Eagly (2005) investigated men's abilities to correctly interpret expressive behaviors of others. Participants were placed in either a threatening or non-threatening condition, with participants in the threat condition being explicitly told that the test was a measure of social sensitivity where "men do worse than women." In contrast, those in the non-threat condition were told the test was a measure of complex information processing ability with the statement "men do worse than women" being completely omitted.

Results from their study indicated that men in the threat condition were significantly less accurate in their interpretations than those in the non-threat condition. On the other hand, women experienced a slight lift in the threat condition. In a separate study focused on interpersonal sensitivity and emotional expressiveness, Leyens, Desert, Croizet, and Darcis (2000) had men and women complete a lexical decision task in which participants were told they were taking part in an academic exercise (non-threatening condition) or in a task aimed at understanding gender differences in how affective information is processed (threatening condition). Participants in the threat condition were told "it is a well-known fact that men are not as apt as women to deal with affect in general and specifically to process affective information as effectively." During the experiment, participants were shown a target word and had to indicate if that word referred to an emotion or not. Results indicated that under threat, men made significantly more errors in classifying affective versus non-affective words. Specifically, men in the threat condition appeared to overinclude words in the affective category to disconfirm the 
stereotypical deficiency attached to men and emotional sensitivity. In both of these studies, no significant differences were reported for female participants across conditions.

The two aforementioned investigations are critical to the current body of stereotype threat literature because they sought to demonstrate the influence of stereotype threat on a social group - men - traditionally considered to be non-stigmatized. In these studies, men were aware of the social stereotype that they are not as emotionally tuned as women, and making this stereotype salient had a detrimental influence on their task performance. If men are aware of their socially defined limitations, under what additional conditions are they vulnerable to the pernicious effects of stereotype threat? One goal of this dissertation was to investigate the influence of society-based gendered stereotypes associated with nursing. Given men's minority status in the nursing profession, male nursing students would be expected to exhibit performance deficits related to stereotype threat when nursing is framed as an activity requiring high empathy ability. Stereotype threat is elicited when participants are placed in a situation they believe can diagnose their amount of some impugned ability - in this case, empathy. Therefore, it is hypothesized that male participants in the threat condition (equating patient interaction with empathic ability) will exhibit a decrease in performance when compared to others for whom the activity is framed as diagnosing a non-impugned ability (equating patient interaction with management ability) or a control condition with no ability framing. The current study diverges significantly from extant stereotype threat research in that it seeks to measure the threat's impact on communicative behavior, not a test score per se - the second goal of this dissertation. 
Although the vast majority of stereotype threat studies have focused on academic and intellectual performance deficits, recent research has explored threat effects on communication performance in evaluative contexts (Breckinridge, Tollison, \& McGlone, 2011; McGlone \& Pfiester, in press). McGlone and Pfiester (in press) sought to examine the effects of gender-based stereotype threat on the communication performance of college undergraduates. They presented participants with an interpersonal conflict simulation in which participants were asked to help the involved parties resolve their dispute by offering advice and suggestions. Prior to participating in the simulation, participants were told the simulation was diagnostic of differences in leadership ability (a stereotypically male-typed ability) or of one's ability to build and maintain personal relationships (a stereotypically female-typed ability). For a third control group, the simulation was not framed as diagnostic of any ability. Across all three conditions, participants were told that their results would be used to investigate gender differences in performance. Participant simulations were recorded and analyzed for speech disfluency (e.g., vocalized pauses) and tentative language (e.g., hedges) use. Findings indicated that both women and men were less fluent and more tentative in their language when placed in a threat condition as compared to those not under conditions of threat. For women, this was being told the experiment was a measure of leadership ability and for men, being given the instructions that the experiment was used to measure relational maintenance served as the threat prime. In explaining their findings, McGlone and Pfiester (in press) noted that the decrease in communication performance was due to the increase in state anxiety under conditions of stereotype threat. McGlone and Pfiester's findings 
demonstrate the impact stereotype threat can have on the communicative behaviors of individuals in evaluative contexts.

This dissertation employed a variant of McGlone and Pfiester's (in press) experimental paradigm to investigate the influence of stereotype threat on male nursing students' communication performance in an evaluative context that places an emphasis on the nurse-patient relationship. Based on the stereotype threat framework, when a member of a stigmatized group is faced with a negative self-relevant stereotype and is fearful of confirming that stereotype, they are presumed to experience psychological discomfort (Steele \& Aronson, 1995). As will be further explicated in the subsequent literature review, this discomfort can impair an individual's communication performance and in so doing render the performance as appearing to "confirm" the negative stereotype. When thinking about stereotyped and stigmatized populations in the healthcare profession, the male nurse is almost always one of the first to come to mind (Evans, 2002). Whether commonly present in a healthcare setting or not, most people are aware of this stereotype as it is commonly on display in popular movies and television. One need look no further than the outlined scene from Meet The Parents at the beginning of this chapter. To the characters in the movie, it is highly comical that a man would actively choose to be a nurse. Building on this social stereotype that men are not nurses, I measured the influence of stereotype threat on the communicative performance of male and female nursing students during a brief simulated patient interaction. This simulation was conducted online, which leads to the third and final goal of this dissertation assessing the use of online data collection in stereotype threat research, a data collection method that has yet to be utilized. 
In the chapter that follows, I provide a review of relevant literature motivating the hypotheses to be tested and the method used in this study. I first offer a brief review of research about the nurse-patient relationship before transitioning to the history of male nurses. In reviewing this history, specific barriers and stereotypes faced by male nurses are addressed. I then review the history of stereotype threat research since Steele and Aronson's (1995) seminal work, with an added emphasis on significant findings that extend beyond the commonly cited effects in the domain of academic standardized testing. Finally, I review the limited literature base that has focused on men under stereotype threat, as well as the evaluative context of communication behavior. 


\section{Chapter Two:}

\section{Literature Review}

For over four decades, healthcare researchers have investigated patient health outcomes that derive from their relationships with providers, such as patient satisfaction (Di Palo, 1997; Fox \& Storms, 1981; Korsch, Gozzi, \& Francis, 1968; Ware, DaviesAvery, \& Stewart, 1978), physiological outcomes (Greenfield, Kaplan, \& Ware, 1985; Kaplan, Greenfield, \& Ware, 1989; Kinnersley, Stott, Peters, \& Harvey, 1999; Selfe, Matthews, \& Stone, 1998), adherence to prescribed medications and routines (Blackwell, 1996; Garrity, 1981; Golin, DiMatteo, \& Gelberg, 1996; Ley, 1982; Roter, 1989), reduction of malpractice claims (Hickson, Clayton, Githens, \& Sloan, 1992; Lester \& Smith, 1993; Penchansky \& Macnee, 1994; Vincent, Young, \& Phillips, 1994), and office visit frequency and duration (Arborelius \& Bremberg, 1992; Greenfield, Kaplan, Ware, Yano, \& Frank, 1988; Henbest \& Fehrsen, 1992; Marvel, Doherty, \& Weiner, 1998). One significant commonality across these various literatures is the importance of effective patient-provider communication as a means of bolstering quality health care by addressing system and patient-level disparities. Effective healthcare providers elicit patient opinions and narratives about their current health conditions (Cortes et al., 2009). Given the amount of time nurses interact with patients, relative to other healthcare providers (i.e., physicians), they are in the best position to establish rapport with patients and maintain the two-way communication channel researchers deem essential to quality healthcare (Campbell, 1984; Fleischer et al., 2009; Hagerty \& Patusky, 2003; Hartrick, 1997; Paterson \& Zderad, 1976; Peplau, 1987; 1997; Ramos, 1992). 


\section{The Nurse-Patient Relationship}

Registered nurses are the largest healthcare provider population in the United States, with 2.6 million registered nurses (Bureau of Labor Statistics, 2012). However, the overall number of registered nurses is steadily declining from an all-time high of 2.9 million registered nurses in 2004 (United States Department of Health and Human Services, Health Resources and Services Administration, 2006). There is a projected nursing shortage of $20 \%$ by 2020 in the U.S., which will yield a need for over 400,000 registered nurses (United States Department of Health and Human Services, Health Resources and Services Administration, 2006). A major factor contributing to this shortage is the age of the current registered nursing population. In 2004, approximately $8 \%$ of the registered nursing workforce was under the age of 30 (Norman et al., 2005), in contrast to $25.1 \%$ of registered nurses under 30 in 1980 (Spratley, Johnson, Sochalski, \& Spencer, 2002). This trend steadily increased until the mean age of registered nurses reached 44.2 years of age in 2012 (Auerbach, Buerhaus, \& Staiger, 2011). As nurses continue to age and retire, greater efforts need to be made to recruit younger applicants into the registered nursing pool. With the demands of healthcare consumers increasing, the overall lack of registered nurse staffing will decrease access to quality health care. Aiken, Clarke, Sloane, Sochalski, and Silber (2002) observed that registered nurses employed at hospitals with low nurse-to-patient ratios experienced increased rates of burnout and overall job dissatisfaction, which in turn were associated with lower quality healthcare

Communication in the relationship. The nurse-patient relationship is at the core of nursing (Peplau, 1952, 1987, 1997), as nurses play a fundamental role in the delivery 
of quality patient care (Barrere, 2007; Paterson \& Zderad, 1976; Sheldon, Barrett, \& Ellington, 2006; Wilkinson, Roberts, \& Aldridge, 1998). One reason this relationship is of such importance is the amount of time nurses spend interacting with patients relative to physicians (Sheldon et al., 2006). Physician-patient interactions are commonly rushed, technically-oriented, and unidirectional (Ford, Fallowfield, \& Lewis, 1996). Nurses, on the other hand, have the benefit of extended patient interactions and thus often become the "point people" for patients. These interactions, for both nurses and physicians, extend beyond the confines of a physician's office, as there has been a recent increase in the amount of patient communication occurring electronically (Moyer et al., 2002; Sittig, King, \& Hazlehurst, 2001). With patients gaining access to their healthcare providers asynchronously, it is necessary for providers to focus on the delivery of the message so that they may meet the needs of their patients. Focusing on nurse and patient roles in the relationship, Madden (1990) defined the nurse-patient relationship as:

a process in which both the patient and the provider are actively working toward the goal of developing patient health behaviours chosen for consistency with the patient's current health status and lifestyle; focusing on mutual negotiation to determine activities to be carried out toward that goal; and using a supportive and equitable therapeutic relationship to facilitate that goal (p. 85)

Based on this definition, the nurse and patient are equals within this relationship. This perception of equality has come under some academic and practitioner-based scrutiny since the nurse is the professional in the relationship (e.g., Fealy, 1995). Whether seen as an equal or a superior in the nurse-patient relationship, scholars are in agreement that it is important for patients to actively receive necessary information while also being given time to voice their own opinions and concerns (Campbell, Auerbach, \& Kiesler, 2007), 
actions that occur when patients are comfortable with their environment and more frequently occur in the presence of nurses than physicians.

Within the nurse-patient relationship, there is a sense of mutuality that influences the communication and interactions between nurses and patients. As noted by Henson (1997), nurses and patients develop "a feeling of intimacy, connection and understanding of another... a dynamic process characterized by an exchange between people to a common goal or shared purpose" (p. 79). Other scholars have followed this lead by including communication as an indispensable interpersonal component of nursing practice (Barrere, 2007; Finch, 2005; Rogan \& Timmins, 2004; Shattell, 2004). Fleischer et al. (2009) further support these claims by stating that superior interpersonal communication skills are a crucial component of nursing. In fact, it is the communication and interaction between nurses and patients that helps to facilitate these relationships, and without communication and interaction; these relationships would not be able to develop and thrive. In fact, a recent Gallup poll indicated that nurses are the most trusted occupation in society today - scoring an 84\% (Jones, 2011).

Although this previously cited research supports the importance and necessity of communication in the nurse-patient relationship, one cannot overlook the importance of accuracy in the delivery of necessary information provided to patients. There is no question that it is necessary for all healthcare providers - nurses included - to provide patients with accurate information related to their diagnosis, prognosis, and treatment in an easy to understand manner (Azoulay et al., 2000; Lister, 1971; Peeke, Monroe, \& Hulit, 1968). Despite having a foundational understanding of what components are necessary for effective and functional communication in the healthcare setting, limited 
attention has been paid to factors influencing the ways providers deliver information to their patients. Specifically, this project examines the population of male nurses to investigate how being in a strongly gender-typed profession that is at odds with one's own gender influences one's ability to communicate with patients. In the following section, a review of men's presence in the field of nursing is provided, with a specific focus on gender-based barriers and stereotypes.

\section{History of Men in Nursing}

When my 10-year old daughter was younger, she was embarrassed to tell her friends what her father did for a living. She knew she would be teased and of course she was right (Anonymous Male Nurse, Hart, 2005, p. 48).

Nursing is currently a female-dominated profession centered on the stereotypically feminine attributes of caring, empathy, and compassion (Deaux, 1995). These characteristics strongly align with the etymology of the word nursing, from the Latin word nutrio meaning "to nourish" (Geissler, 1986; Stewart \& Austin, 1962). Although men are not stereotypically perceived as nourishers, they have nonetheless played an integral role in the development of the nursing profession, as we know it today. The first nursing school was established in 250 B.C.E. in India and only accepted male students (Nelson \& Belcher, 2006; Wilson, 1997). In fact, it was common practice for early nursing programs to only accept male applicants. This academic dominance translated to the clinical setting. In the beginning, nursing duties were carried out predominantly by male clergy (Christman, 1998; Goodnow, 1940; O’Lynn, 2004). The first hospitals, which were established by the Roman Catholic Church, recruited monks and clergy to serve as the main caregivers. In addition to hospitalized nursing, medical 
care has always been a necessity in times of war. Women initially were not allowed on the battlefields, so despite being a lack of professional training, male soldiers served as field nurses for one another. This history precedes the first recognized nurse in the United States, Friar Juan de Mena $-\mathrm{a} 16^{\text {th }}$ century male monk (Nelson \& Belcher, 2006). Although men played an integral role in the development of nursing as a profession, they are infrequently mentioned in contemporary accounts of nursing's history. This omission in historical texts further perpetuates the notion that male nurses are a deviation from the norm (Okrainec, 1990).

From its infancy until the mid-1800s, nursing was a male-dominated profession as women were relegated to taking care of the home. However, during the $1800 \mathrm{~s}$, it became more acceptable for women to seek occupational opportunities outside of the home nursing being one of those occupational opportunities. As women transitioned into nursing care, the role of male nurses began a gradual shift. Instead of performing traditional nursing care, men became responsible for the intimate care of other men and pacifying the mentally ill (O’Lynn \& Tranbarger, 2007). This focus on mental health nursing being an appropriate occupation for men is still present today as men commonly gravitate towards this specialty. The final shift that fast-tracked nursing into being a female-dominated profession came in the form of the "mother of modern-day-nursing," Florence Nightingale. Nightingale introduced the feminine image into nursing, became an activist for professional change, and has been commonly linked to the demise of male nurses (Connolly \& Rogers, 2005). As a field nurse during the Crimean War, Nightingale earned the necessary credentials and respect to advocate for higher medical standards in hospitals (Donahue, 1996). She attributed subpar medical standards to the 
alleged inability of men to be organized and perform the necessary tasks that go along with being a nurse (Bullough, 1994). In a letter complaining about the routines of the hospital to Major Charles Sillery, Commandant and Chief Purveyor at the Scutari Barrack Hospital where Nightingale was working in 1855, Nightingale stated, "One woman does the work of more than a man in a hospital...for men are not accustomed to these duties..." (McDonald, 2010). In Nightingale's eyes, "every woman was a nurse" and women were more competent than men as nurses (Connolly \& Rogers, 2005, p. 46). Based on this ideology and her advocacy efforts, Nightingale established her own nursing school in England in 1860 and began to change the pattern of nursing by only admitting women. A strong shift occurred at this point as women were obtaining training from a credible institution, which made them more employable when compared to their male counterparts. To Nightingale, nursing was a completely natural profession for women but completely unnatural for men (Anthony, 2006), and she used this line of reasoning to break the stronghold men previously held in the nursing profession.

It was not until 1888, at Bellevue Hospital in New York City, that men were able to get a formal nursing education in the United States (Anthony, 2004). Although this was a step towards integrating men back into the nursing curriculum, men and women were completely segregated when it came to coursework. Women received training on bedside manners and "traditional" nursing, while the male curriculum emphasized specialties such as mental health and urology. In fact, men were prohibited from studying subjects such as maternal nursing and obstetrics. Not only did male students receive completely different training from women, they were also continually subject to psychological tests and had to maintain well-above average grades to remain in the 
program (Pittman, 2005). Perhaps the most absurd requirement for male nursing students was participation in sports, ostensibly to determine their sexual orientation. Administrators reasoned that if a man was unable to "hold his own" during sporting events, he might be a homosexual, which constituted grounds for immediate expulsion from a nursing school.

In addition to the hardships at the academic level for men pursuing nursing degrees, they were not always accepted into the profession itself. Founded in 1897, the American Nurses Association established bylaws that did not allow men to practice nursing until 1930 (Kalisch \& Kalisch, 1978). It was not until the 1950s that men were able to serve as nurses in the United States Army and Navy Nurse Corps, established in 1901 and 1908 respectively. The female nurses that served in these branches actively lobbied to keep men from being able to serve in this capacity. However, due to nursing shortages during wartime, the United States Army Nurse Corps bylaws were edited to allow men to serve as nurses, which began during the Korean War. In total, approximately $30 \%$ of military nursing corps are currently staffed by men (Pittmann, 2005), a percentage that is much higher than non-military gender rates in nursing.

Although the military was willing to amend its policies, colleges and universities were not as accommodating, at least initially. Luther Christman (1988), one of the most prominent and controversial figures in nursing reform, described his experience attempting to pursue a nursing degree at the University of Pennsylvania. He was told by the female nursing administrators at the university that he would receive an "A" for all non-nursing courses in which he was enrolled but all "F's" in his nursing courses simply because he was a man. Christman was even told that the University of Pennsylvania 
would never graduate a male nurse. Christman was also denied entrance into the Army Nurse Corps during World War II. He studied psychiatric nursing at the Institute of Pennsylvania School for Men before transferring to the Methodist Hospital nursing school in Philadelphia for financial reasons. In 1965, Christman completed his Ph.D. in nursing at Michigan State University. He became the first male dean of a nursing school in the United States in 1967 at Vanderbilt University. He later transferred to the Rush University College of Nursing in Chicago where he served as vice president for nursing affairs. Although Christman managed to overcome gender discrimination and become an educated nurse, such biases persisted in nursing until males accounted for less than $1 \%$ of the nursing workforce during the 1960s (Kalisch \& Kalisch, 1978), a precipitous decline from the $13 \%$ males constituted in the 1890 s (O'Lynn \& Tranbarger, 2007). Due to an increase in associate-level nursing programs and amended higher education practices, the percentage of males in the nursing workforce increased to $2.7 \%$ in the $1970 \mathrm{~s}$. This amount has continued to rise to the current $9.6 \%$ of registered nurses being male (U.S. Census Bureau, 2013). Despite this positive trend, nursing continues to be a femaledominated profession posing numerous barriers for men that hinder their academic retention rates and limit their career advancement.

\section{Barriers Facing Men in Nursing}

Some scholars argue that insufficient efforts have been made to recruit men into the nursing profession in recent years (Meadus \& Twomey, 2011; Wolfenden, 2011), while others contend that academic programs are making advances in the recruitment of men (McLaughlin, Muldoon, \& Moutray, 2010; Williams, 2002). The current percentage 
of male registered nurses $(9.6 \%)$ is roughly twice what it was in 1992 (American Association of Colleges of Nursing, 2006; Bureau of Labor Statistics, 2012; U.S. Census Bureau, 2013). Although there has been a steady increase in the number of male nurses in recent years, they still constitute relatively few of the over 2.6 million registered nurses currently in the workforce. One of the contributors to the low male representation is that men face barriers to pursuing a nursing career that women do not. These barriers include the lack of male academic role models, a significantly reduced male presence in the nursing workforce, gender-biased language, and occupational stereotypes. Men commonly encounter these barriers prior to applying to nursing programs, during nursing-related classes, and in the clinical setting. As one anonymous male nurse stated in Hart's (2005) manuscript addressing the stereotypes facing male nurses, "we are not too stupid to go to med school, we're not all gay, we're not in it to meet women, we're not lazy. We can be trusted" (p. 48). While men in nursing do face unique barriers, a study by Laroche and Livneh (1983) investigated predictors of attitudes toward male nurses. Their findings indicated that educational level and sex serve as significant predictors. Specifically, as one's educational level increases, so does their acceptance of male nurses. Additionally, the authors noted that women are more accepting of male nurses than are men. The coming sections will look to specifically address the experiences of male nurses by focusing on their experiences in academics with specific emphases placed on gender-based barriers and stereotypes.

Experiences of male nursing students. Motivated by the current shortage of registered nurses paired with the limited presence of men in the field, nursing programs are actively seeking growth in program offerings and student enrollment - which includes 
differing degrees of dedication to the recruitment of men. Although scholars vary in their assessments of the efforts being put forth to generate more male nursing students, a report by the National League for Nursing (2007) tracked male enrollment in nursing programs between 1985 and 2006. In 1985, approximately $6.3 \%$ of nursing students were men. This percentage rose to around $8.2 \%$ in 1989 and then to a high of $13.3 \%$ in 1995 . Male enrollment then dipped in 2003 to $9.8 \%$ prior to rising to $12.1 \%$ in 2006 (American Association of Colleges of Nursing, 2006). Increases in male enrollment have been particularly notable at nursing programs at universities in Iowa, Washington, Pennsylvania, and Texas.

At the University of Iowa, the College of Nursing formed a "Men in Nursing" mentoring program. The goal of this program is to "encourage men of all ages to become nurses and join together with all nurses in strengthening and humanizing health care" (University of Iowa, College of Nursing, 2012). The University of Washington has a dedicated webpage offered through the School of Nursing that discusses the resources available to male nurses "as a means of enhancing an environment that welcomes men in nursing" (University of Washington, School of Nursing, 2012). In the School of Nursing Science at the University of Pennsylvania, a male nursing group was created under the acronym "MAN-UP," which stands for Male Association of Nursing at the University of Pennsylvania. One of the main objectives of this organization is to "provide a forum for discussion of factors affecting male nurses and men interested in pursuing a career in nursing" (University of Pennsylvania, School of Nursing, 2012). Finally, at the University of Texas at Austin, students formed the "Longhorn Association of Men in Nursing," which looks to advance the social acceptability of nursing as a career path for 
males (University of Texas at Austin, School of Nursing, 2012). In a review of nursing school recruitment materials, O'Lynn and Tranbarger (2007) noted that programs are making more of an effort to appeal to male audiences by presenting nursing in a genderneutral light. The effort of these programs, and others like them, have contributed to the increased male population currently in nursing schools across the nation. However, despite this positive trend in recruitment efforts, research still points to male nursing students as being at a disadvantage when compared to female students.

Much of the research on mens' underrepresentation in nursing has focused on nursing program attrition rates, as indicated by the percentage of students who enter a program minus those who persist in enrollment to complete it (Gallie, 2005). Male attrition rates in nursing classes far exceed those of their female counterparts (Mulholland et al., 2008) with researchers suggesting that between $40-50 \%$ of males who enroll in nursing classes either drop, fail the course, or transfer to a non-nursing class (Poliafico, 1998; Stott, 2007; Villenevue, 1994; Wilson, 2005). In other words, they do not finish the class or program that they started. This contrasts with the attrition of female nursing students, which is 17-25\% (Finlayson et al., 2000; Godfrey, 2000; Lipley, 2000). To put these statistics in reference of the overall gender-based attrition rates across all majors on college campuses (not just in nursing programs), female student attrition rates are approximately $17-18 \%$ and male students range between 22-31\% (Severines \& Dam, 2012). Thus male attrition rates are significantly higher in nursing than the standard across campus, while female attrition rates stay constant. Commonly cited reasons for female attrition rates include work and family responsibilities, financial issues, and career change (Brady \& Sherrod, 2003; Mulholland et al., 2008). Male nursing students not 
only deal with these same three factors, but are also faced with additional stressors that female students do not face within nursing education. The two most frequently cited stressors related to male nursing student attrition rates are gender-based barriers and gender stereotypes (O’Lynn, 2004). These barriers and stereotypes influence how men are perceived in the nursing classroom, which contributes to their difficulties in nursing programs. Both gender-based barriers and gender stereotypes will be discussed in greater detail below as they relate to the experiences of the male nursing student.

Gender-based barriers in nursing school. In a study of barriers facing men in nursing programs, O'Lynn (2004) developed an Inventory of Male Friendliness in Nursing Programs (IMFNP). The IMFNP identified specific barriers that male nursing students face based solely on their gender, which have the potential to lead to difficulties in the nursing classroom. Three of the most prevalently cited barriers by the study's sample were: (1) lack of male mentors, faculty, and clinical instructors, (2) the overall lack of a male presence in the classroom, and (3) gender-biased language within the classroom and course materials. In a summary of his study's findings, O’Lynn (2004) stated that nursing programs are not geared towards overcoming these barriers and therefore are unable to attract, retain, and effectively train men for the nursing workforce. More recent research has sought to further explicate each of these distinctive barriers to provide a greater understanding of the male experience within the nursing curriculum (Gilchrist \& Rector, 2007; Meadus \& Twomey, 2007; O’Lynn, 2004; O’Lynn \& Tranbarger, 2007; Tracey \& Nicholl, 2007).

As detailed by Meadus and Twomey (2007) as well as O'Lynn (2004), the lack of male mentors and role models as well as limited male faculty and clinical instructors - 
thus an overall lack of a male presence - is the most significant contributor to the hardships and attrition rates of male nursing students. As it currently stands, approximately $3.5 \%$ of nursing faculty are men (O’Lynn \& Tranbarger, 2007). Due to the guidance and support offered in student-instructor dyads, this overall lack of male mentors, instructors, and faculty in nursing programs has become a point of contention for male nursing students. The most frequently cited and well-respected endeavor investigating the student-instructor dyad in nursing is that of Bell-Scriber (2008), who asked male and female nursing students to evaluate their interpersonal experiences with their nursing instructors. Women most frequently cited feeling support from their instructors, a stark difference when compared to their male counterparts who expressed feeling an overall lack of support. In a study on perceptions of educational experience by male nursing students, Paterson et al. (1996) noted that not only did male students not feel the same degree of support as did women, but they also felt more scrutinized and "under the microscope" (p. 34). The gender minority status of male nursing students makes them more visible and in turn, makes it more difficult for them to blend in to their surroundings. Due to their experiences as male nurses, male nursing faculty contribute a unique perspective to the academic setting, which has the potential to increase male nursing student retention through discussions of shared experiences and mentoring on how to overcome barriers and stereotypes (Keogh \& O’Lynn, 2007).

In addition to the lack of male mentors, faculty, and clinical instructors, another frequently cited barrier for male nursing students is the overall lack of a male presence in the student body and curriculum. Kippenbrock (1990) examined 279 B.S.N. programs in the United States to determine the degree to which men were being recruited into the 
major. Of the programs included in the study, the student body was $5.3 \%$ male, with $12 \%$ of the schools having no men at all in their program. Today, men account for approximately $12.1 \%$ of nursing students (American Association of Colleges of Nursing, 2006; National League for Nursing, 2007). Within the previously cited Bell-Scriber (2008) study of nursing student-instructor interactions, female participants noted that every semester they noticed more male students leaving the program - leading to a reduced male presence in the classroom. These female students offered a potential rationale for this attrition rate as they felt faculty and other students unnecessarily discriminated against their male counterparts. In describing her experiences with male nursing students, a female instructor interviewed by Bell-Scriber (2008) stated:

I think a lot of the males that I experience that go into nursing, they're going into it for the wrong reasons. Whatever they wanted to do didn't work out. And they have more of a chip on their shoulder, a confrontational attitude. Like we have to prove ourselves or something (p. 147)

Following up with this same female instructor, she was asked if she would like to see more males recruited into her program. Her response was:

No, I'd like to get rid of the men. They all wanta be... anesthesiologists. The majority of males that I have taught clinically are not in nursing for the reasons I think they should be in nursing. They wanta be PAs or they want to be nurse anesthetists (p. 147)

This is not to say that one individual is outlining the standard set by all nursing educators. However, statements like these begin to shed light on why men are apprehensive about entering into or continuing nursing as a field of study - they are not experiencing the same supportive learning environment from female faculty. Although women are commonly encouraged to transition into traditionally male-dominated professions, men 
are not receiving the same societal encouragement to enter nursing (Boughn, 1994; MacPhail, 1996; Poliafico, 1998; Wilson, 2005). Combine this with the increased percentage of men who drop or transfer from nursing courses (Poliafic, 1998; Villeneuve, 1994; Wilson, 2005) and there is a major lack of a male presence in the collegiate nursing classroom.

As for nursing instruction, combinations of historical and linguistic factors serve to focus classroom treatments of the field's legacy and future on the contributions of its female practitioners. The language used in the nursing classroom serves as a form of discrimination against men in the field (Wolfenden, 2011). For example, have you ever used or heard the term female nurse used by another person? Most likely not. That female is simply a nurse. On the other hand, it is not uncommon for men to be labeled as male nurses (Meadus, 2000). As outlined by O'Lynn (2004), there is a societal assumption that nurses must be women and when they are not, it is necessary to qualify that individual as a male nurse instead of simply being labeled as a nurse. In other words, nursing has been sexualized as women's work (Villenevue, 1994). Further, Smith (2006) noted that course materials and classroom lectures frequently use female and nurse interchangeably. Not only is the language slanted towards the female student, but the history and contributions of men to nursing are also being omitted - these serve to further perpetuate the gender bias faced by men in nursing (Anthony, 2004). In an attempt to further assess gender-biases in nursing materials, Bell-Scriber (2008) evaluated three of the most popular nursing textbooks in press at the time of study. Her review noted that all illustrations, pictures, and scenarios involved only female nurses. In a different phase of the same Bell-Scriber (2008) study, a male participant stated that he was alarmed at the 
lack of male representation in nursing textbooks, ultimately making his role of being a gender minority more salient. Similar findings related to the gender-biased slant in the nursing curriculum have been replicated in other studies (e.g., Kelly, Shoemaker, \& Steele, 1996; O’Lynn, 2004; Smith, 2006). Slowly but surely, the face of nursing is beginning to shift; however, the educational materials along with course instructors are demonstrating a lack of progress by not representing nurses as both male and female, despite a call to action made in the early 1990s by Shellenbargen (1993).

In combination with a lack of male mentors, faculty, clinical instructors, and male presence in the classroom, the use of gender-biased language creates a barrier that makes it more difficult for men to succeed in the field of nursing. For the current study, male nursing students will be sampled as opposed to current professionals, which is a commonly used substitution strategy for research (see Harding, 2007). Based on the salience of attrition rates, gender-based barriers, and stereotypes (detailed in the following section), nursing students are prime candidates for the current investigation that tests the role of "stereotype threat" on nurse-patient interactions.

Gender stereotypes. Gender-based stereotypes typically fall on a continuum, with presence in one gender equating to absence in the other (e.g., weak - strong). In terms of gendered stereotypes, female attributes include being empathetic, caring, nurturing, communal, expressive, weak, passive, and emotional (Aries, 1996; Broverman et al., 1972; Flannery, 2000; Rosenkrantz et al., 1968; Spence, Helmreich, \& Stapp, 1974; Williams \& Best, 1990). In contrast, men are stereotypically portrayed as authoritative, agentic, competent, rational, strong, aggressive, cold, and emotionless (Aries, 1996; Broverman et al., 1972; Eagly \& Steffen, 1984; Flannery, 2000; Spence et al., 1974). 
These stereotyped attributes have held consistent over time, with current investigations replicating those from the mid-1900s (Bergen \& Williams, 1991; Fecteau, Jackson, \& Dindia, 1992; Flannery, 2000). Further exploring this distinction between gender stereotypes, some researchers have found that male attributes and characteristics are perceived as more desirable than those of women (Bakan, 1966; Broverman et al., 1972; Dennis \& Kunkel, 2004), while others have argued that there is no inherent difference (Rudman \& Kilianski, 2000; Williams \& Best, 1990). Despite this lack of consistency among researchers in identifying the most socially desirable male and female attributes, researchers have found consistency in noting that gender stereotypes are influential in what occupations are acceptable for men and women to hold.

To further the academic endeavor into understanding which occupations are gender- typecast, Shinar (1975) conducted a study to determine if gender stereotypes influence occupational choices, and if so, which positions are stereotyped as male or female. To test her research questions and identify occupations that are typecast based on gender stereotypes, she looked at the proportion of men and women currently working in specific professions. This investigative method aligns with previous (e.g., O’Dowd \& Beardslee, 1967; Oppenheimer, 1968) as well as more current research (e.g., Beggs \& Doolittle, 1993; Sastre, Fouquereau, Igier, Salvatore, \& Mullet, 2000). Results from Shinar's (1975) study indicated that registered nurse was the second most feminine occupation next to manicurist. As reported by Kauppinen-Toropainen and Lammi (1993), female-gendered occupations focus on taking care of others, while male-centered occupations are on a more supervisory level. Furthermore, researchers have stressed that gender stereotyping is more salient in female occupations (Deaux, 1995). As noted by 
Egeland and Brown (1988), these barriers elicit a role strain that can deter men from considering nursing as a viable occupational option. With men accounting for approximately of $12.1 \%$ and $9.6 \%$ of nursing students and registered nurses respectively (American Association of Colleges of Nursing, 2006; National League for Nursing, 2007; U.S. Census Bureau, 2013), nursing meets the previously established criteria of being identified a "gendered occupation."

Gender issues associated with nursing. The field of nursing experienced a significant shift over 150 years ago with the introduction of the Nightingale Training School for Nursing. Based on the work of Nightingale and others like her, nursing has become a stereotypical female profession with the contributions of men to the field being frequently overlooked. To Nightingale, women were a natural fit for nursing due to their capacities to be caring and empathic. Although society has clung onto this perception of what a nurse should be, Lo and Brown (1999) argue, "intelligent nurses do not belong to any specific gender, and both female and male nurses may be educated to deliver a professional nursing service" (p. 41). From a social standpoint, it is completely acceptable for a woman to be a nurse as they are expected to be caring, nurturing, and sensitive to the needs of others (Anker, 1998; Heilman, 2001; Shinar, 1975). Women are expected to be more helpful and giving in the workplace, and nursing is one of those occupations where these attributes are exalted. On the other hand, male attributes of authority and assertiveness are advantageous in the corporate world (Eagly \& Steffen, 1984). It is safe to say that these societal perceptions of gendered stereotypes are a significant contributor to the reduced amount of male nurses and nursing students in this country. As stated by Deaux (1995), nursing emphasizes nurturing and helping qualities. 
Based on findings from the previously cited research along with popular opinion, nursing is a female-typed occupation. Therefore, the remainder of this section will address stereotypes faced by men that choose to pursue nursing as a profession.

For men who decide to enter nursing, there is an increased risk of failing (or perceived to be at a deficit) due to stereotypical barriers encountered by being a gendered minority (Pryhmachuk, Easton, \& Littlewood, 2008). The stereotypes faced make it difficult for men to stay active in the field over an extended period of time - within four years of graduating, $7.5 \%$ of men quit nursing compared to $4.1 \%$ of female nurses (Yurkovich, 2006). In 2005, a Bernard Hodes Group national study surveyed 490 male nurses about why men were reluctant to make nursing a career choice. Results from their investigation highlighted assumptions that nursing was a female profession, that men cannot be caring, and participants did not want to be labeled as homosexual. Research also notes that male nurses feel they are treated differently - not necessarily unfairly - by peers and supervisors based on their gender (Anthony, 2006; Cudé \& Winfrey, 2007). Citing to gender-biased stereotyping associated with nursing, male nurses tend to enter specialty areas where they can be more hands-off with patients such as anesthesia, psychiatry, and administration (Inoue et al., 2006; McRae, 2003; Squires, 1995) and that have higher technical and pressure components (Dassen et al., 1990; Lo \& Brown, 1999; McDougall, 1997; Streubert, 1994). This preference simply reinforces the perception that nursing is female's work.

Perhaps one of the most significant barriers keeping males from pursuing more hands-on nursing positions comes from stereotyped characteristics of how nurses behave and interact with patients. Of these expectations, one of the most fundamental and 
studied characteristics of nursing is caring (Brilowski \& Wendler, 2005; Godkin \& Godkin, 2004; Horrocks, Anderson, \& Salisbury, 2002; McDougall, 1997; Milligan, 2001; Scotto, 2003; Spichiger, Wallhagen, \& Benner, 2005), with caring being identified as the essence and unifying theme of nursing practice (Leininger, 1991; Morse et al., 1990). As defined by Leininger (1984), caring is:

The direct (or indirect) nurturant and skillful activities, processes, and decisions related to assisting people in such a manner that reflects behavioral attributes which are empathetic, supportive, compassionate, protective, succorant, educational, and others dependent upon the needs, problems, values, and goals of the individual or group being assisted ( $\mathrm{p}$. 4)

From this definition, it is important to highlight that caring is multifaceted and involves attributes such as empathy, support, and compassion - all attributes typically connected to women. In further developing the gender stereotyping of nurses and caring, caring comes about from the development of relationships between nurses and patients (McCance et al., 1999; Roach, 1984; Watson, 1985), with caring being "an interpersonal process that is characterized by expert nursing, interpersonal sensitivity, and intimate relationships" (Finfgeld-Connett, 2008, p. 198). Davies (1995) further supports this relational development by noting that caring involves a commitment to the patient physically, mentally, and emotionally. As such, the fundamental core concept of nursing is caring - one of the most commonly cited female attributes. Therefore, it is this expectation that nurses will demonstrate care, nurturance, and other feminine attributes rather than masculine attributes that pushes men away as well as making it difficult for them to be accepted by patients. White and White (2006) label this relationship a congruent pair-- that is, female and nurse are completely acceptable under the social 
microscope. On the other hand, male and nurse are incongruent, therefore dissuading men from pursuing nursing. This social equation in no way means that men cannot be caring and provide quality nursing care (Ekstrom, 1999), but those men who demonstrate caring are in the social minority, as the caring role is dominated by women (McDougall, 1997, p. 811).

In addition to the stereotype that men are less caring than women, an additional stereotype facing prospective male nurses is that they must be homosexual to want to be a nurse (Armstrong \& Fiona, 2002). Gender-based stereotypes surrounding nursing imply that a quality nurse is one who is caring, nurturing, and emotional - all attributes not typically associated with the masculine, heterosexual self-identity. As noted by McDougall (1997), “Of course not all men will follow traditional masculine paths however, it appears that the majority will. One group of men that perhaps follow a different path are gay men. Gay men in nursing have become more prominent..." (p. 812). Whether homosexual or not, men wishing to enter the female-dominated field of nursing are often met with the social perception that they must be gay because they are nurses (Loughrey, 2008). This expectation creates a gender-role conflict (O’Neil, 1981a, 1981b) in a significant proportion of men, which prompts them to either not pursue nursing or to actively demonstrate their heterosexuality (Lupton, 2006). For men who hold tightly to traditional views of masculinity, this gender-role conflict creates a tension due to perceived public perceptions of potential homosexuality. As noted by Harding (2007), "gay men are not considered 'real' men and...men who are nurses are reframed as gay" (p. 637). Research focusing on hegemonic masculinity - a socially defined set of rules for what it means to be man - explores how men carry out their personal 
relationships and identities with an emphasis on being in opposition of others (Brannon, 1976; Connell, 1995; Kimmel, 1997). Donaldson (1993) argues heterosexuality and homophobia are at the core of hegemonic masculinity. Perceptions and worldviews such as these are contributing to the decreased roles of men in becoming nurses. In terms of measuring the masculinity of men in female-dominated professions (e.g,. nursing, elementary school teachers), Galbraith (1992) conducted a study using the Bem Sex Role Inventory (Bem, 1974). He found that a majority of male participants who were nurses identified themselves as androgynous (scoring highly on both masculine and feminine measures), with less than a quarter of male nurses identifying themselves as masculine. With such a small percentage of male nurses seeing themselves as masculine, the gendered stereotype of nursing not being an acceptable position for males is further reinforced.

The bottom line is that not all jobs are distributed equally between men and women, and nursing has one of the most lopsided inequality ratios of all professions. The two previously outlined gender-based nursing stereotypes associated with caring and perceived homosexuality serve as deterrents that prevent many interested men from entering nursing. The inability of men to overlook such stereotypes only contributes to the lopsided gender distribution in the nursing workforce (Alpert \& Breen, 1989; Miller \& Budd, 1999; Miller, Neathey, Pollard, \& Hill, 2004; Nelson \& Belcher, 2006). For those that do go on to enter nursing, significant percentages actively select positions that are more hands-off with patients such as anesthesia, psychiatry, and administration (Evans, 1997; Squires, 1995). These specialties hold the capacity to reduce the amount of gender-role conflict felt due to limited patient interaction. This is not to say that all 
men who choose nursing are dissatisfied with their professions (Rochlen, Good, \& Carver, 2009), but there are different standards and expectations for men pursuing degrees and working in a female-dominated profession. The following section will look to compare the experiences of male nurses with the experiences of men in other female-dominated professions.

Gender issues associated with other female-dominated professions. The question of how people make nontraditional career choices has garnered a significant amount of attention over the past 15 years (Anker, 1998; Bresnahan, Inoue, Liu, \& Nishida, 2001; Duehr \& Bono, 2006; Ji, Lapan, \& Tate, 2004; Rochlen et al., 2009). In today's society, women are frequently encouraged to extend themselves by seeking nontraditional professions (e.g., physicians). Although there is growing public acceptance for women pursuing many traditionally male-dominated professions, there is less acceptance of men in traditionally female-dominated professions, which in turn explains why relatively few men choose to enter these fields. Bradley (1993) suggests that men consider entering a female-dominated profession when one of three criteria is met: (1) the position in question has recently had an increase in the use of technology, which would allow the men to demonstrate their "tech-savviness," (2) there has been an elimination of occupationally-based gender roles, or (3) there is an overall lack of other employment opportunities. One of the main reasons men do not seek out stereotypical female professions outside of the aforementioned three is that men would have to be willing to accept less salary and status (at least to start). Lupton (2006) notes that very few men actively choose to enter into female-dominated professions, with men from working-class backgrounds entering them out of financial necessity. Men from middle-class 
backgrounds, on the other hand, are raised to enter male-dominated professions where there is prestige and higher pay.

When a profession is deemed "natural" for one gender (e.g., women in nursing), this same profession is often times rendered as "unnatural" for the other (e.g., men in nursing). Occupations, such as nursing, have become female-dominated as: 1) they are seen to require a more feminine-based skill set, and 2) there are more women than men employed in said occupation (Williams \& Villemez, 1993). In addition to this dissertation's focus on nursing as a female-dominated profession, other fields like education, social work, and childcare are commonly typecast as female as well. Expectations regarding work duties of these professions are comparable to the role of the woman in the household - the caretaker and nurturer. As noted by Cameron and Moss (1998), "caring work is gendered not just because the workforce is nearly always women but because of the way the work is thought about" (p. ix). In contrast, male-dominated professions are those that require technical expertise or physical strength. Based on such, professions such as engineering, law enforcement, and construction are predominately thought of as male. In a longitudinal study of male entry into female-dominated professions in the U.S., Jacobs (1993) suggested that such employment is brief and operates on a revolving door paradigm where men who enter into female-dominated professions are quickly returned to male-dominated work.

In relation to gender-barriers experienced by men in other female-dominated professions, the most commonly cited barriers are threats to masculinity. As stated by Cockburn (1988), men who enter female professions are labeled as effeminate and looked upon as a failure. Additional research has investigated the influence of the masculine 
identity on non-traditional occupational choices (Chusmir, 1990; Jome \& Toker, 1998). Findings from these studies suggest that men employed in female-dominated professions do not endorse traditional masculine values and present a significantly less masculine self when compared with men in male-dominated professions. For those men in femaledominated professions out of necessity (e.g., financial), they can be pushed to actively promote their masculinity in the workplace and/or refrain from disclosing their profession to others out of fear from encountering prejudiced responses (Connell, 2000; Williams, 1995). These pressures to actively exert one's masculinity or hide professional disclosure can push men to be ashamed of their professions, which influences individual levels of job satisfaction and burnout. This research into gender issues associated with femaledominated careers further highlights the biases experienced by men in predominantly female professions -biases that are comparable to those experienced by men in nursing. While token-status has the potential to hinder one's performance and satisfaction, working in a non-traditional field is not all bad as it does hold some benefits (i.e., higher pay, faster promotions). Building on the previously cited research on the biases and stereotypes of men in female-dominated professions, the next section of this dissertation highlights selected stereotype research that has been highly influential to the study of stereotype threat.

\section{Research Influencing Stereotype Threat}

Stereotypes have been a focus of social scientific research since the early 1920 s (Gilbert, 1951; Karlins, Coffman, \& Walters, 1969; Katz \& Braly, 1933; Lippmann, 1922). At the forefront of this research was journalist Walter Lippmann (1922) who 
borrowed the term stereotype from his experience in printing and applied the term to the social sciences in his book Public Opinion. To Lippmann (1922):

The attempt to see all things freshly and in detail, rather than as types and generalities, is exhausting, and among busy affairs practically out of the question...Instead we notice a trait which marks a well known type, and fill in the rest of the picture by means of the stereotypes we carry about in our heads (pp. 88-89)

In other words, stereotypes derive from an attempt to organize and comprehend the world around us. Following Lippmann, many social scientists have suggested that stereotypes are employed when we meet strangers, thereby interpreting their attributes in terms of common (and frequently erroneous) generalizations based on their gender, race/ethnicity, socioeconomic status, and other demographic factors. Stereotyping is not limited to these factors as individuals are commonly stereotyped based on their occupation - as will be further addressed in this dissertation. Dependent upon one's cultural upbringing, experiences, language use, social and cognitive development, stereotypes will be used differently (Miller, 1982). The different uses of stereotypes, as well as their shifting contents, was famously demonstrated in a longitudinal set of studies known as the "Princeton Trilogy" (Gilbert, 1951; Katz \& Braly, 1933; Karlins et al., 1969). In these studies, three generations of Princeton students were asked to select adjectives (lazy, ignorant, etc.) and rank order how well they felt the selected adjectives represented different ethnic groups (Katz and Braly, 1933). For example, $75 \%$ of participants in the Katz and Braly (1933) study identified African-Americans as lazy. To round out the Princeton Trilogy, Gilbert (1951) and Karlins et al. (1969) replicated this initial investigation by Katz and Braly (1933) to assess whether the content and consensus of the stereotypes changed over time. Findings from Gilbert's (1951) study noted that $31 \%$ 
of participants identified African-Americans as lazy, and only $26 \%$ of Karlins et al.'s (1969) sample made this same link between African-Americans and laziness. These results from the Princeton Trilogy highlight that over time, negative stereotypes appear to have the capacity to fade.

Despite what seems to be a positive finding, scholars have been quick to challenge the adjective-checklist methodology used in the Princeton Trilogy (e.g., Devine \& Elliot, 1995; Dovidio \& Gaertner, 1986). In fact, Dovidio and Gaertner (1986) as well as Devine and Elliot (1995) argue that the perceptions of African-Americans had not changed as drastically as the percentages from the Trilogy indicated, but that the adjectives used to describe these groups had changed. Therefore, the unchanged adjective checklist can potentially be partly accountable for the demonstrated findings. Regardless of methodological shortcomings, the findings nevertheless demonstrate the connection between stereotyping and social perception (Schneider, 2004). This demonstration strongly coheres with Lippmann's (1953) observation that stereotype ideation precedes person perception, not vice-versa: “...for the most part we do not first see and then define, we define first and then see... Inevitably our opinions cover a bigger space, a longer reach of time, a greater number of things, than we can directly observe" (pp. 61-62). Although Lippmann's conceptualization of stereotyping has also been met with criticism (Beeman, 2001; Perkins, 1979) it has brought to light the importance of understanding the interplay between stereotypes and our social interactions.

Perhaps the most influential study of stereotyping in the social sciences is Gordon Allport's (1954) The Nature of Prejudice. To Allport (1954), “The human mind must think with the aid of categories. Once formed, categories are the basis for normal 
prejudgment. We cannot possibly avoid this process. Orderly living depends on it" (p. 20). This frequently cited quote by Allport (1954) suggests that not only are biases and prejudices normal, but they are also culturally universal. Further elaborating on his ideas, Allport (1954) emphasizes stereotypes as a way of rationalizing one's prejudices, attitudes, and beliefs. Although this argument provides great insight into the role of stereotyping in social interaction, perhaps the most influential piece of Allport's (1954) book was his examination of the targets of prejudice and negative stereotypes. Allport (1954) theorized that a possible effect of belonging to a stereotyped minority group ("target") is the potential for unintentional confirmation of the stereotypes belonging to the "target" group. If one is aware of belonging to a stereotyped group, Allport (1954) noted that members of that targeted group hold the potential to internalize the stereotype, which in turn creates situational pressure, in effect causing the target group member to act in a way that is consistent with the negative stereotype already facing the group. Katz and colleagues (Katz \& Benjamin, 1960; Katz \& Cohen, 1962; Katz \& Greenbaum, 1963; Katz, Roberts, \& Robinson, 1965) empirically investigated Allport's (1954) claims by measuring the effects anxiety, threat, and racial composition have on academic performance of stereotype-targeted minority groups. Citing research by Dreger and Miller (1960) that African-Americans performed more poorly on tests than their White counterparts, Katz and colleagues set out to control racial factors to determine if they have an influence on testing scores. In two separate studies (Katz \& Benjamin, 1960; Katz \& Cohen, 1962), dyads were created consisting of African-American college students paired with either another African-American student or a White student to cooperatively solve problems. Results indicated that when placed with a White student, 
the African-American students performed at a lower level than when in same-race dyads. Katz and colleagues had no difficulties in attributing this decrease in performance to being placed in a "threatening situation," but they did experience issues pinpointing how the threat adversely affected performance. Specifically, two explanations were offered: 1) the African-American participants suppressed correct responses so that the White participants could retain their self-esteem and in turn would not be hostile towards the African-American participants for showing them up intellectually, and/or 2) the AfricanAmerican participants suppressed their interest so they would not have to be unfavorably compared to the White participants (Katz \& Benjamin, 1960; Katz \& Cohen, 1962). Despite their inability to offer a specific explanation as to how it occurs, the authors did bring to light the concept of "threat" having the ability to affect individual levels of performance. Specifically, being placed in a bi-racial dyad prompted increased levels of anxiety for the African-American participants, which in turn appeared to impair their problem solving performance.

In later studies, Katz and colleagues demonstrated that African Americans' intellectual performance suffered not only in the context of biracial interaction, but also when they believed a comparison between the two ethnic groups would be made. Katz, Epps, and Axelson (1964) presented African-American students with an IQ test and told them their results would be compared to either other African-American students or to White students. Those who thought their scores would be compared to other members of their ethnicity scored significantly better than their counterparts who expected a comparison with White participants. In addition, Katz, Roberts, and Robinson (1965) gave African-American students a similar intellectual performance test with half of the 
sample being told the test measured eye-hand coordination and the other half being told it would assess their intelligence. Framing the test as an eye-hand coordination exercise yielded significantly higher scores than intelligence framing. Both of these studies support Allport's claims about the plight of stereotype targets, and also led the way for the "stereotype threat" research paradigm that emerged in the mid-1990s.

\section{Stereotype Threat}

Over the last several decades, numerous studies have demonstrated that certain minorities (African-Americans, Latinos, and Native Americans) and women consistently score lower than White men on standardized tests (e.g., SAT, ACT, GRE, GMAT, etc.) and are underrepresented in STEM (science, technology, engineering, and math) fields (Betz \& Hackett, 1983; Fouad \& Smith, 1996; Gainor \& Lent, 1998). Different schools of thought have attempted to explain these performance gaps in terms of biological factors including genetics (Scarr \& Carter-Satzman, 1982; Thomas, 1993), differences in brain development (Benbow \& Stanley, 1980; Kimura, 1992), and evolution (Geary, 1996), while others have pointed to socialization as the cause for these differences (Eccles, 1987; Heller \& Ziegler, 1996; Ryckman \& Peckham, 1987). In contrast to these propositions, stereotype threat researchers have focused on situational factors as a potential explanation for these performance gaps (Steele \& Aronson, 1995). Specifically, they posit that a situationally salient, self-relevant stereotype can cause the target to be anxious, self-conscious, and distracted, which in turn can impair their performance on an intellectual task (Steele, 1997). 
In their seminal work, Steele and Aronson (1995) coined the term "stereotype threat" as a means of explaining the psychological discomfort that comes from the fear of confirming a negative stereotype about one's group. When a stereotyped individual is placed in a threatening situation, in an attempt to disconfirm the active stereotype, the individual experiences a performance deficit that ultimately confirms the stereotype (Spencer, Steele, \& Quinn, 1999; Steele, 1997; Steele \& Aronson, 1995; Steele, Spencer, \& Aronson, 2002). As outlined by Steele, Spencer, and Aronson (2002), stereotype threat occurs:

When a negative stereotype about a group that one is part of becomes personally relevant, usually as an interpretation of one's behavior or an experience one is having, stereotype threat is the resulting sense that one can then be judged or treated in terms of the stereotype or that one might do something that would inadvertently confirm it (p. 389)

Take for example the following quote from Dr. Neil de Grasse Tyson (1991), the $7^{\text {th }}$ African-American astrophysicist in the United States, doctoral graduate from Columbia University, and director of the Hayden Planetarium in New York City:

... in the perception of society my athletic talents are genetic; I am likely a mugger or a thief; my academic failures are expected; and my academic successes are attributed to others... to spend most of my life fighting these attitudes levies an emotional tax that is a form of intellectual emasculation

This emotional tax and intellectual emasculation that Tyson speaks of is a form of stereotype threat. Later on in the same address, Tyson stated that he is surprised that he made it as far as he had, to become the $7^{\text {th }}$ African-American astrophysicist (there are over 4,000 astrophysicists in the United States). This apprehension felt from being judged negatively due to his minority status influenced Dr. Tyson's expectations and abilities to perform. Not only does stereotype threat research concentrate on academic 
performance, it has also focused on intellectual disengagement (Major, Spencer, Schmader, Wolfe, \& Crocker, 1998), avoidance (Davies, Spencer, \& Steele, 2005), and disidentification (Crocker, Major, \& Steele, 1998). Since Steele and Aronson's (1995) seminal work, and especially within the past decade, stereotype threat has become one of the most commonly studied social psychological concepts (Schmader, 2007).

Steele and Aronson's (1995) seminal piece was the first to investigate the impact of stereotype threat on minority academic performance in a series of four studies. Previous work had demonstrated that when placed in a situation (e.g., competition, comparison, audience approval) where performing well is of utmost importance, or when a person is a token group member, that person's performance often suffered (Baumeister, 1984; Katz et al., 1965; Lord \& Saenz, 1985; Lord, Saenz, \& Godfrey, 1987). Steele and Aronson (1995) sought to determine whether a situation in which a negative self-relevant stereotype was salient also impaired performance. In their first experiment, Steele and Aronson (1995) recruited African-American and White undergraduate students from Stanford and asked them to complete an edited version of the GRE verbal section. Before taking the test, students were placed in either a "diagnostic" condition hypothesized to induce stereotype threat or a "non-diagnostic" control condition. In the diagnostic condition, participants were told the test was indicative of intellectual ability. The goal with this condition was to activate the negative cultural stereotype regarding African-Americans' intelligence. In the non-diagnostic control condition, students were simply told the test was a laboratory problem-solving task and was not indicative of intellectual ability. In theory, the negative stereotype would not be activated for participants in the non-diagnostic condition, which is opposite of the predicted 
relationship for the diagnostic condition. Controlling for previous standardized test scores (e.g., SAT), African-American participants performed at a significantly worse level than their White counterparts in the diagnostic condition, and also scored worse than African-Americans in the non-diagnostic control condition, whose SAT-adjusted scores did not significantly differ from Whites in this condition. Results from their second experiment replicated those of the first, but also observed that African-American participants in the diagnostic condition attempted fewer test questions than their White counterparts or African-Americans in the non-diagnostic condition. This points to conditions of threat not only hindering one's level of performance, but also their rate of question completion.

In their third experiment, Steele and Aronson (1995) assessed whether the performance deficits of African-Americans in the aforementioned studies were attributable to stereotype threat activation per se. As before, African-American and White participants were asked to complete an exercise framed as either diagnostic of an intellectual ability (in this case, "higher verbal reasoning") or non-diagnostic of this ability. In this timed exercise, participants were given a series of word fragments (e.g., $\mathrm{ST}_{--}$E) and were asked to fill in the blanks of the fragment to form an acceptable English word (STARE, STORE, STATE, etc.). One subset of the word fragments were designed so that they could be completed to form words related to the topic of race ${ }_{-}{ }_{-} \mathrm{C}$ E could be completed as "RACE," _ _ A C K could be completed as "BLACK," etc.); a second subset could be completed to form words related to self-doubt (e.g., D U _could be completed as "DUMB," F L _ _ could be completed as FLUNK, etc.). These item subsets were included to determine whether the diagnostic condition directly cued 
concerns about the negative intelligence stereotype for African Americans - if it did, one would expect members of this group to be more likely to complete the target fragments with race- and doubt-related words. As was hypothesized, African-Americans in the diagnostic condition had significantly more race- and doubt-related completions than African-Americans in the non-diagnostic condition or White participants in either condition. When the test was presented as an intellectual assessment that was diagnostic of ability, African-Americans demonstrated greater cognitive activation of their own stereotypes and abilities as was demonstrated in the race- and doubt-related completions.

In addition to the race- and doubt-related activation measures, participants were asked to rate self-descriptors and preferences that are stereotypic of African-Americans and African-American culture (e.g., Rate the extent to which you enjoy rap music, basketball, etc.). Results indicated that African-Americans in the diagnostic condition were significantly more avoidant of conforming to African-American stereotypes than African-Americans in the non-diagnostic condition and the White participants in either condition. This finding points to being exposed to conditions of threat was enough for the African-American participants to attempt to distance themselves from racial stereotypes. Finally, participants were asked to: 1) identify how prepared they felt for the assessment (self-handicapping measure) and 2) identify their race on the demographic questionnaire. African-American participants in the diagnostic condition were more likely to make excuses for their performance (e.g., not enough sleep, inability to focus, felt the assessment was tricky or unfair) and were less likely to indicate their race on the demographic questionnaire. Only $25 \%$ of African-American participants in the diagnostic condition self-identified race, this is in contrast to $100 \%$ of participants in each 
of the other conditions self-identifying their race. The results from this third study clearly point to the diagnostic condition instructions causing African-American participants to experience apprehension, however, would these same results be observed if the assessment was not labeled as diagnostic of ability?

In their final experiment, Steele and Aronson assessed whether making one's race salient before completing a task would induce stereotype threat. Upon arrival to the testing site, participants were randomly assigned to a race-prime or no-race-prime condition. For participants in the race-prime condition, they filled out a short demographic questionnaire with the last question asking them to identify their race. Participants in the no-race-prime condition were not asked to identify race. The participants then completed the same verbal GRE assessment that was used in the first two studies. Additionally, the test was not presented as diagnostic of intellectual ability in either the race-prime or no-race-prime conditions. Results indicated that AfricanAmericans in the race-prime condition performed significantly worse than those in the no-race-prime condition. Despite the assessment not being presented as a measure of intellectual ability, having participants record their race prior to completing the test was enough to activate the negative stereotype of African-American intellectual inferiority. This in turn impaired the performance of those participants. As a collective, these four experiments come together to indicate the degree to which stereotype threat can have a negative impact on the intellectual performance of African-American students (minorities), even African-American students at a prestigious undergraduate institution who have previously demonstrated increased levels of academic performance. 
Since the publication of Steele and Aronson's (1995) seminal piece, numerous studies have demonstrated comparable stereotype threat effects on African-American intellectual performance (e.g., Blascovich, Spencer, Quinn, \& Steele, 2001; Hernstein \& Murray, 1994; Massey \& Fischer, 2005; McKay, Doverspike, Bowen-Hilton, \& Martin, 2002; Steele, 1997; Thernstrom \& Thernstrom, 2003). In these studies, AfricanAmerican participants placed in stereotype threat conditions performed at significantly lower levels relative to African-Americans in non-threat conditions as well as White participants in the same stereotype threat condition. This repeatedly documented performance gap can be attributed to making the negative intelligence stereotype salient prior to their completing the assessment.

Stereotype threat and women. Stereotype threat researchers have consistently demonstrated an interest in studying the academic performance differences of minorities (e.g., Steele, 1997; Steele \& Aronson, 1995; Steele, Spencer, \& Aronson, 2002). However, racial minorities have not been the sole focus of their research, as researchers have also dedicated time and resources to investigating gender performance differences. Of the gender groups studied, women have garnered the most attention regarding their mathematics performance as compared to men. The stereotype that women are inferior to men in math not only has the ability to curb a woman's interest in math as a subject (Jacobs \& Eccles, 1992), but also has the capacity to negatively impact their performance on math assessments (Brown \& Josephs, 1999; Nguyen \& Ryan, 2008; Schmader, 2002; Shih, Pittinsky, \& Ambady, 1999; Spencer et al., 1999).

Spencer et al. (1999) first demonstrated this impact in a three-part investigation. In their first experiment, male and female college students were asked to complete a 
quantitative subsection of the GRE examination. Test difficulty was varied, with half of the participants being given an "easy" test and the remaining half taking a "difficult" test. The "easy" test included standard math questions (e.g., algebra, geometry) while the "difficult" test assumed a working knowledge of calculus. To clarify, there were two separate tests, not simply different labels for the same assessment. Results from the study indicated that women performed at a significantly lower level than men when completing the difficult test, but scored comparably on the easier test. This findings supports earlier research demonstrating that in comparison to men, women underperform when taking difficult assessments but score similarly on easier ones.

In their second experiment, Spencer et al. (1999) asked men and women to complete the difficult version of the quantitative exam from Study 1. Half of each gender group was told the first section of the test had previously produced gender differences (threat condition) but the second section had not produced any gender differences (nonthreat condition). The remaining participants were given the same test, but given the opposite instructions - the first section had not produced any gender differences (nonthreat condition) but the second half had (threat condition). Participants were given 15 minutes to complete each section of the test. Results from the study indicated that women significantly underperformed in relation to men when completing the section they were told had previously produced gender effects. When completing the section that was stated to have produced no gender differences, no significant gender differences emerged. Thus by characterizing a portion of the test as sensitive to gender differences, the researchers induced a negative performance expectation that impaired female participants' performance. 
In a third experiment, participants were given a single math test and were told the test either had previously generated no gender differences (i.e., men and women performed comparably; control condition) or they were given no information regarding gender prior to their completion of the test (experimental condition). In the no-genderdifference (control) condition, there were no observed gender-based differences on performance. However, in the experimental condition, women underperformed in comparison to their male counterparts. Results indicate that when stereotype threat is actively reduced (i.e., men and women performed comparably), female performance increases. Being that women belong to a negatively stereotyped group regarding mathematics performance, making the stereotype salient or not reducing the stereotype, will negatively impact their performance. The findings from this investigation are significant to the progression of stereotype threat research for multiple reasons. First, Spencer et al. (1999) provide support for stereotype threat's influence on standardized test performance. Although prior work (e.g., Steele \& Aronson, 1995; Croizet \& Claire, 1998) investigated African-Americans and low socioeconomic students, this study stressed that any group is susceptible to stereotype threat if an assessment is framed in a manner that could make their group look bad if they perform poorly. Secondly, and quite possibly the most significant contribution of this study, is the focus on priming cue subtlety. As the authors progressed from Study 1 to Study 3, priming cues became less overt. In the final study, the threat condition did not contain an explicit threat prompt, yet still produced performance-based gender differences. The findings from the Spencer et al. (1999) studies draw attention to the impact subtle priming cues can have on participant performance. 
Extending beyond cue subtlety, additional scholars have investigated the performance of women as being domain-specific. Shih, Pittinsky, and Ambady (1999) investigated this exact issue using a homogenous Asian-American, female sample to complete a quantitative task. Prior to working on the assessment, participants were primed as being a woman (threat), Asian-American (non-threat), or receiving no priming in the control condition. Results from the study indicated that when primed as AsianAmerican (non-threat condition), women scored significantly higher on the mathematics assessment than the women-primed (threat condition) and control groups. There is a positive stereotype association between Asians and math, while there is a negative stereotype association between women and math, with these stereotypes playing out in this experiment. The authors identified this increase in performance for participants in the Asian-American prime as "stereotype lift." Based on these findings, Shih et al. (1999) conducted a second study, which was a cross-cultural examination to determine if performance was based on identity (i.e., Asian) or stereotypes (i.e., Asians are good at math). In their second study, participant recruitment occurred within a recently immigrated Asian community in Vancouver, Canada. Within this community, the stereotype that Asians are superior at math does not exist. Results indicated that activating the Asian stereotype (i.e., Asians are good at math) did not provide a performance lift to the participants as it did in their first study. Both the Asian-identitysalient (non-threat condition) and female-identity-salient (threat condition) conditions scored significantly worse than the control group. Therefore, without awareness of the positive stereotype (i.e., Asians are good at math), performance was not affected. These results indicate that stereotypes can both positively and negatively influence female 
performance on a math assessment without explicit instructions, and that social stereotypes are a significant determinant when multiple identities are involved.

McGlone and Aronson (2006) conducted a more recent investigation focusing on stereotype threat and potentially competing identities. In their study, they recruited men and women from a private liberal arts college in Northeastern United States. Participants were asked to complete the Vandenberg Mental Rotation Test (VMRT) as an assessment of visual-spatial reasoning - a domain in which women are stereotyped as inferior to men (McGillicuddy-De Lisi \& De Lisi, 2002). Prior to completing the VMRT, participants completed one of three questionnaires that made a specific social identity salient. The questionnaires primed for gender (e.g., List three reasons why one might prefer living on a coed floor in a dormitory), private college student status (e.g., List three reasons why one might attend a private liberal arts college), or as a resident of the Northeastern United States (e.g., List three reasons why one might prefer living in the Northeast to other parts of the US). As hypothesized by the researchers, women in the gender prime (threat) condition scored significantly worse than individuals in the private school identity (nonthreat) and Northeastern resident (control) conditions, with women in the private school (non-threat) prime achieving the highest female scores. As for the men in the study, those primed for gender achieved a higher score than the Northeastern resident group, while priming the participants' private college student status did not produce a significant lift from the control group - although the private college student primed group did score higher than the control group. As was demonstrated in the Shih et al. (1999) study, when multiple identities are involved, being primed as a positively stereotyped group member can lift one's performance while being primed as a negatively stereotyped group member 
can impair performance. Just as these previously cited studies have demonstrated that women perform significantly worse than men on math and spatial reasoning assessments under conditions of threat, the following section will address conditions that make men vulnerable to the pernicious effects of stereotype threat.

Stereotype threat and men. Although stereotype threat is most keenly experienced among groups historically targeted by negative stereotypes, it is a predicament that can beset anyone, because any group can be compared to another reputed to be at an advantage on some dimension (Aronson, 2002). Thus it can impair the performance of even those groups who are neither minority nor commonly stereotyped as having an ability deficit. White male university students, for example, bear no historical stigma associated with intelligence. However, in some circumstances comparisons with allegedly superior groups arise, thereby creating a situational threat. Aronson et al. (1999, Experiment 1) created such a threat when they gave White male undergraduates at Stanford University a challenging math test and told half of them their scores would be compared to those of Asian students. Mention of the intended comparison made students mindful of the stereotype of Asian mathematical superiority and consequently impaired their performance relative to others who were not told that this comparison would be made. The impairment occurred despite the fact that the White male students in the study had good reason to be confident in their math skills - most were math or engineering majors with SAT math scores over 700 .

In a second experiment, Aronson et al. (1999) replicated their initial findings but also investigated the role of math identification in the effect. The authors hypothesized that stereotype threat would be most disruptive for those who highly identified with math 
(Steele, 1997). White male undergraduates from the University of Texas at Austin who were enrolled in a stringent year-long calculus course (i.e., they were proficient in math) were recruited for the study. Prior to the experiment, the students completed a scale that rated their attitudes toward math, with students being classified as "high math-identified" or "moderately math-identified." As for the procedure, students were told that the upcoming test was to measure their mathematics ability and that their performance on the test would be compared with other students. These were all the instructions given to the control group, however, in the stereotype threat condition, participants were reminded that Asians were superior in math. Results supported the hypothesis in that the highlymath identified performed significantly worse in the threat condition than those in the control condition. For participants that were moderately-math identified, an opposite pattern emerged; they actually performed significantly better when in the threat condition. The authors posit that the degree to which an individual identifies with a domain can influence their susceptibility to stereotype threat. In this case, those who highly identified with math were more invested and looked to protect their ego by excelling on the test, whereas the moderately identified viewed the test as a challenge. Results from these two experiments indicate that it is possible for individuals in high-status groups (i.e., men) to be subject to the effects of stereotype threat. Additionally, these findings provide support for domain identification having an impact on stereotype threat outcomes. Dependent upon participant priming and identity activation in specific domains (i.e., male, non-Asian), stereotype threat can have a significant influence on participant performance.

Extending the study of stereotype threat outside the mathematical domain, Leyens et al. (2000) sought to assess gender differences in the processing of affective information. 
Stereotypically, women are considered better at recognizing and understanding affective information where men are often times viewed as incompetent (Bakan, 1966; Eagly, 1987). In their study, half of the participants were told the study was being administered to assess gender differences in the processing of affective information and that men generally perform worse than women in affective processing tasks (threat condition); the other half were told that the study was simply an exercise to help the researchers better understand the cognitive factors involved in verbal information processing (non-threat condition). After being randomly assigned to the threat or non-threat condition, students completed a lexical task where they had to identify prime words that popped up on a computer screen as affective, positive (valence), or if the presented word was actually a word (lexical). Results indicated that men in the threat condition were less accurate interpreting affective messages than those in the non-threat condition. As noted by Leyens et al. (2000), male participants in the threat condition actually overincluded affective words in an attempt to disconfirm the negative stereotype that men are not interpersonally sensitive and emotionally expressive. No differences were reported for the valence or lexical tasks. The findings from this experiment highlight that belonging to a low-status group is not a prerequisite to experiencing the effects of stereotype threat. In fact, the findings from this study emphasize that dominant groups (i.e., men) are susceptible to the effects of stereotype threat and that stereotype threat is not dependent upon an individual's current social status but that stereotype threat is situational.

When reminded of gender stereotypes characterizing them as less socially and emotionally sensitive in comparison to women, men have been found to be susceptible to the effects of stereotype threat (Briton \& Hall, 1995; Cozby, 1973; Thayer \& Johnson, 
2000). To further the study of men and stereotype threat in relation to social sensitivity, Koenig and Eagly (2005) investigated men's abilities to correctly interpret the expressive behaviors of others. Being that social sensitivity is often portrayed as a stereotypically female-oriented attribute, the authors hypothesized that men would perform significantly worse when placed in the social sensitivity diagnostic threat condition. For the study, men and women were assigned to a diagnostic social sensitivity threat condition or a nonthreat control condition. In the diagnostic threat condition, participants were told the test was a measure of social sensitivity, while participants in the non-threat control condition were told the test was a measure of information processing abilities. Additionally, in the threat condition, the experimenter told participants that men typically did not do as well on the assessment as women. To measure social sensitivity, the authors utilized Costanzo and Archer's (1993a, 1993b) Interpersonal Perception Task-15, which is a collection of short social interaction video clips (e.g., relationships, deception, status, competition). After each clip, participants were presented with a multiple-choice questionnaire that focused on their ability to accurately interpret the expressive behaviors of those in the video clips. Results indicated that men in the diagnostic threat condition were significantly less accurate in their interpretations than men in the non-threat conditions; female accuracy did not fluctuate between conditions. Additionally, men and women performed comparably in the non-threat condition.

In a post-test questionnaire, participants completed a 6-item "strategy" scale to determine if their responses were influenced by the use of intuitive (i.e., answering on the first impression) or deliberate (i.e., using principles of behavior to answer) processing strategies. Results indicated that the self-reported processing strategy used by 
participants served to moderate social sensitivity. In the social sensitivity threat condition when men self-reported as using a more deliberative strategy as opposed to intuitive, their performance decreased. This effect was not present in the non-threatening condition. The authors reported that as men became more deliberate in their strategy choice, they actually became more vulnerable to stereotype threat. Additionally, the authors suggested that the reported results derived from the impact stereotype threat had on the cognitive capacity for male participants. In other words, men using a deliberative processing strategy experienced a decrease in working memory capacity that was not observed in the men using automatic (intuitive) processing. In terms of expanding on the body of stereotype threat literature, this study provides support for stereotype threat being a resource-demanding phenomenon, and that men are susceptible to the effects of stereotype threat. For communication scholars, these findings suggest that stereotype threat can influence social interactions as well as one's ability to accurately interpret nonverbal cues.

The research up to this point demonstrates that it is not just women who are susceptible to the pernicious effects of stereotype threat - men are as well, and these effects can be demonstrated in multiple domains. Under conditions of threat, men underperformed in the domains of mathematics (Aronson et al., 1999), the processing of affective information (Leyens et al., 2000), and of social sensitivity (Koenig \& Eagly, 2005). In these studies, when there was no threat, no impairment in performance occurred. In terms of the current dissertation, these studies provide two distinct contributions: 1) they demonstrate that men are susceptible to the effects of stereotype threat, and 2) that domain-identification has the potential to influence the relationship 
between stereotype threat and performance. However, one question these studies do not address is if the effects of stereotype threat are present in non-testing conditions (i.e., timed quantitative assessments). In other words, can stereotype threat help clarify gender-based performance differences in the other evaluative contexts?

\section{Stereotype Threat and Communication}

Since Steele and Aronson (1995) introduced the theory of "stereotype threat," scholars have continued to investigate situational factors to offer explanations for assessment-based gender differences. For example, it is a well-known stereotype that female students are intellectually inferior to male students on mathematical assessments, and in fact, do perform worse than male students on standardized assessments. To address this gender difference, Quinn and Spencer (2001, Study 2) recruited high math achieving male and female undergraduate students to complete an 18-item assessment from the mathematical portion of the SAT. Prior to taking the assessment, half of the participants were told the assessment had previously demonstrated to be "gender fair" (reduced threat condition) while no mention of "gender fairness" was provided for the increased threat condition. In their study, men scored significantly higher in the increased threat condition - a situational factor that helps to explain this gender difference. For the reduced threat condition, while not statistically significant, female participants outperformed the men. Of interest in the current dissertation is if the explanatory value of stereotype threat is limited to the evaluative contexts of standardized testing. 
A significant focus of stereotype threat researchers has been concentrated in the standardized testing context, although researchers have claimed that the effects of stereotype threat can be observed in any evaluative context where behavior is evaluated (Aronson \& McGlone, 2009; Steele, 1997). What about contexts where a quantitative or spatial reasoning test score is not the evaluative outcome? For example, every student at the University of Texas at Austin is required to take Professional Communication, a public speaking course where they will deliver a handful of speeches to their classmates over the course of the semester. Additionally, as students transition out of UT and into the working world, they will most likely be interviewed for potential employment opportunities. In these situations, it is not a test score that is under evaluation, but the individual's communication skills that are being evaluated. Communication researchers have long recognized that when speaking in a context that is perceived as highly evaluative (e.g, speech class, interviews), communicators will experience anxiety (BoothButterfield \& Butterfield, 1986; Daly \& Buss, 1984; McCroskey, 1978, 1982). In a study controlling for the effects of state anxiety (how an individuals personality fluctuates dependent upon external stimuli), Greene and Sparks (1983) asked participants to complete a state anxiety scale in both an evaluative and non-evaluative context. Prior to completing the state anxiety scale, participants completed McCroskey's (1978) Personal Report of Communication Apprehension scale with participants being grouped in high and low apprehension groups. Greene and Sparks (1983) observed that in the nonevaluative context, participant state anxiety scores were comparable across the high and low apprehensive groups. However, in the evaluative context, highly apprehensive individuals were also more anxious when compared to the low communication 
apprehension group. Research by Patterson and Ritts (1997) demonstrated a correlation between communication anxiety and communicative performance, such as reticence to participate in communicative events and an overall increase in speech disfluency use. It appears, at least on the surface, that there is an overlap between the effects of stereotype threat in the testing domain and the effects of evaluation apprehension (Cottrell, 1972) on communication. One of the main guiding questions then, is stereotype threat useful in explaining gender-based performance differences in the evaluative context of communication when the communicator is faced with the potential of confirming a negative self-relevant stereotype?

As previously discussed, prior research has explained differences between men and women in quantitative, spatial-reasoning, and affective task performance. But what about communicatively? The main goal in this dissertation is to explore the potential effect of stereotype threat on communication, specifically within the nurse-patient relationship. With this research goal articulated, I now turn my attention to the limited body of stereotype threat research that has evaluated interpersonal interactions and communication, as these provide the foundation for the current investigation.

Prior works on social desirability (Goffman, 1963, 1973), the desire to project a positive self-image, have been cited as being highly influential in interpersonal interactions. This body of research suggests that individuals will put up a front to conceal their true feelings in order to be accepted by others. One area of focus for scholars has centered on the discussions of politics, especially in the interview context (Finkel, Guterbock, \& Borg, 1999; Silver, Anderson, \& Abramson, 1986). For example, a White interviewee, when being interviewed by an African-American, may say that he supports 
Barack Obama when in fact he voted for Mitt Romney because it may be seen as more socially acceptable in that dyadic context. Despite social desirability being potentially applicable to opinion-based and attitudinal surveys, it has come under scrutiny in relation to objective contexts. Davis and Silver (2003) addressed this issue by conducting telephone interviews about political knowledge and controlling for race-of-interviewer effects. Results indicated that African-Americans performed significantly worse, as evidenced by fewer correct responses, when interviewed by a perceived White individual (threat condition). The race of the interviewer was never disclosed and interviewees were asked to guess the race of the interviewer at the conclusion of the interview, which can lead to problematic conclusions in the findings. Despite this methodological pitfall, the Davis and Silver (2003) study does merit some attention as it utilized oral communication as a means of inducing conditions of threat in an objective context.

To eliminate the methodological issues of the Davis and Silver (2003) investigation, McGlone, Aronson, and Kobrynowicz (2006) substituted interviewer race for interviewer gender - a measure much more easily controlled for over the phone. Undergraduate students were contacted via telephone and asked questions used to measure their political knowledge. The authors hypothesized that female participants would be less accurate in their responses when placed under one of two conditions of threat: 1) when the woman was interviewed by a man, and 2) when the woman was told the questionnaire had previously produced gender differences. It was also hypothesized that men would achieve higher scores than women. The authors observed that women were significantly less accurate in their political responses when interviewed by men instead of women. Additionally, the female participants who were told the assessment 
had previously produced gender differences (diagnostic threat condition) were also less accurate in their responses. Female responses were comparable to the men when completing the assessment not under diagnostic conditions of threat. Male accuracy was not influenced by interviewer gender. In their discussion, McGlone et al. (2006) stated that when another woman interviewed women in the study or the interview questions were not posed as diagnostic of gender differences, the political knowledge gender gap closed. These findings reinforce the projections of Inzlicht and Ben-Zeev (2000) in that stereotype threat can be induced very subtly. In the case of these two studies, perceived interviewer race and interviewer gender served as the subtle inducing agent. This dissertation builds off the subtlety component that has been demonstrated in these two studies.

Although these two studies address stereotype threat in dyadic interactions, they were based on an objective assessment and did not specifically measure the gender-based communication patterns of their participants. Research into speech-based gender differences has commonly investigated the display of communicative patterns between men and women (e.g., Hannah \& Murachver, 1999; Hilpert, Kramer, \& Clark, 1975; Martin \& Craig, 1983; McMillan, Clifton, McGrath, \& Gale, 1977; Strodtbeck, 1951) as gender differences hold the capacity to place constraints on an individual's language use (Newman, Groom, Handelman, \& Pennebaker, 2008). One area of emphasis within this line of research is focused on the amount of words spoken during same- and mixed-sex interpersonal interactions (see Bales, 1950; Capella, 1985; Dovidio et al., 1988; Grob et al., 1997; Stein \& Heller, 1979) as verbosity has been positively linked to speaker dominance (Kleinke et al., 1973) and leadership qualities (Sorrentino \& Boutillier, 1975). 
An early investigation by Martin and Craig (1983) demonstrated that when two previously unacquainted individuals were placed in a room together and instructed to get to know one another, the men and women interacted differently. Specifically, when their partner was a man, both male and female participants used a similar amount of words. However, when talking to a woman, the amount of words uttered by male participants decreased and those for female participants increased. Perhaps the most commonly cited study of gender differences in relation to the amount of words spoken during interaction is that of Dovidio et al (1988), which investigated a different component in mixed-sex dyadic communication - the topic.

In their study, Dovidio et al. (1988) paired male and female undergraduate students who had previously had no contact with one another to complete a series of three short discussions. The topics of discussion were oil changing (masculine topic), sewing (feminine topic), and vegetable gardening (non-gender linked topic). Results indicated a significant effect for gender on both the masculine and feminine task topics. When discussing oil changing (masculine topic), men spoke more frequently than women, and on the feminine topic (sewing), women talked more often than did the men. For the nongendered topic (vegetable gardening), men spoke significantly more than did women. In outlining their findings, Dovidio et al. (1988) stated that when participants were discussing masculine or gender neutral topics, men attempted to exert their power through dominating the amount of time talking. However, when the topic was feminine, women demonstrated more power-related behaviors by speaking longer. This finding extends upon the study of Martin and Craig (1983) by placing an emphasis on the topic of discussion, not just the gender of the person you are talking to. Although neither of these 
studies directly assessed stereotype threat, being that participants were placed in mixedgender dyads (Martin \& Craig, 1983) and asked to speak on gendered topics (Dovidio et al., 1988), an evaluative context was established that placed participants under conditions of threat. Specifically within the Dovidio et al. (1988) study, topic familiarity (e.g., sewing) put one member of the dyad (e.g., women) at an immediate advantage due to topic familiarity. As such, once participants were put under conditions of threat (women talking about oil changing; men talking about sewing), differences in verbal distinctions in the form of amount of speech were observed.

In addition to investigating distinctions between the amount of speech offered by men and women during interpersonal interactions, researchers have also looked to tentative language as a gender-based language difference. The use of tentative language by communicators is frequently perceived as a sign of low status - lacking power, competence, and intelligence (Carli, 1990; Lakoff, 1975). In Palomares' (2009) study, tentative language is of specific interest as social cues are limited in computer-mediated communication, and language is the primary means of forming impressions of others online (Walther, 1996). In his study, participants were told they were going to initiate an e-mail exchange with a student from another class (the gender of the other student was inferred by their name; e.g., John or Jennifer) on one of five topics: 1) car maintenance, 2) sports, 3) clothing designers, 4) cosmetics, and 5) favorite restaurant. The first two topics were masculine in nature, while the second two were feminine and the last question was gender neutral. Just after composing their email, participants were given a gender salience measure to determine how focused they were on their gender while constructing their message (e.g., "While typing my email, I was thinking about being a 
male/female"). Palomares (2009) hypothesized that the assigned topic would affect how tentative the men and women in the study were. Results indicated that women were more tentative when speaking on masculine topics (i.e., sports) to men, and men were more tentative speaking on feminine topics (i.e., cosmetics) to women. These results were not present in same-sex dyads, only mixed-sex indicating that tentative language use is not fully driven by topic knowledge. Additionally, gender salience partially mediated the influence of topic on tentative language use. This finding highlights that individuals had an increased awareness of their gender identity when communicating with a member of the opposite gender about counter-stereotypical topics (e.g., men talking about cosmetics, women talking about sports). The increase in gender salience observed in Palomares' (2009) investigation is similar to that of stereotype threat - individuals become aware of their group membership when in an evaluative situation where they could confirm a stereotype about their group (McGlone \& Aronson, 2006).

Palomares (2009) noted that men and women exhibit different patterns of tentative language use in dyadic communication when one's gender salience is manipulated, a claim that is consistent with the theory of stereotype threat. To test the effects of stereotype threat in the evaluative context of communication, McGlone and Pfiester (in press) recruited men and women to participate in a domain where individuals have competence concerns. In their experiment, participants were presented with an interpersonal conflict scenario between coworkers and told they would be completing a simulation in which they would offer advice on how to resolve the conflict. Participants were instructed to list as many recommendations as possible during the 90 -second simulation. Prior to beginning the experiment, participants were randomly assigned to 
one of three conditions: 1) participants were told the study was investigating differences in leadership ability, which would be demonstrated by their performance in the simulation (threat condition for women); 2) participants were told the study was examining communication in personal relationships with their performance during the simulation being indicative of their ability to maintain close personal relationships (threat condition for men); and 3) the final group was a control and participants in this condition were only told about the upcoming simulation, no measure of ability was presented in this condition. All participants across the three conditions were told that male and female responses would be compared to assess gender differences. The authors hypothesized that when the simulation was framed as leadership ability (i.e., masculine trait), women would use higher rates of tentative language and lower rates of resolution recommendations relative to the relational maintenance frame (i.e., feminine trait) and control groups. The same was hypothesized for men in the relationship maintenance condition. As hypothesized, women were more tentative in their language when the simulation was framed as a measure of leadership ability. Men in the relational maintenance condition were also more tentative in their language use than other men in the leadership and control conditions. The evaluative frame did not influence the rate of recommendations by men or women. In a mediation analysis, participant levels of state anxiety (measured using the STAI-6) performed as a partial mediator of stereotype threat and tentative language use. As for the results of this investigation, when the cross-gender stereotype was activated, participant levels of state anxiety increased, which in turn negatively influenced their communicative abilities. This research by McGlone and Pfiester (in press) is one of the first stereotype threat studies to evaluate the actual 
communication of participants, instead of communication simply being a means of completing an assessment. Their research protocol provides a model for conducting stereotype threat research in the domain of communication.

\section{Hypotheses}

The overarching goal of this dissertation is to investigate the influence of stereotype threat in the communicative domain, specifically focusing on the abilities of men working in the female-dominated profession of nursing. Based on prior research, male nurses constitute a population targeted by negative gender stereotypes and therefore are likely susceptible to threat derived from these stereotypes that may influence their professional communication. Steele and Aronson (1995) first introduced the term "stereotype threat" to describe the apprehension individuals experience from the prospect of confirming a negative self-relevant stereotype. In this seminal work, Steele and Aronson (1995) demonstrated that being placed under conditions of threat had a negative influence on the intellectual performance of academically gifted African-American students at Stanford University. Since this investigation, numerous studies have employed stereotype threat theory to help explain race and gender differences. However, as has been outlined in the literature review of this dissertation, a significant focus of these investigations has been in the academic assessment domain. To date, there is a single published manuscript that highlights the threat effects on communication performance in evaluative contexts (McGlone \& Pfiester, in press), yet other studies have demonstrated similar effects. In a study of same- and mixed-gender dyadic computermediated communication, Palomares (2009) noted that women were more tentative when 
speaking to men on masculine topics (i.e., sports) and men were more tentative speaking on feminine topics to women (i.e., cosmetics). These results were not produced in samegender dyads, which indicates that tentative language is not fully driven by topic knowledge.

Extending this line of research, McGlone and Pfiester's (in press) study indicated that both men and women were more tentative in their language use when placed in conditions of stereotype threat. The experimental simulation used in this study tasked participants to address a male or female patient, therefore making it possible to observe the influence of threat conditions as well as same- and mixed-gender dyadic composition on the use of tentative language. Thus, the first goal of this dissertation is to apply a variant of McGlone and Pfiester's (in press) experimental paradigm to further investigate the influence of stereotype threat in interpersonal interactions. Extending on this first goal, the current study also sought to observe the communicative performance (i.e., tentative language and grammatical error use) of male and female nursing students during a procedural simulation that placed an emphasis on the nurse-patient relationship. As noted by Campbell et al. (2007), it is necessary for patients to be given accurate information regarding their health. Accuracy extends beyond diagnoses and prognoses to include the grammatical correctness of presented information. Investigating tentative language and grammatical error rates in the context of nursing, with a heightened focus on nursing as a gendered occupation, is the second goal of this dissertation.

The final goal of this dissertation is to develop an online stereotype threat simulation that affords researchers the opportunity to recruit diverse samples without being centrally located. This study is one of the first to utilize an online methodology to 
investigate stereotype threat and communication performance. Being able to recruit and collect data online offers researchers the ability to collect data efficiently, especially in situations when the sample is recruited from an underrepresented population - i.e., nurses (Couper, 2005).

Based on the current review of the literature, the following hypotheses are offered:

H1: Framing a nurse-patient interaction simulation as diagnostic of a stereotypical feminine ability (empathy) induces stereotype threat in men, as indicated by higher rates of tentative language $\left(\mathrm{H}_{1 \mathrm{~A}}\right)$ and grammatical errors $\left(\mathrm{H}_{1 \mathrm{~B}}\right)$ relative to the non-ability (control) or masculine ability framing (patient management).

$\mathrm{H} 2$ : Framing the simulation as diagnostic of a stereotypical masculine ability (patient management) induces stereotype threat in females, as indicated by higher rates of tentative language $\left(\mathrm{H}_{2 \mathrm{~A}}\right)$ and grammatical errors $\left(\mathrm{H}_{2 \mathrm{~B}}\right)$ relative to the non-ability (control) or feminine ability framing (empathy).

In addition to these hypotheses, the current study also investigated the indirect effects on anxiety, gender-identity salience, nursing identification, and perceived genderrelated work barriers produced by stereotype threat in the evaluative context of communication. Prior studies have suggested that state anxiety partially mediates stereotype threat in the testing domain (Osborne, 2001; Spencer et al., 1999) as well as in communication (McGlone \& Pfiester, in press), so it is hypothesized to mediate the relationship between stereotype threat and the evaluative context of communication. 
Furthermore, gender-identity salience can influence language use, as demonstrated by Palomares' (2009) study of same- and mixed-gender dyads in computer-mediated interactions. Palomares found that women were more tentative when speaking about stereotypically masculine topics and men were more tentative speaking on stereotypically feminine topics in mixed- but not same-gender dyads. These findings also highlighted that gender salience partially mediated the influence of discussion topic on tentative language use. In other words, when talking to members of the opposite gender about a counter-stereotypical topic (i.e., men talking about cosmetics), participants' levels of gender salience increased, ultimately influencing their use of tentative language. Next, this study sought to observe the influence of nursing identification on stereotype threat and communication interaction. Research by Aronson et al. (1999) demonstrated that students who "highly identified" with math performed significantly worse on a math assessment under conditions of threat. Results from their investigation indicate that the degree to which an individual identifies with a domain can influence their susceptibility to stereotype threat. Thus, it is necessary to control for "nursing identification" as a potential moderating variable for the effects of stereotype threat on communication outcomes. Finally, as outlined by Rochlen et al. (2009), male nurses that perceived greater gender-related barriers in the workplace also reported greater levels of conflict, and self-assessed their job skills at a decreased level. Due to the influence gender-related work barriers can have on personal assessments of work performance, it is necessary to control for the potential moderating effects perceived gender-related work barriers can have on the effects of stereotype threat on communicative performance.

These topics are outlined in the following hypotheses: 
$\mathrm{H} 3$ : The effects of evaluative framing on tentative language $\left(\mathrm{H}_{3 \mathrm{~A}}\right)$ and grammatical errors $\left(\mathrm{H}_{3 \mathrm{~B}}\right)$ will be mediated by state anxiety.

$\mathrm{H} 4$ : The effects of evaluative framing on tentative language $\left(\mathrm{H}_{4 \mathrm{~A}}\right)$ and grammatical errors $\left(\mathrm{H}_{4 \mathrm{~B}}\right)$ will be mediated by gender identification.

$\mathrm{H} 5$ : The effects of evaluative framing on tentative language $\left(\mathrm{H}_{5 \mathrm{~A}}\right)$ and grammatical errors $\left(\mathrm{H}_{5 \mathrm{~B}}\right)$ will be moderated by nursing identification.

H6: The effects of evaluative framing on tentative language $\left(\mathrm{H}_{6 \mathrm{~A}}\right)$ and grammatical errors $\left(\mathrm{H}_{6 \mathrm{~B}}\right)$ will be moderated by perceived gender-related work barriers. 


\section{Chapter Three: \\ Method}

\section{Participants}

Students currently majoring in nursing programs across the United States were recruited to participate in the current study. Recruitment announcements were distributed electronically at the University of Texas at Austin, the University of Texas Health Science Centers at Houston and San Antonio, Texas State University, Boston College, and the University of Pennsylvania (see Appendix D for a copy of the participant recruitment letter). Announcements were also distributed to the respective student listservs of the American Assembly for Men in Nursing, the National Student Nurses Association, the American Nurses Association, and the Nursing Student Network. The current study was labeled as "Communication in the Health Setting" in all recruitment messages. Upon receipt of the online recruitment announcement, interested students were guided to Qualtrics, a web-based survey tool where they could participate.

Qualtrics was selected for the current study due to the ease of accessibility and use for participants, and it provided a secure data file that is only accessible by the researcher, ensuring participant confidentiality. Overall, online data collection was used as it has previously demonstrated efficiency in the recruitment of underrepresented and large samples (Couper, 2005). Upon completion of the experiment, participants were given the opportunity to enter a raffle for a chance to win one of ten $\$ 50$ gift cards to Amazon or iTunes.

Purposive sampling was used to control for participant gender. Otherwise, no attempts were made to recruit students in terms of age, race, religion, etc. Four hundred 
and sixteen nursing students completed the experiment. Forty-one participants were excluded from data analysis (see Chapter 4 for omission rationale). The final sample consisted of 375 (192 female, 183 male) current bachelors-level students majoring in nursing. Participant ages ranged from 18 to $49(\mathrm{M}=24.97$ years, $\mathrm{SD}=5.76)$. The average time participants had been nursing students was 12.42 months $(\mathrm{SD}=10.05)$. Just over half (59.5\%) of the participants identified themselves as Caucasian, while $11.2 \%$ identified as African American, 6.7\% as Asian, 5.3\% as Multi-Racial, 4.5\% as Hispanic, $3.2 \%$ as American Indian, $2.7 \%$ as Other, while 2 participants self-identified as MiddleEastern and 24 failed to report racial/ethnic identity. Participants were geographically dispersed around the United States, with $31.7 \%$ of the sample being located in the South, $26.1 \%$ in the Northeast, $24.9 \%$ in the Midwest, and $17.3 \%$ in the West. Finally, $28 \%$ of the sample had medical experience prior to majoring in nursing (e.g., military, CNA, EMT), and $20.8 \%$ had an immediate family member that was a nurse (See Table 1 for the sample's demographic profile).

\section{Design and Procedure}

This experiment employed a 2 × 2 × 3 factorial design with participant gender (male or female), patient gender (male or female), and diagnostic frame (patient management, empathy, or control) as between-participants factors. Upon voluntarily accessing the online survey hosted on Qualtrics, participants read a brief description of the experiment prior to reviewing the study's informed consent (see Appendix C for a copy of the informed consent). The study was described as a research project focused on how healthcare providers interact with patients. After providing informed consent, 
participants began the experiment. The study progressed in two phases, described below.

From the time participants logged in, their sessions took approximately 30 minutes.

Phase 1. Participants were randomly and covertly assigned to one of three evaluative frames (patient management, empathy, or control) for the experiment. A paragraph located at the top of the second page of the online survey served as the primary manipulation for the evaluative frame. In the two experimental conditions (patient management and empathy), the paragraph stressed that the purpose of the current study was to investigate gender differences in ability, along with an explanation of why the procedure under investigation was diagnostic of ability. The final sentence of the manipulation paragraph, which was consistent across all three conditions, reiterated that responses to the upcoming simulation would be compared to other men and women that are participating in the study to test for gender differences. The paragraphs associated with each condition are presented below, with wording specific to the framing manipulation presented in bold (but did not appear in this format to participants).

The patient management condition manipulation read as follows:

The purpose of this research is to investigate differences in nurses' abilities to manage patients. Patient management ability entails understanding a patient's circumstances and acting upon that understanding in order to make the patient feel understood, comfortable, and secure regarding the care being provided. Numerous scientific studies have demonstrated that patient management ability can be predicted from observing people as they simulate the communication behaviors they use when interacting with others. In today's study, you will simulate the interaction you would use when entering an examination room to explain the results of a medical test to a patient. Patient managers must be able to articulate necessary information clearly, so your performance on this simulation provides one way to measure your patient management ability. Your simulation response will be analyzed later on by a team of researchers studying nurses' patient management ability here at the university. They will compare your responses to those of 
other men and women participating in this study to examine gender differences in performance.

While referencing a different ability, the empathy condition is similar to that of the patient management condition in form:

The purpose of this research is to investigate differences in nurse's ability to empathize with patients. Empathy ability entails understanding a patient's circumstances and acting upon that understanding in order to make the patient feel understood, comfortable, and secure regarding the care being provided. Numerous scientific studies have demonstrated that empathic ability critically depends on communication behavior. This research has also found that empathic ability can be predicted from observing people as they simulate the communication behaviors they use when interacting with others. In today's study, you will simulate the interaction you would use when entering an examination room to explain the results of a medical test to a patient. Empathic individuals must be able to articulate necessary information clearly, so your performance in this simulation provides one way to measure your empathy level. Your simulation response will be analyzed later on by a team of researchers studying nurses' empathic ability here at the university. They will compare your recording to those of other men and women participating in this study to examine gender differences in performance.

In contrast to the aforementioned conditions, the control condition introduced the simulation to the participants but did not articulate a purported rationale for diagnosing a specific nursing-related ability:

In today's study, you will simulate the interaction you would use when entering an examination room to explain the results of a medical test to a patient. Your simulation response will be analyzed later on by a team of researchers here at the university. They will compare your simulation response to those of other men and women participating in this study to examine gender differences in performance.

After participants read the framing paragraph, they were instructed to carefully read the procedural simulation scenario described at the bottom of the same page.

Simulation scenario. Research on proactive "imagined interaction" theory (Honeycutt, 2003, 2008, 2010) suggests that individuals commonly imagine upcoming interactions to help organize thoughts and adapt their message strategy for that 
forthcoming context. Simulation-based training methods like those used in nursing programs follow Honeycutt's $(2003,2008,2010)$ premise to make sure healthcare practitioners are accurately and safely providing medical care to their patients (Berwick \& Leape, 1999; Feinstein, 1997; Ziv, Wolpe, Small, \& Glick, 2003). As noted by researchers, it is common for nursing students to rehearse patient interactions by participating in individual-based and dyadic role-playing simulation (Barrows, 1993; Simpson, 1985; Ziv, Small, \& Wolpe, 2000). These simulations commonly occur via computer-based simulations (Ralston, Rutter, Carrell, Hecht, Rubanowice, \& Simon, 2009; Weis \& Guyton-Simmons, 1998). Moreover, scholars have noted benefits in the communicative behaviors for those practitioners that have participated in computer-based patient communication simulations (e.g., Wagner, Pfeiffer, \& Harrington, 2011).

To establish the simulation scenario, three practicing Registered Nurses (RNs) that recently graduated from their respective undergraduate institutions (within the previous 5 years) were interviewed. During the interviews, the focus was on communication-based patient simulations they completed as part of their nursing curriculum. Similar to published simulations (see Comer, 2005; Gaberson \& Oermann, 1999), all three RNs discussed the use of role-playing cases where they were asked by a physician to deliver a minor patient diagnosis (e.g., influenza, strep throat) and the necessary medication information. Based on the information obtained during these interviews and from reviewing published research, the current simulation scenario was selected. To make sure the simulation being used in the current study was accurate to their descriptions, a copy of the simulation was provided to each of the initial interviewees for validation. Additionally, the simulation was distributed to twelve 
separate RNs who attended various academic institutions to verify the validity of the simulation in relation to what they completed during their education. All twelve RNs verified to have either completed the exact simulation or one very similar to it. The simulation scenario will be described below, as well as in Appendix E.

The scenario described a male (James Jones) or female (Amy Jones) patient (names were randomly and covertly alternated among participants) who visited the doctor's office complaining of high fever, fatigue, headache, and body aches. Participants were instructed to assume the role of a nurse assisting the attending physician (physician gender was never revealed). As per the simulation instructions, the participant was then instructed by the physician to inform the patient of their diagnosis, their prescribed medication, how to properly administer the medication, and potential side effects. Additional instructions were provided to participants asking them to follow the "AIDET" framework during their simulated interactions (see Appendix E for a sample scenario).

AIDET is an acronym commonly used in healthcare to help medical professionals communicate more effectively and efficiently with patients and their families (Boehm, 2008; Reynolds, 2009; Studer Group, 2007). AIDET stands for acknowledgement, introduction, duration, explanation, and thanks. For a detailed explanation of each component of the AIDET framework, see Appendix F. By following the AIDET framework, participants were provided a basic structure on how to approach the simulation - plus it helped with consistency among participants. The instructions included in this section encouraged participants to be as specific as possible so that the patient will have a greater understanding of their expectations. After reading the scenario, 
instructions were provided that reminded participants that their responses would be used to assess gender differences between participants.

Instructions informed participants to take 5 minutes to review the procedural paragraph and prepare for a 3-minute simulation of how they would interact with their patient. This 5-minute preparation period allowed participants to prepare as if they were actually going to enter a room and interact with the patient. During this process, participants were instructed to not write any notes to be used during the simulation. After the preparation period, participants proceeded to the next page of the survey and completed the State Anxiety Inventory (STAI-6; Marteau \& Bekker, 1992). The STAI-6 was inserted as an interruption task to take the minds of the participants off the upcoming simulation. After completing the 6-item state anxiety questionnaire, participants continued to the next page of the survey, where they completed the simulated interaction by typing out their response. Upon completion of the simulation, participants proceeded to the second phase of the study.

Phase 2. In the second phase of the study, participants completed the Personal Report of Communication Apprehension measure (McCroskey, 1982; McCroskey et al., 1985), the Conformity to Masculine Norms Inventory-46 (Parent \& Moradi, 2009), the Conformity to Feminine Norms Inventory-45 (Parent \& Moradi, 2010), the modified Nursing Identification scale (Mael \& Ashforth, 1992), the Gender-Related Work Barriers Inventory (Rochlen et al., 2009), and a demographics questionnaire. Each of these measures, along with the STAI-6 completed during Phase 1, are described in more detail below. 
Personal report of communication apprehension (PRCA-24). The PRCA-24

was administered to measure participant levels of trait-based communication apprehension (McCroskey, 1982; McCroskey et al., 1985). Communication apprehension manifests itself in both physical and emotional anxiety. The PRCA-24 is a 24-item Likert-type assessment of trait-based communication across four contexts: public, small group, meeting, and interpersonal. The instrument is based on a 5-point scale anchored by: $1=$ Strongly Disagree and $5=$ Strongly Agree. To score the PRCA-24, the sum of all 24 items is calculated. Scores ranging from 80-120 indicate high levels of communication, 51-79 indicates a moderate level of communication apprehension, and 24-50 indicates a low level of communication apprehension (McCroskey et al., 1985). As per McCroskey et al. (1985), the overall PRCA-24 score is more reliable $(\alpha=.91)$ and predictive of communication apprehension than scores form the context subscales individually. Therefore, only the overall score of the PRCA-24 was used in the current study. The PRCA-24 scale was reliable $(\alpha=.96)$ and the mean score in the current sample was $62.41(\mathrm{SD}=19.45)$.

In the current study, communication apprehension served as a covariate to account for dependent measure variance not attributable to the experimental manipulation. Prior research has documented that communicators experience anxiety in highly evaluative contexts (e.g., Booth-Butterfield \& Butterfield, 1986; Daly \& Buss, 1984), which has the potential to influence participant performance in this current study. See Appendix G for a copy of this measure.

State anxiety inventory. The State-Trait Anxiety Inventory (Spielberger, Gorsuch, \& Lushene, 1970) is a commonly used instrument to assess participant levels of 
state and trait anxiety. Trait anxiety is an individual's stable personality, while state anxiety fluctuates from this stability based on external stimuli. Being that it is important to control for the emotional state of participants at the time of the experiment, only state anxiety was measured. The original anxiety inventory consisted of 40 Likert-type items anchored on a 4-point scale by: $1=$ Not At All and 4= Very Much So. Since the inception of the original State-Trait Anxiety Inventory (STAI), a 6-item shortened version has been introduced (STAI-6). As per Marteau and Bekker (1992), no significant differences in mean scores between the full form STAI and the STAI- 6 have been observed. The six items of the STAI-6 ask participants to identify how they currently feel in relation to feeling calm, tense, upset, relaxed, content, and worried. In a study of the anxiety levels of pregnant women after receiving normal or abnormal prenatal test results, Marteau and Bekker (1992) reported a mean score of $46.4(S D=14.8)$ for the full form STAI and a transformed mean score of $47.7(S D=15.8)$ for the STAI-6. This research by Marteau and Bekker (1992) helps to demonstrate the similarity of the two inventories in terms of mean scores and standard deviations. To maximize response rates as well as improve time efficiency, the STAI-6 was used in replacement of the full form. The STAI-6 is on the same 4-point Likert-type scale with the same anchors as the 40-item inventory. The STAI-6 scale was found to be reliable $(\alpha=.82)$ and the mean score in the current sample was $10.63(\mathrm{SD}=3.59)$. State anxiety was included in the current study as a potential mediating variable (Baron \& Kenny, 1986), as state anxiety has previously mediated the effects of stereotype threat in standardized testing domains (McGlone \& Pfiester, in press; Spencer et al, 1999). See Appendix H for a copy of this measure. 


\section{Conformity to masculine norms inventory-46 (CMNI-46). The CMNI-46}

(Parent \& Moradi, 2009) is a condensed version of the 94-item Conformity to Masculine Norms Inventory offered by Mahalik et al. (2003) to measure the conformity of individuals to masculine norms. The CMNI-46 is being used in the current study to assess the degree of participant conformity to socially accepted masculine norms. The inventory consists of 46 items on a 4-point Likert-type scale anchored by: $1=$ Strongly Disagree and 4= Strongly Agree, with higher scores indicating increased levels of conformity to masculine norms. There are nine factors represented in the CMNI-46: Emotional Control ("I tend to keep my feelings to myself'), Winning ("In general, I will do anything to win"), Playboy ("If I could, I would frequently change sexual partners"), Violence ("Sometimes violent action is necessary"), Self-Reliance ("It bothers me when I have to ask for help"), Risk-Taking ("I frequently put myself in risky situations"), Power over Women (In general, I control the women in my life"), Primacy of Work ("My work is the most important part of my life"), and Disdain for Homosexuals/Heterosexual SelfPresentation ("I would be furious if someone thought I was gay"). In Parent and Moradi's (2009) initial investigation using the CMNI-46, factor reliabilities ranged from $\alpha=.77-.91$, with an overall coefficient alpha of .88 for the entire inventory. For the current study, the goal was to determine the overall conformity of participants to masculine norms, therefore only the entire inventory was used for analysis instead of individual factors. The CMNI-46 was reliable $(\alpha=.77)$ and the mean score in the current sample was $2.23(\mathrm{SD}=.28)$. Due to irregular factor loadings, four items were omitted from analysis ("I don't like giving all my attention to work," "It would be awful if people thought I was gay," "I never ask for help," and "I would feel uncomfortable if someone 
thought I was gay"). Being that self-identified gender can vary from biological sex, which can alter participant views on men in nursing, the CMNI-46 was included as a potential mediating variable (Baron \& Kenny, 1986). See Appendix I for a copy of this measure and the Conformity to Feminine Norms Inventory.

Conformity to feminine norms inventory-45 (CFNI-45). In addition to the CMNI-46, all participants completed the CFNI-45 (Parent \& Moradi, 2010). The CFNI45 is a condensed version of the 84-item Conformity to Feminine Norms Inventory proposed by Mahalik et al. (2005) to measure the conformity of individuals to feminine norms. The CFNI-45 was used in the current study to assess the degree of participant conformity to socially accepted feminine norms. The inventory consists of 45 items on a 4-point Likert-type scale anchored by: $1=$ Strongly Disagree and 4= Strongly Agree, with higher scores indicating higher levels of conformity to feminine norms. The CFNI-45 consists of 9 factors: Relational ("I believe that my friendships should be maintained at all costs"), Sweet and Nice ("I would be ashamed if someone thought I was mean"), Appearance ("I spend more than 30 minutes a day doing my hair and make-up"), Romantic Relationships ("When I have a romantic relationship, I enjoy focusing my energies on it"), Sexual Fidelity ("I would only have sex if I was in a committed relationship like marriage"), Involvement with Children ("I like being around children"), Domestic ("I clean my home on a regular basis"), Modesty ("I always downplay my achievements"), and Thinness ("I am always trying to lose weight"). In Parent and Moradi's (2010) initial investigation, factor reliabilities ranged from $\alpha=.68-.89$, with an overall coefficient alpha of .79 for the entire inventory. For the current study, the goal was to determine overall level of participant conformity to feminine norms, therefore 
only the entire inventory was used for analysis instead of individual factors. Prior to data collection, the "Appearance" subscale was omitted since it centered on the use of makeup and both males and females would be completing the inventory. The measure was reliable $(\alpha=.75)$ and the mean score in the current sample was $2.87(\mathrm{SD}=.31)$. Six items were deleted from analyses due to irregular factor loadings ("I tell everyone about my accomplishments," "When I succeed, I tell my friends about it," "I hate telling people about my accomplishments," "I always downplay my achievements," "I am not afraid to tell people about my achievements," "I am terrified of gaining weight"). The entire 5item "Modesty" subscale was included in the six items deleted post-data collection due to irregular factor loadings. Being that self-identified gender can vary from biological sex, which can alter participant views on men in nursing, the CFNI-45 is included as a potential mediating variable (Baron \& Kenny, 1986).

Nursing identification inventory. To measure participant levels of domain identification with nursing, a modified version of Mael and Ashforth's (1992) six-item Organizational Identification Scale was used. The scale focuses on the degree to which individuals identify with their organization, and is anchored by: $1=$ Strongly Disagree and 7 = Strongly Agree. While Mael and Ashforth's (1992) efforts focused on organizational identification, more recent studies have modified the scale to address issues of professional identification. Specifically, Hekman et al. (2009) modified the scale to measure the professional identification of physicians. Studies using variations of the organizational identification measure have reported reliability coefficients ranging from $\alpha$ $=.81-.89$. In the current study, the scale was modified to address the degree to which participants identify with nursing as a profession (e.g., "In general, when someone praises 
nurses, it feels like a personal compliment”). The modified Nursing Identification measure was reliable $(\alpha=.83)$ and the mean score in the current sample was $3.11(\mathrm{SD}=$ 1.14). As professional identification has the potential to influence participant performance during the current experiment, the measure was included in the current study as a moderator variable (Baron \& Kenny, 1986). See Appendix J for a copy of this measure.

Gender-related work barriers inventory. To measure perceived professional barriers related to participant gender, the Gender-Related Work Barriers Inventory (GRWB; Rochlen et al., 2009) was used. The GRWB consists of six items on a 5-point scale anchored by: $1=$ Strongly Disagree and $5=$ Strongly Agree. Sample items include “As a male nurse, I experience some obstacles that may not be present for women" and "My gender (i.e., being male) is not an issue in the workplace." The coefficient alpha for the GRWB in the Rochlen et al. (2009) manuscript was .74. For the current study, the GRWB was reliable $(\alpha=.73)$ and the mean score for the current sample was 2.67 (SD $=.71)$. Being that perceptions of gender-based barriers can influence participant performance during the experiment, this measure was included in the current study as a moderator variable (Baron \& Kenny, 1986). See Appendix K for a copy of this measure.

Demographic measure. The demographics of interest were age, gender, racial/ethnic identity, educational status (i.e., length of time as a nursing major; specialization), geographic region where the participant was located, if the participant had prior medical experience (a prompt followed inquiring about specific prior experience if participants responded in the affirmative to having prior medical 
experience), and if a member of the participant's immediate family was a nurse (and their relationship to that individual). See Appendix L for a copy of this measure.

Upon completion of the aforementioned measures, participants proceeded to the last page of the survey where they were asked about any suspicions they had about the study's purpose. Finally, participants were presented with two binary choice questions regarding gender stereotypes associated with being a patient manager and empathic (e.g.,

"Are you aware of the cultural stereotype that portrays one gender as being more empathetic than the other? - Yes or No). If the participant responded in the affirmative, a follow-up question was presented to determine the source of this stereotype (e.g., "According to this stereotype, which gender is supposed to be better at empathy? - Male or Female). All participants, regardless of condition, received the same self-report questionnaires and post-experiment interview questions. After completion of all procedures, participants were debriefed through the online survey, thanked for their participation, and provided a link to a separate page where they could enter their email address for a chance to win one of ten $\$ 50$ gift cards. The entire procedure lasted approximately 30 minutes.

\section{Simulation Coding}

Three sets of two trained research assistants that were naïve regarding the study hypotheses coded the procedural simulation data. Each set of trained research assistants worked independently of one another on a unique variable of interest. The three variables requiring the use of coders will be described in greater detail below.

Tentative Language. As defined by Palomares (2008), tentative language includes the features of hedges (e.g., maybe, sort of, probably, etc.), disclaimers (e.g., I 
may be wrong, I guess, etc.), and tag questions (e.g., wouldn't you?, don't you think?, etc.). In addition to hedges, disclaimers, and tag questions, researchers have acknowledged that tentative language also includes the uses of intensifiers (e.g., it is extremely important that..., your test results are particularly encouraging) and rounders (e.g., you'll need to take this medicine for approximately 5-7 days, you should feel better in about 5 hours). Tentative language was initially coded using Pennebaker, Booth, and Francis' (2007) Linguistic Inquiry and Word Count (LIWC) program. To calculate participant tentative language use rate per 100 words, the total number of hedges, disclaimers, tag questions, intensifiers, and rounders were summed and transformed. To transform the data, the summed tentative language rates per participant were divided by the total amount of words in the transcript for that participant and multiplied by 100 (see Bradac, Mulac, \& Thompson, 1995; Mulac, Lundell, \& Bradac, 1986).

Upon review of the data produced by LIWC, it became clear that the program did not take into account contextual cues that alter the interpretation of words and phrases. LIWC was also not accurate in deciphering between the unique forms of tentative language. Upon careful consideration of the LIWC output, the decision was made that tentative language would be best served if it were coded by hand. Therefore, two trained research assistants coded for tentative language use (see Appendix M for a copy of the Tentative Language Codebook). The data were transformed in the same manner as described in the above paragraph.

Grammatical Errors. This dependent variable consisted of errors in grammar, spelling, and punctuation during the simulation. Studies have demonstrated that when nervous or anxious, individuals uses of grammatical errors increases (Daly \& Miller, 
1975; Guéguen et al., 2009). Additional studies that have addressed this variable include Abu-Rabia (2004), Gregersen (2003), Horwitz (2001), Laljee and Cook (1975), and Lynch and May (1977), to name a few. Two trained research assistants (both with undergraduate degrees in English and currently employed as English teachers at the secondary education level) read the simulation paragraphs and assessed each for the use of grammatical errors. To calculate participant grammatical error use per 100 words, the total number of grammatical errors were summed and transformed. To transform the data, the summed grammatical errors per participants were divided by the total amount of words in the transcript for that participant and multiplied by 100 (see Bradac, Mulac, \& Thompson, 1995; Mulac, Lundell, \& Bradac, 1986). See Appendix N for a copy of the Grammatical Error Codebook.

Empathy. Empathy in the medical field is commonly understood in terms of one's "ability to understand the patient's situation, perspective and feelings, and to communicate that understanding to the patient" (Coulehan et al., 2001). Two research assistants coded the degree of empathy that appeared in each of the transcripts on a scale of 1 (least empathic) to 3 (most empathic; see Appendix $\mathrm{O}$ for the copy of the Empathy Codebook). The empathy coding scheme was developed from a review of research centered on the uses of empathy during medical interactions (Krupat et al., 2006; Pfeil \& Zaphiris, 2007). A case received a score of "1" if the participant merely provided the facts to the patient with no attempt to show concern for that patient:

Hi. I'm your nurse today. Dr. Smith asked me to come speak with you while he takes care of another patient. Based on the tests he ran and your symptoms, he's pretty sure you have the flu. Go ahead and take this medicine, TAMIFLU, and you shouldn't have any symptoms after a week. 
Is there anything I can answer for you? Have a great afternoon James (Case 81)

Cases receiving a " 2 " demonstrated some signs of empathy by asking questions

and/or attempting to ease the mind of the patient:

Hi AMy Jones, dr. Smith tells me you're not feeling well today. If I may ask, what is bothering you? Those symptoms sound a lot like the flu, which the doctor has recommended a medication for. The medicine you'll be taking is TAMIFLU and it is an antiviral, which means it will fight the virus that is ailing you. You'll only be on this for 5 days. SO it is not that bad. Hopefully this makes you feel somewhat better, since htis is not a major sickness. The doctor is making rounds and will be in shortly (Case 260)

Finally, cases received a "3" if the participant delivered multiple empathic statements to the patient that built upon the expectations of a score of " 2 " by really making sure the patient understood that they felt their pain:

Hi Amy, my name is Simon. I'm a nurse working with Dr. Smith today. How are you doing? / I'm sorry you're not feeling well. I know having the flu is the worst. Dr Smith let me know ther eis a medication we can offer you that can help you feel better faster. It's called Tamiflu - have you ever heard of it? / Right, it's a medication that fights the flu virus. It's works for stomache flu as well as other types of flu. You take it twice a day. One pill twice a day for five days. So that's one pill in the morning and one in the evening. Yes, take it for the full five days. You don't have to take it with food but my reccomendation is that you do. One of the side effects of the medication is nausea, but it's much better if you take the pill with food. I know, nausea is ecactly what you are tyring to avoid. But I think that if you are sure to take the pills with food you will have no nauseau or just ver minor nausea, and you should feel better faster than if you didn't take it. That's a good question. We can run that by Dr. Smith when he comes back in. Is there anything else I can help you with in the meantime? Can I get you a glass of water or a new magazine? Okay, I will go let Dr. Smith know you're ready to see him again. Thanks so much for coming to see us today (Case 261) 
As certain individuals are predispositioned to be more or less empathic during interactions (Egbert \& Parrott, 2003; Hubbard, 2001), empathy was included in the current study as a potential covariate. 


\section{Chapter Four:}

\section{Results}

Forty-one participants were excluded from data analysis who a) did not properly follow the experimental simulation instructions $(\mathrm{n}=21$; e.g., left the simulation blank, noticeably did not take the simulation serious), b) did not provide an academic email address to verify enrollment as a nursing major $(\mathrm{n}=12)$, and $\mathrm{c}$ ) claimed to be unaware of the gender stereotype associated with empathy, females, and nursing in the postexperiment questionnaire $(\mathrm{n}=8)$. These participant eliminations brought the sample to the current 375 participants, all of which followed the experimental instructions properly and appeared to be naïve regarding the study's hypotheses.

\section{Coding}

To evaluate evidence for the proposed hypotheses, three sets of two trained research assistants naïve to the research hypotheses coded the procedural simulation data for tentative language (disclaimers, hedges, tag questions, intensifiers, and rounders), the amount of grammatical errors per participant simulation response, and the degree of empathy per participant (empathy is functioning as a covariate in the current study). The trained research assistants within each pair worked independently of one another with separate copies of identical transcripts. Workshops were conducted with each pair of research assistants in which definitions of terms and examples of variables were reviewed, thereby ensuring that the research assistants could satisfactorily identify the variables of interest. Upon completion of the workshops, the research assistants were instructed to code twenty-five percent of the cases to be able to calculate intercoder agreement. The 
rate of agreement for each pair of research assistants was good (Krippendorff, 2004; Riffe, Stephen, \& Fico, 2005): tentative language (Cohen's $\kappa=.88)$; grammatical errors (Cohen's $\kappa=.90)$; empathy (Cohen's $\kappa=.92)$. The remaining seventy-five percent of the cases were then distributed equally between the research assistant pairs. Once all cases were coded, the frequency of tentative language and grammatical error use were transformed (divided by the total number of words and multiplied by 100) to produce a percentage per participant in relation to the amount of words typed.

\section{Diagnostic Framing Effects}

The performance effects predicted by $\mathrm{H}_{1}$ and $\mathrm{H}_{2}$ were tested in a $2 \times 2 \times 3$ multivariate analysis of covariance with participant gender, patient gender, and diagnostic frame as independent variables (see Appendices $\mathrm{P}$ - AA for representative participant simulation responses); trait communication apprehension (PRCA-24 score) and empathy as covariates; and tentative language and grammatical errors as dependent variables. All cells ranged from 30 to 36 participants (see Table 1 for participant cell distribution).

Results from evaluations of normality of sampling distributions, homogeneity of variance, and multicollinearity were all satisfactory.

The omnibus test did not reveal a significant main effect for participant gender $(F$ $(2,361)=1.042, p=.35)$, patient gender $(F(2,361)=.039, p=.96)$, or diagnostic frame $(F(4,722)=.657, p=.62)$. The MANCOVA main effect was moderated by a significant participant gender by diagnostic frame interaction, $F(4,722)=2.525, p=.04$, $\eta_{\mathrm{p}}{ }^{2}=.014$. Trait communication apprehension was not a significant covariate, $F(4,342)$ $=.744, p=.56$ and so was omitted from future analyses. Empathy was as a significant 
covariate, $F(4,358)=16.978, p<.01, \eta_{\mathrm{p}}{ }^{2}=.159$. One-tailed planned comparisons were conducted to identify the predicted mean differences (Keppel, Saufley, \& Tokunaga, 1989). The mean tentative language and error rates by participant gender, patient gender, and diagnostic frame are presented in Table 2.

\section{Evaluative Framing Effects}

\section{H1: Empathy Framing and Male Participants}

$\mathrm{H}_{1}$ predicted that framing the experimental interaction simulation as diagnostic of a stereotypically feminine ability (empathy) would induce stereotype threat in male participants, as indicated by higher rates of tentative language $\left(\mathrm{H}_{1 \mathrm{~A}}\right)$ and grammatical error use $\left(\mathrm{H}_{1 \mathrm{~B}}\right)$, relative to the masculine ability (patient management) and non-ability (control) frames. Planned contrasts were used to test for differences in $\mathrm{H}_{1}$. For $\mathrm{H}_{1 \mathrm{~A}}$, there was not a significant difference in tentative language use for male participants in the empathy frame $(M=5.05, S D=3.58)$ compared to male participants in the patient management $(M=4.92 . S D=2.67)$ or control frame conditions $(M=4.66, S D=2.76), F$ $(2,176)=.454, p=.64)$. However, taking into account patient gender, male participants in the empathy frame with a female patient $(M=4.13, S D=2.50)$ were significantly less tentative than male participants in the patient management frame with female patients $(M$ $=5.23, S D=2.36)$ but not with female patients in the control frame $(M=4.71, S D=$

2.65), $F(2,88)=3.449, p=.01, \eta_{\mathrm{p}}{ }^{2}=.073$. Additionally, male participants in the empathy frame with a male patient $(M=6.00, S D=4.27)$ were significantly more tentative than male participants in the patient management frame with a male patient $(M$ 
$=4.61, S D=2.94)$ and male participants with a male patient in the control frame $(M=$ 4.60, $S D=2.91), F(2,87)=3.028, p=.05, \eta_{\mathrm{p}}^{2}=.033$.

When analyzing the presence of grammatical errors by male participants during the simulation $\left(\mathrm{H}_{1 \mathrm{~B}}\right)$, there was a significant main effect for diagnostic frame. Male participants in the empathy frame $(M=6.10, S D=4.48)$ used significantly more grammatical errors than male participants in the patient management frame $(M=5.19$, $S D=3.18)$, but less than male participants in the control frame condition $(M=7.64, S D=$ 5.85), $F(2,176)=4.178, p=.02, \eta_{\mathrm{p}}^{2}=.045$.

\section{H2: Patient Management Framing and Female Participants}

$\mathrm{H}_{2}$ predicted that framing the interaction simulation as diagnostic of a stereotypically masculine ability (patient management) would induce stereotype threat in female participants, as indicated by increased rates of tentative language $\left(\mathrm{H}_{2 \mathrm{~A}}\right)$ and grammatical error use $\left(\mathrm{H}_{2 \mathrm{~B}}\right)$ relative to feminine ability (empathy) or non-ability (control) framing. For $\mathrm{H}_{2 \mathrm{~A}}$, a planned contrast did not reveal a significant difference between female participants' rate of tentative language in the patient management frame $(M=5.69$, $S D=3.12)$ as compared to female participants in the empathy frame $(M=5.27, S D=$ 3.33), and control frame conditions $(M=5.33, S D=3.09), F(2,185)=.291, p=.75)$, regardless of patient gender. $\mathrm{H}_{2 \mathrm{~B}}$ also was not supported, as female participants in the patient management frame $(M=6.43, S D=4.69)$ did not make significantly more grammatical errors than female participants in the empathy frame $(M=6.46, S D=4.49)$ and control frame conditions $(M=5.45, S D=3.48), F(2,185)=1.076, p=.34$. 


\section{Multiple Mediation Analysis}

Hypotheses 3 and 4 assume that state anxiety $\left(\mathrm{H}_{3}\right)$ and gender identification $\left(\mathrm{H}_{4}\right)$ are heightened by stereotype threat associated with specific evaluative frames. To investigate these claims, participant gender and evaluative frame were recoded into a single variable to reflect stereotype consistent interactions. The resulting "stereotype frame" consisted of three levels: 1) stereotype-neutral frame (non-ability), 2) stereotypeconsistent frame (men evaluated for patient management ability, women for empathic ability), and 3) stereotype-inconsistent frame (men evaluated for empathic ability, women for patient management ability). To assess these two hypotheses, a mediation model was established to examine the potential influence of state anxiety and gender identification on stereotype threat frame and the dependent variables (Kenny, Kashy, \& Bolger, 1998; Preacher \& Hayes, 2008). Because there is a potential for the mediators to overlap with one another, a multiple mediation was analysis was conducted (Preacher, Rucker, \& Hayes, 2007) instead of independent simple mediation models. Therefore, the hypothesized multiple mediation model explored not only indirect effects between the independent and dependent variables but also the relationship between the mediating variables. The indirect.sbs macro bootstrapping method (Preacher \& Hayes, 2008) was used in this analysis because it allows for the statistical control of covariates while also considering possible pair-wise comparisons between indirect effects. The results of the multiple mediation analysis are presented in Figure 1.

\section{H3: State Anxiety as a Mediator}

$\mathrm{H}_{3}$ predicted that state anxiety would mediate the effects of evaluative framing on tentative language $\left(\mathrm{H}_{3 \mathrm{~A}}\right)$ and the presence of grammatical errors $\left(\mathrm{H}_{3 \mathrm{~B}}\right)$. Regression 
analyses were conducted to establish the key conditions for state anxiety's mediation of stereotype frame effects on the study's dependent variables: stereotype frame was not associated with either tentative language $(\beta=.025, t(373)=.490, p=.62)$ nor grammatical error use $(\beta=.073, t(373)=1.417, p=.16)$; stereotype frame was not associated with state anxiety $(\beta=.046, t(373)=.885, p=.38)$; and state anxiety was not associated with either tentative language $(\beta=-.062, t(373)=-1.192, p=.23)$ or grammatical error use $(\beta=-.088, t(373)=-1.702, p=.09)$. Controlling for state anxiety in the multiple mediation model, all paths were still non-significant: stereotype frame was not associated with tentative language $(\beta=.068, t(373)=.362, p=.71)$ or grammatical error use $(\beta=.398, t(373)=1.410, p=.15)$; stereotype frame was not associated with state anxiety $(\beta=.203, t(373)=.899, p=.37)$; and state anxiety was not associated with tentative language $(\beta=-.056, t(373)=-1.276, p=.20)$ or grammatical error use $(\beta=$ $.117, t(373)=-1.812, p=.07)$. Thus the evidence from this analysis does not support the study hypotheses, as state anxiety does not appear to mediate the relationship between stereotype frame and tentative language $\left(\mathrm{H}_{3 \mathrm{~A}}\right)$ or grammatical error $\left(\mathrm{H}_{3 \mathrm{~B}}\right)$ use in the current study.

\section{H4: Gender Identification as a Mediator}

$\mathrm{H}_{4}$ predicted that gender identification would mediate the effects of evaluative framing on tentative language $\left(\mathrm{H}_{4 \mathrm{~A}}\right)$ and the presence of grammatical errors $\left(\mathrm{H}_{4 \mathrm{~B}}\right)$. Again, regression analyses were conducted to establish the key conditions for gender identity's mediation of stereotype frame effects on the study's dependent variables: stereotype frame was not associated with either tentative language $(\beta=.025, t(373)=.490, p=.62)$ 
or grammatical error use $(\beta=.073, t(373)=1.417, p=.16)$; stereotype frame was not associated with gender identity $(\beta=-.028, t(373)=-.546, p=.56)$; and gender identity was not associated with either tentative language $(\beta=-.001, t(373)=-.028, p=.98)$ or grammatical error use $(\beta=-.031, t(373)=-.591, p=.56)$. Controlling for gender identification in the multiple mediation model, all paths were still non-significant: stereotype frame was not associated with tentative language $(\beta=.068, t(373)=.362, p$ $=.71)$ or grammatical error use $(\beta=.398, t(373)=1.410, p=.15)$; stereotype frame was not associated with gender identity $(\beta=-.010, t(373)=-.551, p=58)$; and gender identity was not associated with tentative language $(\beta=.008, t(373)=.015, p=.99)$ or grammatical error use $(\beta=-.404, t(373)=-.509, p=.61)$. The results from this analysis do not support the study hypotheses. Gender identity does not appear to mediate the relationship between stereotype frame and tentative language $\left(\mathrm{H}_{4 \mathrm{~A}}\right)$ or grammatical error $\left(\mathrm{H}_{4 \mathrm{~B}}\right)$ use.

\section{Moderation Analyses}

\section{$\mathrm{H}_{5}$ : Nursing Identification as a Moderator}

To examine the relationship between stereotype frame (independent variable) and nursing identification (moderator) in predicting participant uses of tentative language $\left(\mathrm{H}_{5 \mathrm{~A}}\right)$ and grammatical errors $\left(\mathrm{H}_{5 \mathrm{~B}}\right)$, a moderated regressions analysis was conducted. For Step 1, stereotype frame and nursing identification were entered. On Step 2, a twoway interaction term (stereotype frame X nursing identification) was entered. 
$\mathbf{H}_{5 \mathrm{~A}}$ : Tentative Language. On Step 1, stereotype frame and nursing identification accounted for less than $1 \%$ of the variance on tentative language use, $F(2,372)=.433, p$ $=.65$. Additionally, neither stereotype frame $(\beta=.024, t(372)=.468, p=.64)$ nor nursing identification $(\beta=-.041, t(372)=-.791, p=.43)$ was significant on the tentative language use in Step 1. On Step 2, the addition of the two-way interaction (stereotype frame $\mathrm{X}$ nursing identification) again explained less than $1 \%$ of the variance, $F(1,371)=$ $1.369, p=.24$. Thus, the two-way interaction of stereotype frame and nursing identification was not significant $(\beta=-.061, t(371)=-1.170, p=.24)$ in explaining participant uses of tentative language.

$\mathbf{H}_{5 \mathrm{~B}}$ : Grammatical Errors. On Step 1, stereotype frame and nursing identification accounted for less than $1 \%$ or the variance on grammatical error use, $F$ ( 2 , $372)=1.036, p=.36$. Additionally, neither stereotype frame $(\beta=.074, t(372)=1.422, p$ $=.16)$ nor nursing identification $(\beta=.013, t(372)=.260, p=.79)$ was significant on the grammatical error use in Step 1. Further, the two-way interaction of stereotype frame X nursing identification again accounted for less than $1 \%$ of the variance, $F(1,371)=.693$, $p=.56$, and was not significant in explaining participant uses of grammatical errors during the simulation $(\beta=.006, t(371)=.113, p=.91$. The results from this analysis indicate that nursing identification does not function as a moderating variable in the current model.

\section{$\mathrm{H}_{6}$ : Perceived Gender-Related Work Barriers as a Moderator}

To examine the relationship between stereotype frame (independent variable) and perceived gender-related work barriers (moderator) in predicting participant uses of tentative language $\left(\mathrm{H}_{6 \mathrm{~A}}\right)$ and grammatical errors $\left(\mathrm{H}_{\mathrm{B}}\right)$, a moderated regression analysis 
was conducted. For Step 1, stereotype frame and perceived gender-related work barriers were entered. On Step 2, a two-way interaction term (stereotype frame X perceived gender-related work barriers) was entered.

$\mathbf{H}_{6 \mathrm{~A}}$ : Tentative Language. On Step 1, stereotype frame and perceived genderrelated work barriers accounted for less than $1 \%$ of the variance on tentative language use, $F(2,372)=.900, p=.41$. Additionally, neither stereotype frame $(\beta=.024, t(372)$ $=.458, p=.65)$ nor perceived gender-related work barriers $(\beta=.065, t(372)=1.249, p$ $=.21$ ) was significant on the tentative language use in Step 1. On Step 2, the addition of the two-way interaction (stereotype frame $\mathrm{X}$ perceived gender-related work barriers) again explained less than $1 \%$ of the variance, $F(1,371)=1.483, p=.22$. Thus, the twoway interaction of stereotype frame and nursing identification was not significant $(\beta=$ $.063, t(371)=-1.218, p=.22)$ in explaining participant uses of tentative language.

$\mathbf{H}_{6 \mathrm{~B}}$ : Grammatical Errors. On Step 1, stereotype frame and perceived genderrelated work barriers accounted for less than $1 \%$ or the variance on grammatical error use, $F(2,372)=1.141, p=.32$. Additionally, neither stereotype frame $(\beta=.072, t(372)=$ $1.402, p=.16)$ nor perceived gender-related work barriers $(\beta=.027, t(372)=.527, p$ $=.59$ ) was significant on the grammatical error use in Step 1. Further, the two-way interaction of stereotype frame $\mathrm{X}$ perceived gender-related work barriers accounted for just over $1 \%$ of the variance, $F(1,371)=.1 .675, p=.19$, and was not significant in explaining participant uses of grammatical errors during the simulation $(\beta=.067, t(371)$ $=1.294, p=.19$. The results from this analysis indicate that the perception of genderrelated work barriers associated with the field of nursing does not function as a moderating variable in the current model. 


\section{Post Hoc Analyses}

In addition to the results for the study hypotheses outlined above, several post hoc analyses were performed to further explore the relationship between participant gender, tentative language, and grammatical error use during the study's experimental simulation. Specifically, the post hoc analyses sought to eliminate the stereotype threat framing categories (patient management, empathy, and control conditions) to determine the influence of just patient gender during the simulation - the potential factor being related to the gender salience of participants based on patient gender. The post hoc analyses followed two investigative paths: 1) the relationship between male participants and patient gender on tentative language and grammatical error use, and 2) the relationship between female participants and patient gender on tentative language and grammatical error use. A series of independent samples t-tests were conducted to determine if there was a statistically significant difference between two groups (participants who conducted the simulation with a male patient and those who conducted the simulation with a female patient) on the dependent measures of interest.

\section{Post Hoc 1: Male Participants and Patient Gender}

Overall, there was not a significant difference in tentative language rates between participants who conducted the simulation with a male patient $(M=4.68, S D=2.52)$ and those who conducted it with a female patient, $t(181)=-.854, p=.39$ (Female Patients: $M$ $=5.07, S D=3.45)$. There was also no difference in the grammatical error rate between 
these groups (Male Patients: $M=6.47, S D=5.17$; Female Patients: $M=6.15, S D=4.23$ ), $t(181)=.453, p=.65)$. See Table 3 for a summary of these mean scores. Although the results trend towards male participants using higher rates of tentative language in the presence of female patients and an increased presence of grammatical errors when addressing male patients, neither of these findings achieved statistical significance. Therefore, in the current study, patient gender does not appear to influence the rate of tentative language and grammatical errors for male participants during the experimental simulation. Additionally, all mediating and moderating analyses yielded non-significant results, indicating that the study's proposed mediators (state anxiety and gender identification) and moderators (nursing identification and perceived-gender related work barriers) did not have an influence on the tentative language and grammatical error use of male participants during the simulation.

\section{Post Hoc 2: Female Participants and Patient Gender}

There was not a significant difference in tentative language rates between participants who conducted the simulation with a male patient $(M=5.77, S D=3.37)$ and those who conducted it with a female patient $(M=5.07, S D=3.45), t(190)=1.450, p$ $=.15$. There was also no difference in the grammatical error rate between these groups (Male Patients: $M=6.07, S D=4.15$; Female Patients: $M=6.14, S D=4.36), t(190)=$ $.113, p=.91$. See Table 4 for a summary of these mean scores. Although the results trend towards female participants using higher rates of tentative language with male patients, this finding did not achieve statistical significance. Additionally, grammatical error use was comparable between the patient gender groups. Therefore, in the current study, patient gender does not appear to have any influential effects on the use of 
tentative language and grammatical errors for female patients during the experimental simulation. Additionally, all mediating and moderating analyses returned non-significant results indicating that the study's proposed mediators (state anxiety and gender identification) and moderators (nursing identification and perceived-gender related work barriers) did not have an influence on the tentative language and grammatical error use of female participants during the simulation.

The findings from the study's hypotheses and post hoc analyses will be further discussed in the following section. 


\section{Chapter Five:}

\section{Discussion}

The overarching goal of this dissertation was to explore the impact of stereotype threat on male nurses' communication behavior. First investigated by Steele and Aronson (1995), stereotype threat research has demonstrated that reminding participants of self-relevant negative stereotypes can significantly decrease one's performance on an evaluative task (e.g., SAT verbal assessment). To date, the vast majority of stereotype threat studies have focused on standardized testing (verbal and math performance, spatial reasoning skills, etc.); few studies have examined the role of stereotype threat in interpersonal communication (e.g., Breckinridge et al., 2011; McGlone \& Pfiester, in press). This goal was accomplished in the present study using an experimental methodology that tasked participant nurses with a patient-interaction simulation. In this respect, the reported project contributed to the stereotype threat research literature by expanding investigation in the communication domain and providing an initial exploration of the phenomenon among nurses.

The second goal of this dissertation was to examine stereotype threat associated with gender-based occupational stereotypes. Of specific interest to this study is the male nurse, as nursing has one of the most lopsided inequality ratios of any profession, and has been traditionally identified as a career for empathic women. Given the prevailing sociocultural stereotypes that portray men as less empathic than women (Aries, 1996;

Broverman et al., 1972; Eagly \& Steffen, 1984; Flannery, 2000; Spence et al., 1974) and nursing as a career focused on empathy (Davies, 1995), do male nurses experience stereotype threat due to their minority standing in the occupation? Research has 
documented the stereotypes and gender-based barriers faced by men who choose to enter nursing (e.g., O’Lynn, 2004; Spcihiger et al., 2005), but has not demonstrated potential negative communicative outcomes men experience as a consequence of these stressors.

Due to their minority status in the field, there is a potential for male nurses to be susceptible to stereotype threat under conditions that focus on nursing as a caring profession. The reported project put this proposition to the test. Participants were randomly and covertly assigned to one of three experimental conditions that framed nursing performance as attributable to different dispositional abilities (patient management, empathy, or control) to determine the frames' impact on their communicative performance. Based on prior research focused on male and female attributes, the two "threat" conditions (female threat: patient management and male threat: empathy) were identified and subtly manipulated in the current study. The results of the study point to important issues to be considered in future investigations of barriers and stereotypes faced by male nurses.

The final goal of this dissertation was to develop an online stereotype threat simulation that affords researchers the opportunity to recruit samples that are not centrally located - with this study being one of the first to assess communicative performance and stereotype threat using an online methodology. By using such a methodology, participant recruitment was more efficient and required fewer resources than an in-person experiment. Specifically, this study recruited 183 male and 192 female nursing students in less than a month. The size and speed of sampling would not have been possible if participant recruitment was limited to in-person in Austin, Texas. Being able to recruit and collect data electronically across the United States increased the 
efficiency of data collection while also offering the opportunity to increase the study sample size. In addition to addressing these three larger goals, six hypotheses were tested that were derived from the relevant literature. Each hypothesis and the relevant results are summarized below.

\section{Summary of Findings}

The first hypothesis compared tentative language and grammatical error rates between male participants in the threat (empathy), non-threat (patient management), and control conditions. It was predicted that men who received the stereotypical feminine ability prime (empathy) would exhibit higher rates of tentative language $\left(\mathrm{H}_{1 \mathrm{~A}}\right)$ and grammatical errors $\left(\mathrm{H}_{1 \mathrm{~B}}\right)$ relative to those in the masculine ability (patient management) and non-ability control primes. A three-way interaction of the manipulated variables (participant gender $\mathrm{x}$ patient gender $\mathrm{x}$ frame) was not significant and thus the findings were not consistent with the hypothesis. Further investigation highlighted a two-way interaction between condition and patient gender: male participants in the empathy frame with a female patient were significantly less tentative than male participants in the patient management frame with a female patient. Although statistically significant, this finding contradicts the pattern of results predicted. Thus, this finding suggests that the disruptive potential of making salient stereotyped attributes (e.g., empathy) may be offset by patient gender. As noted by Hajek et al. (2004), message recipients are the most influential factor in interpersonal interactions, a statement that has garnered support in this study with patient gender influencing the impact of stereotype threat frame. Therefore, it appears as though the patient gender factor might have complicated the impact of the 
“empathy" prime on participants' performance. Focusing just on participants who conducted the simulation visualizing male patients, male nurse participants in the empathy frame were significantly more tentative than those in the patient management and control frames with a male participant. This finding provides partial support for the first hypothesis as the intergroup component of interaction has been removed from the analysis. Thus, male participants were less tentative with female patients when in the empathy condition, and with male patients in the patient management condition.

Individual analyses of each type of tentative language (disclaimers, hedges, tag questions, intensifiers, and rounders) did not yield any differential impacts of stereotype threat on particular varieties of tentative language, and so tentative language was subsequently considered only in the aggregate.

The second part of the first hypothesis pertained to the presence of grammatical errors during the simulation by male participants. It was predicted that male participants in the empathy frame (under threat) would demonstrate higher rates of grammatical errors relative to those in the patient management (non-threat) and non-ability control conditions. The predicted three-way interaction did not occur; however, there was a significant main effect for diagnostic frame (patient management, empathy, control). Male participants' simulation responses contained fewer grammatical errors in the patient management frame than in the empathy frame. Additionally, male participants' rate of grammatical errors was highest in the control condition, a finding not consistent with the hypothesis. These results provide partial support for the hypothesis, as male participants were more grammatically incorrect in the empathy frame (threat) than the patient management frame (non-threat). This finding demonstrates that male nursing students 
appeared more grammatically fluent in their typed responses in the non-threat condition (patient management) than in the threat condition (empathy). Given that all forms of communication are important for the nurse-patient relationship to be successful, written communication cannot be overlooked as less important than the spoken word (Kerr, 2002). Moreover, the shift in healthcare towards "e-health", which requires nurses to modally communicate with patients via text-based computer-mediated communication (Wickramasinghe, Fadlalla, Geisler, \& Schaffer, 2005), offers an important emerging context for considering the current results.

The second hypothesis compared tentative language and grammatical error rates between female participants in the threat (patient management), non-threat (empathy), and control conditions. It was predicted that women who received the stereotypical masculine ability prime (patient management) would demonstrate higher rates of tentative language $\left(\mathrm{H}_{2 \mathrm{~A}}\right)$ and grammatical errors $\left(\mathrm{H}_{2 \mathrm{~B}}\right)$ relative to those in the feminine ability (empathy) and non-ability control primes. The three-way interaction did not attain statistical significance. The standard deviations for the means compared in this analysis were far higher than expected, suggesting that there was not adequate statistical power to detect the hypothesized differences. Therefore, the possibility exists that the interaction occurred but that there was not sufficient methodological sensitivity to detect its full extent. With that qualification, it nevertheless should be noted that the two-way interaction of participant gender $\mathrm{x}$ frame was in the hypothesized direction. Women were most tentative in the patient management frame (threat condition) and least tentative in the empathy frame (non-threat condition). Finally, female participants were more tentative in their language use than were male participants - this finding is just outside the 
parameter for being considered statistically significant $(p=.08)$. This finding is consistent with those reported by Palomares $(2004,2008)$ indicating that tentative language is schematically more frequent among women than men.

Part two of the second hypothesis focused on the presence of grammatical errors during the simulation by female participants. It was predicted that female participants in the threat condition (patient management) would demonstrate higher rates of grammatical errors relative to those in the feminine ability (empathy) and non-ability control primes. This hypothesis was not supported, as female participants used a comparable amount of grammatical errors in both the patient management and empathy conditions.

Overall, there was only partial support for the first two hypotheses. Male participants with male patients were more tentative in the empathy frame than those in the patient management and control frames. Additionally, the simulation responses contained more grammatical errors for male participants in the empathy frame than in the patient management frame. Female participants, on the other hand, were more tentative in the patient management frame than in the empathy frame. Although these findings are partially consistent with the study's hypotheses, other findings contradicted them. When completing the simulation with female patients, male participants were less tentative in the empathy frame than the tentative language frame, the opposite of what was predicted. Given that the results of this analysis did not correspond with my theoretically derived hypotheses, I offer four post-hoc but nonetheless plausible explanations for why this pattern of findings was observed.

Frame Subtlety. The first possible explanation is that participants did not identify with the constructs of patient management and empathy - especially with the 
stereotype that men are seemingly lacking in empathic potential relative to women. If participants do not identify with the domain within which the threat is being elicited, in this case patient management and empathy, there is a potential that those participants will not experience the disruptive effects of stereotype threat. For example, men are socialized in such a way that they are taught to avoid forms of emotional expression (Chaplin et al., 2005), especially emotions that are not based around masculinity. In fact, there is a possibility that over time, men are actually unable to recognize and identify emotional expressions (Chaplin et al., 2005). Due to this potential factor, it is possible that male participants were not able to fully comprehend "empathy" as a term. This possibility complicates the capacity of the study to detect a difference between men in the threat and non-threat conditions.

A similar explanation could be offered to identify why female participants did not behave as predicted. Research by Ali et al. (1998) and Larew et al. (2006) demonstrates the development of patient management skills during the use of clinical simulations. Moreover, Steadman et al. (2006) stressed the acquisition of patient management ability as a necessary skill set for upper-division nursing majors that will greatly benefit them when they enter the field. Within these recently cited studies, the focus of patient management is not only centered on the physical health of the patient but also their emotional needs and adjustment. Due to this relationship between "patient management" and "empathy", there is the potential that the frames were not sufficiently differentiated. Especially if nursing students are encountering the "patient management" frame in the classroom and clinical settings, some of the terms gendered identification is potentially compromised. The framing used in this experiment may simply have been too subtle to 
induce stereotype threat in the sample. Thus, it might be better to use framing language that transparently invokes the relevant stereotypes (e.g., women are more empathic than men, men are more managerial than women).

Domain-Identification. A second potential explanation for the reported findings is that participants did not strongly identify with the domain of nursing. Domainidentification requires that an individual perceives the selected domain (e.g., nursing) as attractive, important, and having favorable outcomes (Steele, 1997). Moreover, for an individual to identify with a domain, it must be seen as highly self-relevant (Osborne, 1995). As indicated by Crocker, Major, and Steele (1998), stereotype threat is "felt most by people who care or who are invested in the domain where the stereotype threat applies" (p. 591). That is, those who strongly identify with a domain are most susceptible to the pernicious effects of stereotype threat (Aronson et al., 1999; Steele, 1997). Therefore, participants highly identified with nursing (those who value the nursing domain) would be expected to have demonstrated more susceptibility to stereotype threat than those not as highly identified. This pattern was not observed. The sample of nursing students in this study did not reach the criterion for identifying with the nursing domain, according to pre-test measures. Participants completed the Nursing Identification Inventory (modified version of Mael \& Ashforth, 1992) to measure the degree to which they identified with nursing. The mean response for the inventory was 3.11 on a 7 -point scale. This mean does not clear the 4-point threshold identified by Mael and Ashforth as indicative of identification with the domain of nursing. Based on previous research linking domainidentification and stereotype threat, it would be highly unlikely to observe the effects of stereotype threat if the participants did not strongly identify with the domain. 
Issues with Imagined Interactions. Imagined interaction research suggests that individuals commonly visualize upcoming interactions for organizational and message strategy purposes (Honeycutt, 2003, 2008, 2010) in a variety of professional contexts, including nursing (Barrows, 1993; Berwick \& Leape, 1999; Feinstein, 1997; Ziv, Small, \& Wolpe, 2000; Ziv et al., 2003). Despite the practice of engaging imagined interactions for training nurses, it is possible that participants experienced difficulties in establishing 1) the context of the imagined interaction and 2) the "patient." Research by Berger and Jordan (1992) speaks to the difficulties individuals experience when generating an understanding for imagined interactions. One of the major difficulties surrounding the use of imagined interaction is the comprehension of the interactional context - what physical space is this interaction occurring in, how will the interaction develop, how involved will the patient be, etc. - while developing personal expectations. Given that participants come from diverse academic and professional backgrounds with unique experiences, there is a strong likelihood that all participants were imagining a unique context for which their interaction was taking place. For example, a participant that has previous experience as a Certified Nursing Assistant (CNA) may have visualized an interaction in a doctor's office, while a participant with experience as an Emergency Medical Technician (EMT) may imagine an interaction in a more acute care setting. The setting in which a medical conversation occurs can influence the expectations for how that conversation will occur (Beck, Daughtridge, \& Sloane, 2002). Nurses that work in a physician's office will most likely have a different relationship with their patient than a nurse employed on an Intensive Care Unit floor in a hospital. Thus, it is possible that differing perceptions of the medical setting influenced study results. 
In addition to having to visualize the context of the conversation, participants were put in the difficult position of having to imagine the gender of the patient for whom they would be helping. Porter (2010) noted that one of the most difficult components of imagined interaction is the perception of the other person, in this case, the patient. Participants had to imagine who the patient was, what his/her contribution to the interaction would be, and how s/he would react to certain statements. In a couple of cases, participants took the liberty of actually generating the entire interaction with participant responses, while other participants just typed their part of the interaction. That participants completed this simulation in such different ways indicates that there was likely high variability in the nurse-patient interaction they imagined. Leaving so much up to the imagination of the participant, when it comes to the context and patient, might have rendered the online methodology insufficiently sensitive to detect any potential stereotype threat effects.

Finally, given that imagined interaction-based training is used in healthcare simulations, it is also possible that participants were familiar with the simulation, which could render the prime useless. In other words, they were too comfortable with the simulation for it to induce stereotype threat in the first place. As noted by Steele (1997) and Steele and Aronson (1995), stereotype threat is most likely to manifest itself in tasks that are at least moderately difficult. Upon initial review, it appeared as though a simple simulation task would be enough to demonstrate the influence of stereotype threat. However, upon further consideration, this may not be the case. A panel of nurses screened the simulation before the experiment, and they all responded in the affirmative that they received ample training on this type of simulation through their educational 
experience. If participants had previously completed this type of task in the classroom or clinical setting, and they were confident in their ability to successfully complete it, the task might not have provided enough challenge for the participants to induce stereotype threat.

Gender Identification. The final explanation for why results did not correspond with the study's hypotheses is the gender identification of both male and female participants in the current study. Participants completed both the Conformity to Masculine Norms Inventory (Parent \& Moradi, 2009) and the Conformity to Feminine Norms Inventory (Parent \& Moradi, 2010), and were evaluated in terms of which norms they identified more strongly with. A post hoc analysis on the gender identification of participants indicated that male participants identified more strongly with feminine norms than masculine norms, and identified comparably with female participants on the feminine norms inventory. This finding is consistent with Loughery (2008) in that adherence to the female gender role is a central tenet for male nurses. This observation suggests that the recruited sample might not have included sufficient representation of the male nurse population that is, in theory, most susceptible to stereotype threat - i.e., male nurses who identify strongly with male norms. This is not to say that because male participants identified more strongly with feminine norms that they somehow forgot their masculine identity. It does highlight, however, that male participants did not adequately distinguish themselves form the female participants in terms of gender identification creating a lack of gender identity diversity in the study sample. Rooted in social identity theory (Tajfel, 1981; Tajfel \& Turner, 1986) and self-categorization theory (Turner et al., 1987), individuals possess multiple identities based on their membership in certain social 
groups. At different points in time, these individuals can place more importance on a single identity than at other times. Although it is most likely that all male participants recognized their gender identity as "male," it is plausible that they did not place as much importance on gender status as other occupational groups might. If participants did not consider their gender membership as salient while completing the experiment, they most likely did not experience the threat necessary to observe stereotype threat effects.

Hypothesis 3 predicted that state anxiety would mediate the effects of the evaluative framing (stereotype-consistent, stereotype-inconsistent, and control) on tentative language and grammatical error rates. This hypothesis was not supported. The study's mediation hypotheses presumed that there would be an effect of evaluative framing on language behavior, a relationship that was not observed. Given that these predicted effects did not occur, it is not possible to examine anxiety as a mediator. The results from the first two hypotheses influence not only Hypothesis 3, but the remaining three hypotheses as well. Nevertheless, there has been mixed support for anxiety's role as a mediator of stereotype threat effects (Gonzales, Blanton, \& Williams, 2002;

Schmader, 2002; Spencer et al., 1999; Steele \& Aronson, 1995). Thus, it is possible that participants did not experience any anxiety during the experiment since they were able to complete the experiment on their time, when they wanted, and at a location of their choosing (i.e., home). It is also possible that the STAI-6 was not sensitive to subtle shifts in state anxiety that might have mediated participants' language behavior. Although participants reviewed the framing paragraph prior to completing the anxiety measure, there is a possibility that participants were not experiencing anxiety at that point in the experiment, as the simulation had not started yet. If the measure had been inserted after 
the simulation (or perhaps during), there is a possibility that anxiety scores could have differed. Finally, regardless of scale placement, researchers have noted inconsistencies with self-report anxiety measures in stereotype threat research. As stated by Bosson et al. (2003) in relation to the use of self-report anxiety measures, "had we relied solely on selfreports...we would have obtained little evidence that stereotype threat produces anxiety" (p. 253). Their rationale for this conclusion is that participants may say something different from how they are actually feeling - a component of social desirability. Thus, it is worth entertaining the idea of collecting both objective and subjective (via audio- and or video-recordings) anxiety data in the future to further understand the mediating capacities of state anxiety.

Hypothesis 4 predicted that gender identification would mediate the effects of evaluative framing on tentative language and grammatical error rates. This hypothesis also was not supported. As with Hypothesis 3, the predicted effects did not occur thus not allowing the examination of gender identification as a potential mediating variable. Scholars have experienced uncertainty on how to operationalize the construct of gender identification. Specifically, gender identification-based constructs have bounced around the field of stereotype threat research as potential moderators (Schmader, 2002), covariates (Smith \& White, 2001), and mediators (Leyens et al., 2000). However, it has not garnered significant empirical support at any of these levels. One thing that scholars must remember is that constructions of gender are not constant over time, as gender identification is continually evolving based on personal and social experiences (Halim, Ruble, \& Amodio, 2011; Shapiro, 2007). Therefore, it is possible that the results in the current investigation occurred due to male participants identifying more strongly with 
feminine attributes than masculine attributes at that point in time, thus highlighting a more homogenous sample in terms of gender identification than expected.

Second, it is possible that the method used to identify gender identification contributed to these results. Participants completed both the Conformity to Masculine Norms Inventory (Parent \& Moradi, 2009) and the Conformity to Feminine Norms Inventory (Parent \& Moradi, 2010). After calculating mean scores for participants on both scales, each participant was labeled as masculine or feminine dependent upon which scale they score more highly on. For example, if a participant had a mean of 2.4 on the masculine inventory and a 3.6 on feminine inventory, they were designated as more strongly identifying with feminine norms. The mean difference between inventories could have been more than a full point, as in the example above, or tenths of a point. Forcing participants into one of two categories is potentially problematic. Citing the Bem Sex Role Inventory (1981), participants are classified as masculine, feminine, or androgynous. Androgyny reflects the equal endorsement of both masculine and feminine norms. Bem inserted specific items into her inventory to make sure an androgyny score was available. In fact, in a study of masculinity in female-dominated professions, Galbraith (1992) observed that a significant proportion of male nurses identified as androgynous while less than a quarter of participants identified themselves as masculine. This highlights an important oversight in this study, as the degree of difference between the scales was not taken into account. As a case in point, should a participant be labeled as feminine if they scored a 3.2 on the feminine norms inventory and a 3.1 on the masculine norms inventory? It could be argued that the method undertaken to determine gender identification contributed to the lack of support for this hypothesis. 
Hypothesis 5 predicted that nursing identification would moderate the effects of evaluative framing on tentative language and grammatical error rates. This hypothesis was also not supported. In other words, the degree to which nurses identify with their career was not observed to influence participants' susceptibility to stereotype threat effects. This finding is not a surprise, as the predicted effects of evaluative framing on language use were not observed. Therefore, it is not possible for nursing-identification to function as a moderator for a general effect that was not observed. Additionally, mean scores on the nursing-identification measure indicate that participants in the current study did not have a strong identification with nursing. Researchers have pointed out that nurses do not have to strongly identify with their career to take it seriously and perform at a high level, yet identification is important in the retention of a qualified nursing staff (Apker, Zabava Ford, \& Fox, 2003; Ingersoll, Kirsch, Merk, \& Lightfoot, 2000). In light of this research, it does not appear that nursing identification was influential on the communicative performance of nurses in the stereotype-consistent and stereotypeinconsistent conditions of the current study.

The final hypothesis, Hypothesis 6, predicted that the perception of gender-related work barriers would moderate the effects of evaluative framing on tentative language and grammatical error rates. This hypothesis was not supported. Again, without the initially predicted effect of evaluative frame on communicative performance, moderation is rather difficult to explore. Additionally, the mean barrier perceptions scores for male and female participants did not differ significantly, and did not meet the criteria for genderbased work barrier acknowledgment. Low mean scores on the Gender-Related Work Barriers scale do not indicate agreement with perceptions of work barriers (Rochlen et al., 
2009), which was the case in this study. Thus, it appears that neither male nor female participants perceived work barriers in the nursing profession related to their gender. One explanation for this finding is that participants were asked to self-report perceived barriers. The same explanation that was offered for state anxiety applies here as well participants may perceive gender-related barriers but they did not disclose that. Research by Keogh and O’Lynn (2007) has noted that male nurses are hesitant to disclose the experience of gender barriers in the classroom and workplace. Instead, they remove themselves from the situation as is evidenced by current attrition rates. With these explanations in mind and in its current form, it does not seem as though men or women feel as though they experience barriers related to their gender.

\section{Limitations and Future Directions}

The current study had several methodological limitations. First, the materials designed to induce gender-based stereotype diagnostic frames (patient management, empathy, control) did not appear to induce stereotype threat in the participants. Given this failure, it is important to gain a greater comprehension of whether inappropriate conditions were selected or the primes contained within the conditions were too subtle. Second, the study relied on online participant recruitment and data collection. Online data collection was used for the purpose of generating a larger study sample; however, this benefit came with several costs. One was the method of recruitment and the participants comprising the sample. Study announcements were distributed through professors, organizational, and listserv connections - I had never personally met most of these connections I relied on. Due to my reliance on some secondary parties for 
distribution purposes, experimenter control was tenuous at best. Additionally, I verified participant enrollment in a BSN program, but I did not further investigate the program's academic requirements and recruitment efforts. It is possible that participants were at a school that placed a premium on male inclusion while others may not have been at such a school. The final limitation that I will touch on deals with the lack of an oral component. Prior stereotype threat research in the domain of communication has utilized audio recordings to code for tentative language as well as speech disfluencies (Breckinridge et al., 2011; McGlone \& Pfiester, in press). I was able to code for tentative language from the online simulation, but without an actual verbal recording, it was impossible to measure disfluency. It is also most likely that tentative language rates would have differed if the simulation had been audio recorded and not an online simulation due to participants' capacity to edit their written work.

Despite these limitations, the current study offers several intriguing paths for future investigations. One path should be to overcome the limitations addressed above. Specifically, researchers should seek to adjust the stereotype threat prime, data collection methodology (experimenter, in-person v. online, and utilization of a confederate as the patient), and the type of data collected during the experiment. First, future researchers might make the stereotype threat prime more direct and salient through the use of oral instructions (if conducted in-person) as well as more explicit written instructions. For example, an updated empathy prime could read as follows (update of the current priming paragraph is bolded):

The purpose of this research is to investigate differences in nurse's ability to empathize with patients. Empathic ability entails understanding a patient's circumstances and acting upon that understanding in order to make the patient 
feel understood, comfortable, and secure regarding the care being provided.

Empathic ability, which is essential to being an effective nurse, is commonly attributed more to women than men. Numerous scientific studies have demonstrated that empathic ability critically depends on communication behavior. This research has also found that empathic ability can be predicted from observing people as they simulate the communication behaviors they use when interacting with others. In today's study, you will simulate the interaction you would use when entering an examination room to explain the results of a medical test to a patient. Empathic individuals must be able to articulate necessary information clearly, so your performance in this simulation provides one way to measure your empathy level. Your simulation response will be analyzed later on by a team of researchers studying nurses' empathic ability here at the university. They will compare your recording to those of other men and women participating in this study to examine gender differences in performance.

Research supports the stance that empathy is essential to becoming an effective nurse (Reynolds \& Scott, 1999). This updated prompt is more overt in stating just that - to be a good nurse, one must be empathic. It also further strengthens the link between women and empathy, and highlights the distance between men and empathy. It is possible that male participants lacked the necessary identification with empathy (and female participants with patient management) in the current study or the prompts were too subtle to serve a purpose of eliciting threat.

Additionally, there is no guarantee that participants in this study actually read the pre-experiment prompt. Despite advancements in online methodology, it is not possible to determine where participants spent their time on the survey - only how long it took each participant to complete the study. Therefore, having the stereotype threat prime on paper as well as being articulated by an experimenter (if conducted in person) could improve the impact of the manipulation. Additionally, participants in the patient management and empathy conditions could be asked to complete a short-inventory related to their prime after reading the prompt - where the STAI-6 was incorporated in 
this study. For example, participants in the empathy frame could complete the Mehrabian and Epstein (1972) scale of emotional tendencies prior to the experimental simulation. Items in the scale include "It is hard for me to see how some things upset people so much" and "When a friend starts to talk bout his/her problems, I try to steer the conversation to something else." By responding to such items, it is possible that participants in the empathy frame would be more focused on their use of empathy and emotional availability. This type of objective data would help aid empathy-based subjective data collected during the experiment as both types of data hold the potential to generate differing results.

Second, by employing an in-person experimenter, steps can be taken to make sure participants use the entire five-minute preparation period and do not use notes during the simulation itself. Additionally, by adjusting the study methodology to in-person from online, it is possible to employ a confederate to serve as the patient in the experiment. As was addressed in the limitations section, it is likely that it was difficult for participants to imagine the patient - especially patient gender. Therefore, by having an actual patient in the room for the participant to interact with, patient gender will not have to be imagined as more social cues will be available to the participant - cues that are not available in online interactions (Dubrovsky, Kiesler, \& Sethna, 1991). Thus rendering the potential for different results if the study were to be conducted in person.

Finally from a methodological standpoint, future research should look to adjust the form of communication measured. In the current study, participants typed out a patient interaction simulation where the patient was nothing more than a prompt. Would we be able to notice a difference in results if there were an actual patient that interacted 
with the participant? This modification would also allow for participants to generate more natural oral responses to be audio-recorded rather than a more contrived written record. For this study, given that participants were not timed during the online simulation, they had the ability to edit their responses (even though directions instructed them not to do so). While participants could have edited their responses due to the methodology in place, if data were to be collected in person (orally), the ability to edit would not exist. Additionally, Graddy (2006) noted that men and women are comparable in their uses of tentative language online, a finding that is in contrast to research indicating women use more tentative language than men (e.g., Reid, Keerie, \& Palomares, 2003; Palomares, 2004, 2008). Therefore, the online component of this study may have leveled - not reduced or increased - the uses of tentative language by all participants. Thus, the possibility exists to observe mean differences in tentative language rates of male and female participants if data were to be collected in-person and not online. This method would also allow researchers to investigate other communicative cues, such as disfluencies.

In addition to proposing future directions that address this study's limitations, this project provides a framework for which scholars can expand their understanding of stereotype threat in interpersonal interactions. One of the first directions for future research should look to replicate this study with the use of practicing nurses and not nursing students as participants. Although the use of nursing students is a common substitution for research on nurses (Harding, 2008), students have a different relationship with and investment in the field of nursing than those who are practicing. It is also likely that practicing Registered Nurses (RNs) have more experience with gender-based 
stereotypes and/or have habituated to any threat they pose. Thus practicing RNs may be more resilient than are nursing students as they have learned how to deal with and react to the stressors associated with being a gendered minority (Coutu, 2002). Both explanations hold the capacity to alter the observed results on communicative performance in stereotype threat conditions.

It is also important to gain a greater understanding of the research participant's backgrounds. For example, participants were asked if they had a nurse in the family or if they had medical experience prior to enrolling in nursing school. However, they were not asked any questions about the recruitment of male nursing students at their respective academic institutions, support structures in place for male nursing students, experienced encouragement to pursue (or not to pursue) nursing from family and friends, and specific gender-based barriers they have had to overcome. This type of data would yield a much richer understanding of who is participating in the study. For example, male nursing students at the University of Texas at Austin have access to the Longhorn Association for Men in Nursing, a service that provides necessary resources and support to help men in the field of nursing. Participants who have experienced this type of socialization may be less susceptible to conditions of stereotype threat than participants who did not have a male nursing support group at their academic institution. Research may find that conditions of stereotype threat are only effective in particular contexts, with one of those being nurse socialization.

Additional future research might look to incorporate video-recordings and not just rely on audio-recordings (as previously proposed) during the simulation. A videorecording would afford researchers the opportunity to assess participants' nonverbal 
behaviors. For example, are participants more nonverbally constrained (i.e., reduced gesturing and facial expression) under conditions of threat? Given that nonverbals account for a significant portion of our understanding of human communication (Knapp \& Hall, 2009), the failure to collect such data renders findings from this type of stereotype threat research potentially incomplete. Finally, without the ability to observe nurses in their natural work environment, it is important to further our understanding of and the usefulness of imagined interaction theory (Honeycutt 2003, 2008, 2010). Does it matter that the research participant knows the patient is "not real" and therefore they do not take the experiment as serious as they could? While there is ample research that supports the use of imagined interactions, these issues ought to be further addressed in future research.

As the number of men entering the field of nursing continues to grow, it is important to identify the gender-based barriers and stereotypes faced by male nurses as to increase the quality of patient care and reduce nurse-attrition rates. It is also crucial to gain a greater comprehension of what attracts men to nursing and what keeps them away. This level of understanding would allow academic institutions, professional recruiters, and researchers alike to be able to actively recruit men more efficiently into the field to address the current nursing shortage, while also working to reduce attrition rates. Such efforts are necessary as the nursing workforce is highly homogenous with Caucasian females (D'Antonio \& Whelan, 2009; O'Lynn, 2004) providing care for a more genderbalanced and ethnically diverse population. Findings from this study also begin to shed light on the influence subtle stereotyping has on the communicative abilities of nurses. Specifically, it does not appear as though stereotype threat holds the same disruptive 
potential in imagined interpersonal interactions as has been previously reported in actual interpersonal interactions (e.g., McGlone \& Pfiester, in press). Moreover, it is important to determine if the potential effects of stereotype threat in the nurse-patient relationship is present in actual interpersonal interactions and not just imagined ones. Additionally, male participants identified more strongly with feminine behavioral norms, yet this does not provide an explanation for the lack of gender-neutral and gender-male examples in nursing texts, lectures, and recruitment materials. Just because a man identifies with feminine norms does not mean that he wants to be considered feminine. Finally, findings point to patient gender being more influential to the nurse-patient relationship than individual attributes (e.g., empathy). Thus, opportunities to develop training materials exist where recommendations on how to interact with patients based on their gender could be offered. In summary, this dissertation helps to further the study of stereotype threat in the field of communication - especially when it comes to the use of tentative language in patient-interaction simulations. Based on this research, we now have a better understanding of the gender-based biases and stereotypes faced by male nurses. These findings can be used to provide support for male nurses so that they may excel in interpersonal health contexts. 


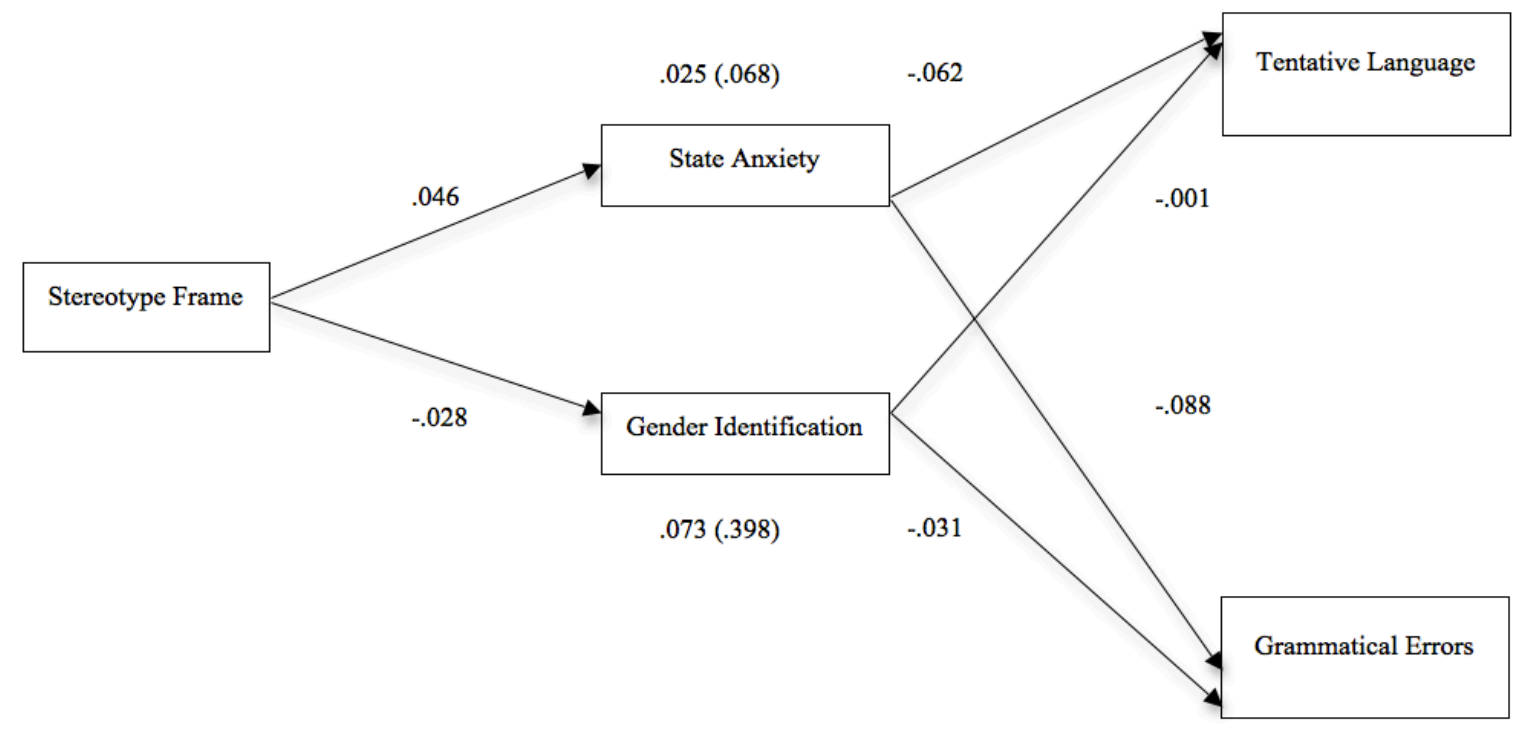

Figure 1. Multiple mediation analysis results. (Indirect Effects in Parentheses) 


\section{Appendix A: Tables}

Table 1

Demographic profile of the study sample

\begin{tabular}{|c|c|c|c|}
\hline Demographic Variable & Frequency & Percentage & \\
\hline $\begin{array}{l}\text { Gender } \\
\text { Male } \\
\text { Female }\end{array}$ & $\begin{array}{l}183 \\
192\end{array}$ & $\begin{array}{l}48.8 \% \\
51.2 \%\end{array}$ & \\
\hline $\begin{array}{l}\text { Race/Ethnicity } \\
\text { African American/Black } \\
\text { American Indian } \\
\text { Asian/Pacific Islander } \\
\text { Hispanic/Latino(a) } \\
\text { Middle-Eastern } \\
\text { Multi-Racial } \\
\text { White/Caucasian } \\
\text { Other }\end{array}$ & $\begin{array}{l}42 \\
12 \\
25 \\
17 \\
2 \\
20 \\
223 \\
10\end{array}$ & $\begin{array}{l}11.2 \% \\
3.2 \% \\
6.7 \% \\
4.5 \% \\
.5 \% \\
5.3 \% \\
59.5 \% \\
2.7 \%\end{array}$ & \\
\hline $\begin{array}{l}\text { Regional Location } \\
\text { Northeast } \\
\text { Midwest } \\
\text { South } \\
\text { West }\end{array}$ & $\begin{array}{l}92 \\
88 \\
112 \\
61\end{array}$ & $\begin{array}{l}24.5 \% \\
23.5 \% \\
29.9 \% \\
16.3 \%\end{array}$ & \\
\hline $\begin{array}{l}\text { Nurse in Immediate Family } \\
\text { Yes } \\
\text { No }\end{array}$ & $\begin{array}{l}78 \\
283\end{array}$ & $\begin{array}{l}20.8 \% \\
75.5 \%\end{array}$ & \\
\hline $\begin{array}{l}\text { Prior Experience in Medical Field } \\
\text { Yes } \\
\text { No }\end{array}$ & $\begin{array}{l}105 \\
270\end{array}$ & $\begin{array}{l}28.0 \% \\
72.0 \%\end{array}$ & \\
\hline & Range & Mean & SD \\
\hline Age & 18-49 Years & 24.97 Years & 5.76 Years \\
\hline Time as a Nursing Student & 1-76 Months & 12.42 Months & $\begin{array}{l}10.06 \\
\text { Months }\end{array}$ \\
\hline
\end{tabular}


Table 2

Histogram of the Gender-Related Work Barriers Inventory

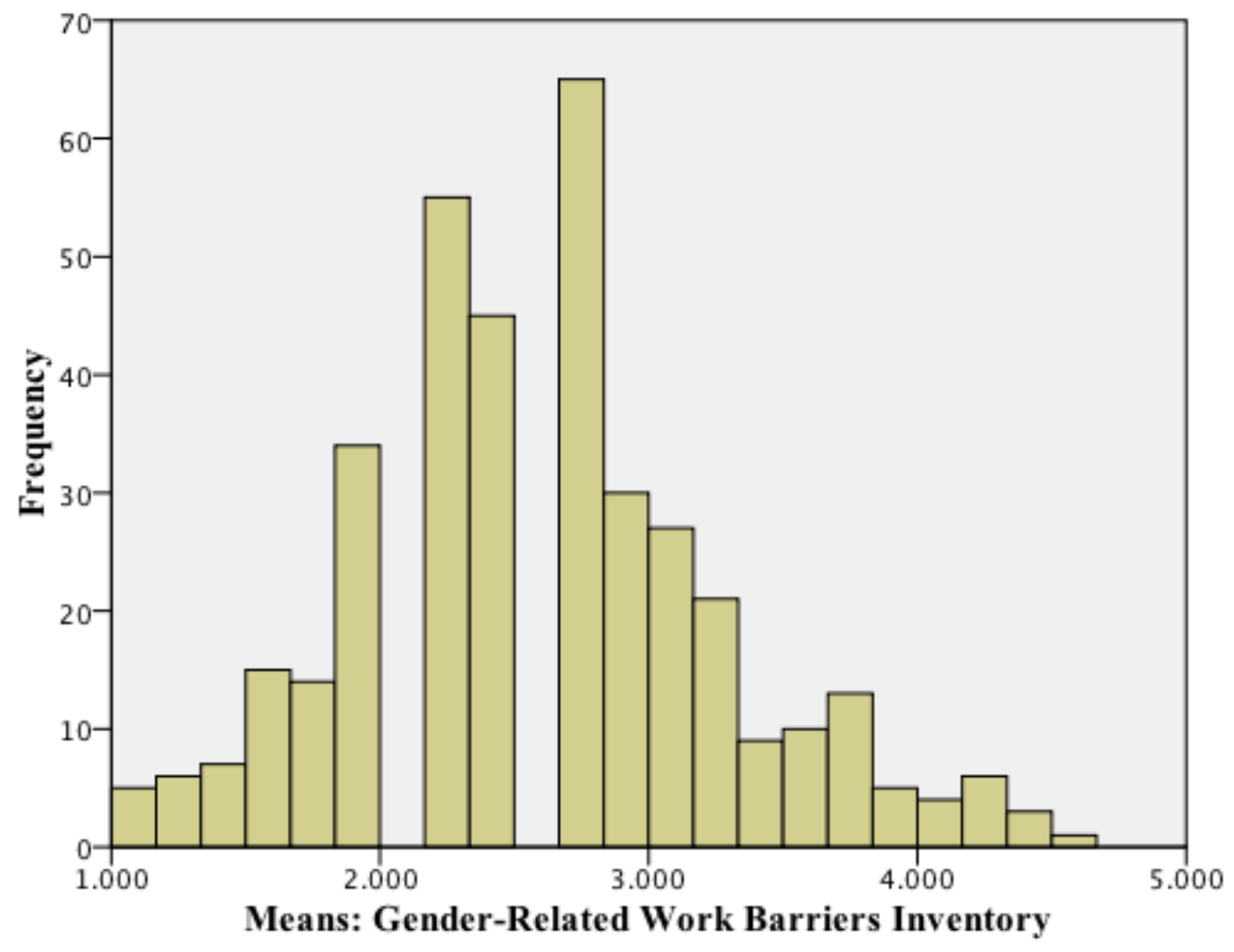


Table 3

Histogram of the Gender-Related Work Barriers Inventory (Male Participants)

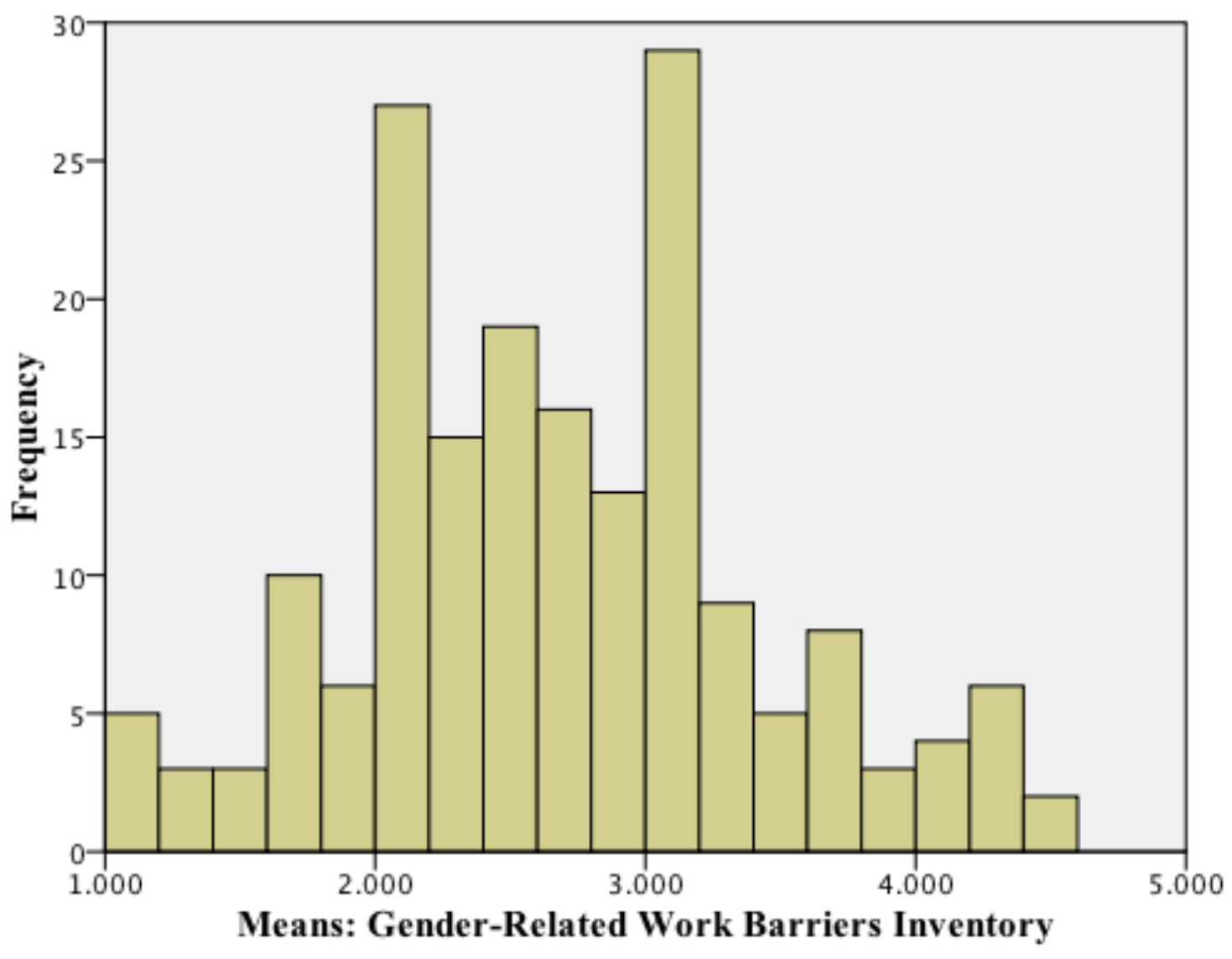


Table 4

Histogram of the Gender-Related Work Barriers Inventory (Female Participants)

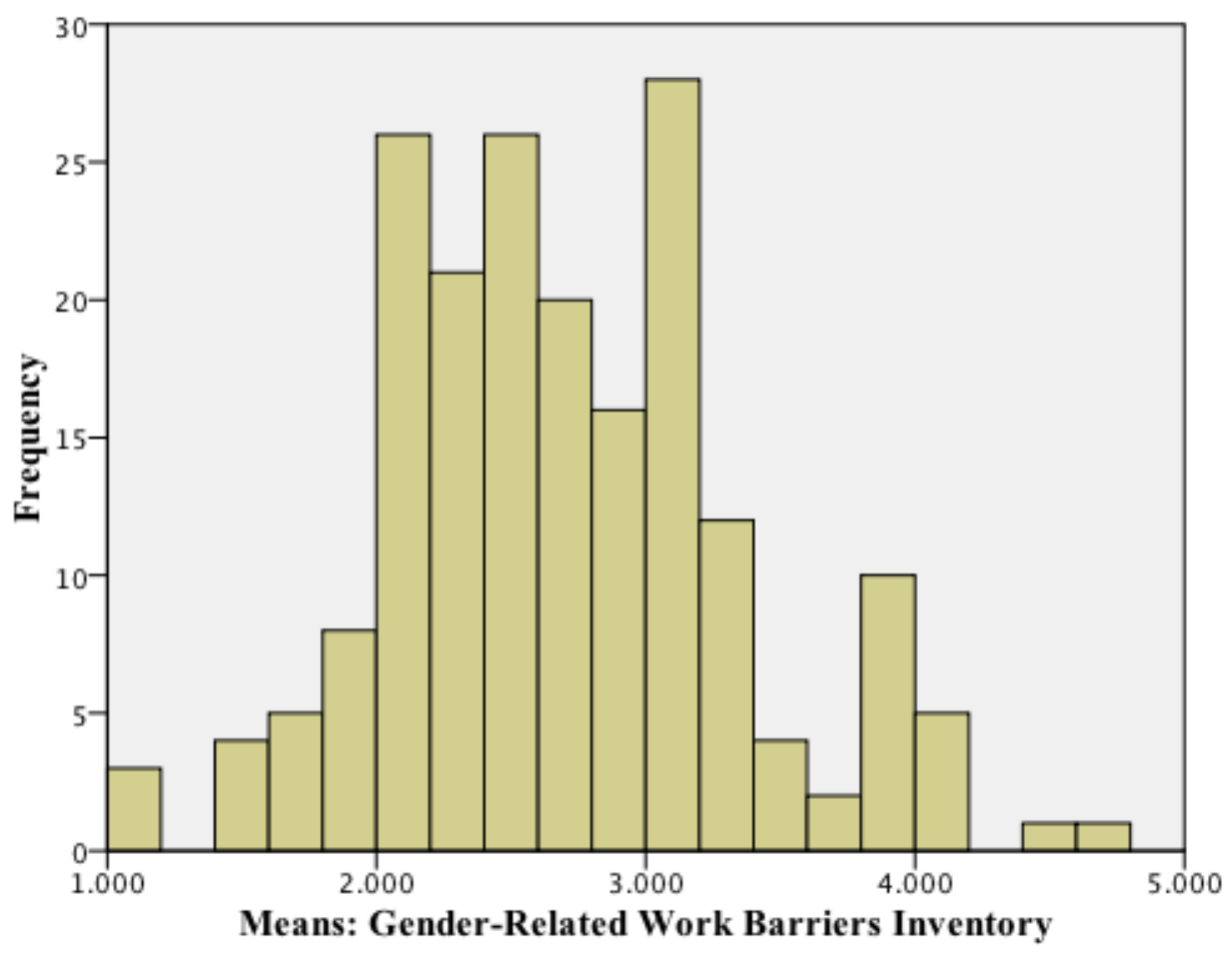


Table 5

Participant Cell Distribution Across Participant Gender, Patient Gender, and Diagnostic

Frame

\begin{tabular}{|c|c|c|c|c|c|}
\hline & & & Patien & nder & \\
\hline Particip & at Gender & & Male & Female & Total \\
\hline Male & Diagnostic & Patient & 31 & 30 & 61 \\
\hline & Frame & Management & & & \\
\hline & & Empathy & 30 & 31 & 61 \\
\hline & & Control & 30 & 31 & 61 \\
\hline & Total & & 91 & 92 & 183 \\
\hline Female & Diagnostic & Patient & 31 & 31 & 62 \\
\hline & Frame & Management & & & \\
\hline & & Empathy & 33 & 31 & 64 \\
\hline & & Control & 36 & 30 & 66 \\
\hline & Total & & 100 & 92 & 192 \\
\hline Total & Diagnostic & Patient & 62 & 61 & 123 \\
\hline & Frame & Management & & & \\
\hline & & Empathy & 63 & 62 & 125 \\
\hline & & Control & 66 & 61 & 127 \\
\hline & Total & & 191 & 184 & 375 \\
\hline
\end{tabular}


Table 6

Mean (SD) Rates of Tentative Language and Grammatical Errors by Participant Gender, Patient Gender, and Diagnostic Frame

\section{Diagnostic Frame}

\begin{tabular}{cccc} 
Dependent Measure & $\begin{array}{c}\text { Patient } \\
\text { Management }\end{array}$ & Empathy & Control \\
\hline Tentative Language Rate* & & & \\
Male: MP & $4.61(2.94)$ & $6.00(4.27)$ & $4.60(2.91)$ \\
FP & $5.23(2.36)$ & $4.13(2.50)$ & $4.71(2.65)$ \\
Female: MP & $5.68(3.33)$ & $4.76(3.00)$ & $4.94(2.53)$ \\
FP & $5.71(2.96)$ & $5.81(3.61)$ & $5.80(3.63)$
\end{tabular}

Grammatical Errors*

\begin{tabular}{|c|c|c|c|c|}
\hline \multirow[t]{2}{*}{ Male: } & MP & $5.57(3.42)$ & $6.43(4.47)$ & $6.48(4.79)$ \\
\hline & $\mathrm{FP}$ & $4.81(2.91)$ & $5.79(4.54)$ & $8.76(6.61)$ \\
\hline Female: & MP & $6.13(4.96)$ & $7.31(4.85)$ & $5.08(2.94)$ \\
\hline & $\mathrm{FP}$ & $6.74(4.47)$ & $5.57(3.95)$ & $5.90(4.04)$ \\
\hline
\end{tabular}

* per 100 words MP = Male Patient; FP = Female Patient 
Table 7

Mean (SD) Rates of Tentative Language and Grammatical Errors by Patient Gender for Male Participants (Post Hoc Analysis)

\begin{tabular}{llll}
\hline Dependent Measure & Male Patient & Female Patient & Total \\
\hline Tentative Language & $4.68(2.52)$ & $5.07(3.45)$ & $4.87(3.02)$ \\
Grammatical Errors & $6.47(5.17)$ & $6.15(4.23)$ & $6.31(4.72)$ \\
\hline
\end{tabular}


Table 8

Mean (SD) Rates of Tentative Language and Grammatical Errors by Patient Gender for Female Participants (Post Hoc Analysis)

\begin{tabular}{llll}
\hline Dependent Measure & Male Patient & Female Patient & Total \\
\hline Tentative Language & $5.77(3.37)$ & $5.11(2.95)$ & $5.43(3.17)$ \\
Grammatical Errors & $6.07(4.15)$ & $6.13(4.36)$ & $6.10(4.25)$ \\
\hline
\end{tabular}


Table 9

Correlation Matrix of GRWB, CMNI, \& CFNI

\begin{tabular}{|c|c|c|c|c|}
\hline & Mean (SD) & GRWB & CMNI & CFNI \\
\hline GRWB & $2.67(.71)$ & 1 & .093 & $-.143^{* *}$ \\
\hline CMNI & $2.23(.28)$ & .093 & 1 & $-.337^{* *}$ \\
\hline CFNI & $2.87(.31)$ & $-.143^{* *}$ & $-.337^{* *}$ & 1 \\
\hline
\end{tabular}

** Correlation is significant at the 0.01 level (2-tailed) 


\section{Appendix B: IRB Approval}

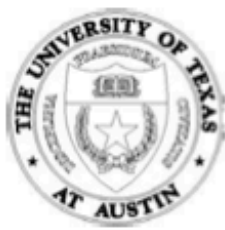

OFFICE OF RESEARCH SUPPORT

THE UNIVERSITY OF TEXAS AT AUSTIN

P.O. Box 7426, Austin, Texas $78713 \cdot$ Mail Code $A 3200$

(512) 471-8871 $\cdot F A X(512) 471-8873$

FWA \# 00002030

Date: $05 / 23 / 12$

PI: $\quad$ Matthew S McGlone

Dept: Communication Studies

Title: Communication in the Health Setting

Re: IRB Exempt Determination for Protocol Number 2012-02-0106

Dear Matthew S McGlone:

Recognition of Exempt status based on 45 CFR 46.101(b)(2).

Qualifying Period: $05 / 23 / 2012$ to $05 / 22 / 2015$. Expires 12 a.m. [midnight] of this date. A continuing review report must be submitted in three years if the research is ongoing.

\section{Responsibilities of the Principal Investigator:}

Research that is determined to be Exempt from Institutional Review Board (IRB) review is not exempt from ensuring protection of human subjects. The following criteria to protect human subjects must be met. The Principal Investigator (PI):

1. Assures that all investigators and co-principal investigators are trained in the ethical principles, relevant federal regulations, and institutional policies governing human subject research.

2. Will provide subjects with pertinent information (e.g., risks and benefits, contact information for investigators and IRB Chair) and ensures that human subjects will voluntarily consent to participate in the research when appropriate (e.g., surveys, interviews).

3. Assures the subjects will be selected equitably, so that the risks and benefits of the research are justly distributed.

4. Assures that the IRB will be immediately informed of any information or unanticipated problems that may increase the risk to the subjects and cause the category of review to be reclassified to expedited or full board review.

5. Assures that the IRB will be immediately informed of any complaints from subjects regarding their risks and benefits. 
Re: IRB Exempt Determination for Protocol Number 2012-02-0106

Page 2 of 2

6. Assures that confidentiality and privacy of the subjects and the research data will be maintained appropriately to ensure minimal risks to subjects.

7. Will report, by amendment, any changes in the research study that alter the level of risk to subjects.

These criteria are specified in the PI Assurance Statement that was signed before determination of exempt status was granted. The PI's signature acknowledges that they understand and accept these conditions. Refer to the Office of Research Support (ORS) website www.utexas.edu/irb for specific information on training, voluntary informed consent, privacy, and how to notify the IRB of unanticipated problems.

1. Closure: Upon completion of the research study, a Closure Report must be submitted to the ORS.

2. Unanticipated Problems: Any unanticipated problems or complaints must be reported to the IRB/ORS immediately. Further information concerning unanticipated problems can be found in the IRB Policies and Procedure Manual.

3. Continuing Review: A Continuing Review Report must be submitted if the study will continue beyond the three year qualifying period.

4. Amendments: Modifications that affect the exempt category or the criteria for exempt determination must be submitted as an amendment. Investigators are strongly encouraged to contact the IRB Program Coordinator(s) to describe any changes prior to submitting an amendment. The IRB Program Coordinator(s) can help investigators determine if a formal amendment is necessary or if the modification does not require a formal amendment process.

If you have any questions contact the ORS by phone at (512) $471-8871$ or via e-mail at orsc@uts.cc.utexas.edu.

Sincerely,

tams $P \omega=f$ son

http://www.utexas.edu/irb

James Wilson, Ph.D.

Institutional Review Board Chair 


\title{
Appendix C: Consent Form
}

\author{
Communication in the Health Setting
}

IRB \# 2012-02-0106

Conducted by: Andrew C. Tollison, M.S. Investigator; atollison@utexas.edu; 512-471-5251

Matt McGlone, Ph.D., Faculty Sponsor; matthew_mcglone@mail.utexas.edu; 512-471-1920

Dept. of Communication Studies, The University of Texas at Austin

You are being asked to participate in a research study. This form provides you with information about the study. The person in charge of the research will also describe the study to you and answer all of your questions. Please read the information below and ask questions about anything you do not understand before deciding whether or not to take part. Your participation is entirely voluntary and you can refuse to participate without penalty or loss of benefits to which you are otherwise entitled. You may stop participating at any time by simply telling the researcher.

The purpose of this research is to investigate gender difference in communication abilities of healthcare associates.

If you agree to be in the study, you are asked to do the following things:

Review a short scenario

Participate in a 2-minute nurse-patient interaction simulation

Fill out a 5-part questionnaire

Total estimated time to participate in this study is 25 minutes.

Risks and benefits of being in the study:

The risks of participating in the study are no greater than those of everyday life.

There are no individual benefits to participating in this study, however, you will be helping researchers understand the extent to which gender differences exist in healthcare communication.

\section{Compensation:}

- You will have the opportunity to win one of ten $\$ 50$ gift cards.

The records of this study will be stored securely and kept private. Authorized persons from the University of Texas at Austin and members of the Institutional Review Board have the legal right to review your research records and will protect the confidentiality of those records to the extent permitted by law. All publications will exclude any information that will make it possible to identify you as a subject. Your name will not be connected in any way with your completed surveys.

\section{Contacts and questions:}

If you have any questions, want additional information, or wish to withdraw your 
participation, please contact Andrew Tollison. His phone number and email address are at the top of this page.

If you have questions about your rights as a research participant, complaints, concerns, or questions about the research please contact Jody Jensen, Ph.D., Chair, The University of Texas at Austin Institutional Review Board for the Protection of Human Subjects at 512232-2685 or the Office of Research Support and Compliance at 512-471-8871 or email: orsc@uts.cc.utexas.edu.

Please print a copy of this form to keep for your records. By selecting "Next," you agree to participate in this study. 


\section{Appendix D: Participant Recruitment Letter}

Title: Communication in the Health Setting

Researcher: Andy Tollison

You are being asked to participate in a research study. The purpose of this research is to investigate the extent to which gender differences influence the communication abilities of individuals working in healthcare. Approximately 360 people will participate in this study, and anyone of at least 18 years of age who is enrolled in a BSN program is eligible. You will be asked to review a short medical scenario, participate in a 3-minute procedural simulation, and fill out a short survey. All of which will take approximately 30 minutes to complete. You can participate anytime online using the following URL:

Your participation is completely voluntary and you will have the opportunity to enter a raffle to win one of ten $\$ 50$ gift cards for participating. Thank you in advance for your participation - it is greatly appreciated!

Please contact Andy Tollison at atollison@utexas.edu if you have any questions. 


\title{
Appendix E: Representative Simulation Prompt
}

\author{
Patient: Amy Jones
}

\section{Please review the following paragraph:}

The purpose of this research is to investigate differences in nurses' abilities to manage patients. Patient management ability entails understanding a patient's circumstances and acting upon that understanding in order to make the patient feel understood, comfortable, and secure regarding the care being provided. Numerous scientific studies have demonstrated that patient management ability can be predicted from observing people as they simulate the communication behaviors they use when interacting with others. In today's study, you will simulate the interaction you would use when entering an examination room to explain the results of a medical test to a patient. Patient managers must be able to articulate necessary information clearly, so your performance on this simulation provides one way to measure your patient management ability in the medical field. Your simulation response will be analyzed later on by a team of patient management researchers here at the university. They will compare your responses to those of other men and women participating in this study to examine gender differences in performance.

\section{Now, please review the following scenario:}

Today you will be assisting Dr. Smith with patient Amy Jones. Amy came to the physician's office today because she was not feeling well. Dr. Smith has diagnosed her with the stomach flu. Due to an emergency in the office, Dr. Smith has asked you to inform the patient of their diagnosis as well as instruct the patient on their prescription (including the name of the medication, medication instructions, and side effects). Dr. Smith will stop back by later to address any questions patient Jones may have before they leave.

\section{Here is a breakdown of the information you will need to give to patient Jones:}

Diagnosis: Influenza or stomach flu

\section{Medication: TAMIFLU®}

Medication Instructions: Take orally one (1) capsule (75 mg) twice (2) daily for five (5) days. TAMIFLU ${ }$ can be taken with or without food, however, there is less chance of upsetting the patient's stomach if the TAMIFLU ${ }$ is taken with a light snack, milk, or a meal.

Side Effects: The most common side effects of TAMIFLU® are nausea and vomiting. These are usually mild to moderate. Side effects usually happen in the first two days of treatment. Taking TAMIFLUß with food may reduce the chance of getting these effects. If you develop an allergic reaction or a severe rash, stop taking TAMIFLU ${ }^{\circledR}$ immediately and contact our office. 


\section{Appendix F: AIDET Framework}

As you complete the simulation, we ask that you use the AIDET framework, which helps nurses communicate more effectively with patients. Be as specific as possible as you relay the necessary information to the patient. Research has demonstrated that using AIDET when interacting with patients increases that patient's satisfaction with their medical experience. Here is a breakdown of the AIDET framework that you will use during the simulation:

Acknowledge: Greet the patient as if you were just entering the room and use the patient's name.

Introduce: Introduce yourself to the patient politely. Tell them who you are and how you will be assisting them today.

Duration: Your goal is to ease the patient's mind as they wait for Dr. Smith to return.

Explanation: Advise the patient about their diagnosis and medication instructions.

Thank You: Thank the patient for their patronage and patience. 


\section{Appendix G: Personal Report of Communication Apprehension (McCroskey, 1982)}

Directions: This instrument is composed of twenty-four (24) statements concerning feelings about communicating with other people. Please indicate the degree to which each statement applies to you by circling whether you: Strongly Agree (1), Agree (2), Undecided (3), Disagree (4), or Strongly Disagree (5).

Work quickly and record your first impression.

1. I dislike participating in group discussion

2. Generally, I am comfortable while participating in group discussions.

3. I am tense and nervous while participating in group discussions.

4. I like to get involved in group discussions.

5. Engaging in group discussion with new people makes me tense and nervous.

6. I am calm and relaxed while participating in group discussions.

7. Generally, I am nervous when I participate in a meeting.

8. Usually I am calm and relaxed while participating in meetings.

9. I am very calm and relaxed when I am called upon to express an opinion at a meeting.

10. I am afraid to express myself at meetings.

11. Communicating at meetings usually makes me uncomfortable.

12. I am very relaxed when answering questions at a meeting.

13. While participating in a conversation with a new acquaintance, I feel very nervous.

14. I have no fear of speaking up in conversations.

15. Ordinarily I am very tense and nervous in conversations.

16. Ordinarily I am very calm and relaxed in conversations.

17. While conversing with a new acquaintance, I feel very relaxed.

18. I'm afraid to speak up in conversations.

19. I have no fear of giving a speech.

20. Certain parts of my body feel very tense and rigid while giving a speech. 
21. I feel relaxed while giving a speech.

22. My thoughts become confused and jumbled when I am giving a speech.

23. I face the prospect of giving a speech with confidence.

24. While giving a speech, I get so nervous I forget facts I really know. 


\section{Appendix H: State-Trait Anxiety Inventory (Marteau \& Bekker, 1992)}

Directions: You will have 1 minute to complete the following six items. Do not think about the items in relation to your everyday life; just identify how you currently feel. Please use the identified scale for your responses. Circle the appropriate response for each item.

\begin{tabular}{|c|c|c|c|c|}
\hline & Not at all & Somewhat & Moderately & Very much \\
\hline 1. I feel calm. & 1 & 2 & 3 & 4 \\
\hline 2. I am tense. & 1 & 2 & 3 & 4 \\
\hline 3. I feel upset. & 1 & 2 & 3 & 4 \\
\hline 4. I am relaxed. & 1 & 2 & 3 & 4 \\
\hline 5. I feel confident. & 1 & 2 & 3 & 4 \\
\hline 6. I am worried. & 1 & 2 & 3 & 4 \\
\hline
\end{tabular}




\section{Appendix I: Conformity to Masculine \& Feminine Norms Inventory (Parent \& Moradi, 2009; 2010)}

The following pages contain a series of statements about how people might think, feel or behave. The statements are designed to measure attitudes, beliefs, and behaviors associated with both traditional and non-traditional masculine and feminine gender roles.

Thinking about your own actions, feelings and beliefs, please indicate how much you personally agree or disagree with each statement by indicating whether you Strongly Disagree (SD), Disagree (D), Agree (A), or Strongly Agree (SA) to the right of each statement.

1. In general, I will do anything to win

2. If I could, I would frequently change sexual partners

3. I would only be satisfied with sex if there was an emotional bond

4. I believe that violence is never justified

5. Being thought of as gay is not a bad thing

6. In general, I do not like risky situations

7. Winning is not my first priority

8. I enjoy taking risks

9. I am disgusted by any kind of violence

10. I ask for help when I need it

11. My work is the most important part of my life

12. I would only have sex if I was in a committed relationship

13. I bring up my feelings when talking to others

14. I would be furious if someone thought I was gay

15. I don't mind losing

16. I take risks

17. It would not bother me at all if someone thought I was gay

18. I never share my feelings

19. Sometimes violent action is necessary

20. In general, I control the women in my life

21. I would feel good if I had many sexual partners

22. It is important for me to win

23. I don't like giving all my attention to work

24. It would be awful if people thought I was gay

25. I like to talk about my feelings

26. I never ask for help

27. More often than not, losing does not bother me

28. I frequently put myself in risky situations

29. Women should be subservient to men

30. I am willing to get into a physical fight if necessary

31. I feel good when work is my first priority

32. I tend to keep my feelings to myself

33. Winning is not important to me

34. Violence is almost never justified

35. I am happiest when I'm risking danger

36. It would be enjoyable to date more than one person at a time

37. I would feel uncomfortable if someone thought I was gay 
38. I am not ashamed to ask for help

39. Work comes first

40. I tend to share my feelings

41. No matter what the situation I would never act violently

42. Things tend to be better when men are in charge

43. It bothers me when I have to ask for help

44. I love it when men are in charge of women

45. I hate it when people ask me to talk about my feelings

46. I try to avoid being perceived as gay

47. I would be happier if I was thinner

48. It is important to keep your living space clean

49. I tell everyone about my accomplishments

50. I clean my home on a regular basis

51. I believe that my friendships should be maintained at all costs

52. I find children annoying

53. I would feel guilty if I had a one-night stand

54. When I succeed, I tell my friends about it

55. Having a romantic relationship is essential in life

56. I enjoy spending time making my living space look nice

57. Being nice to others is extremely important

58. I don't go out of my way to keep in touch with friends

59. Most people enjoy children more than I do

60. I would like to lose a few pounds

61. It is not necessary to be in a committed relationship to have sex

62. I hate telling people about my accomplishments

63. I would feel burdened if I had to maintain a lot of friendships

64. I would feel comfortable having casual sex

65. I make it a point to get together with my friends regularly

66. I always downplay my achievements

67. Being in a romantic relationship is important

68. I don't care if my living space looks messy

69. I always try to make people feel special

70. I am not afraid to tell people about my achievements

71. My life plans do not rely on my having a romantic relationship

72. I am always trying to lose weight

73. I would only have sex with the person I love

74. When I have a romantic relationship, I enjoy focusing my energies on it

75. There is no point to cleaning because things will get dirty again

76. I am not afraid to hurt people's feelings to get what I want

77. Taking care of children is extremely fulfilling

78. I would be perfectly happy with myself even if I gained weight

79. If I were single, my life would be complete without a partner

80 . I rarely go out of my way to act nice

81. I actively avoid children

82. I am terrified of gaining weight

83. I would only have sex if I was in a committed relationship like marriage

84. I like being around children

85 . I don't feel guilty if I lose contact with a friend

86 . I would be ashamed if someone thought I was mean 


\section{Appendix J: Nursing Identification Measure (Mael \& Ashforth, 1992; Modified)}

Directions: Think of your role as a nursing student. For each item below, select the response that best represents your belief about or attitude toward nursing. Use the following response scale: $\mathbf{1}$ (Strongly Agree), 2 (Agree), 3 (Somewhat Agree), 4 (Neither Agree nor Disagree), 5

(Somewhat Disagree), 6 (Disagree), or 7 (Strongly Disagree)

1. In general, when someone praises nurses, it feels like a personal compliment.

2. In general, when someone criticizes nurses, it feels like a personal insult.

3. When I talk about nurses, I usually say "we" rather than "they."

4. Medicine's successes are my successes.

5. If a story in the media criticizes nurses, I would feel embarrassed.

6. I am very interested in what others think about nurses. 


\section{Appendix K: Gender-Related Work Barriers Measure (Rochlen, Good, \& Carver, 2009)}

Directions: Think of your role as a nursing student. For each item below, select the answer that best represents your belief about or attitude toward nursing. Use the following response scale: 1 (Strongly Disagree), 2 (Disagree), 3 (Neither Agree nor Disagree), 4 (Agree), or 5 (Strongly Agree).

1. Given the large number of women in the nursing field, I sometimes feel like an outsider.

2. As a male nurse, I experience obstacles that may not be present for women.

3. As a male nurse, I've never thought much about how I may be experienced differently by patients.

4. Male and female nurses are treated similarly by others.

5. Being a male nurse negatively affects my personal life outside the classroom.

6. My gender (i.e., being male) is not an issue in the workplace. 


\section{Appendix L: Demographics Measure}

\section{Please tell us a little about yourself.}

1. How old are you? \# of years

2. Gender: Male Female

3. I identify with this racial/ ethnic group (you may choose more than one):

African American/ Black American Indian Asian/ Pacific Islander Hispanic/ Latino(a)

4. I have been a nursing student for Middle Eastern Multi-Racial White/ Caucasian Other (please specify:

5. What specialization of nursing are you studying?

6. What region of the United States are you attending school in?

Northeast

Midwest

South

West

7. Did you have any medical experience before being admitted to the nursing program?

$$
\text { Yes No }
$$

7a. If yes, what type of prior medical experience did you have?

8 . Is there a nurse in your immediate family?

8a. If so, what is your relationship to that individual? 


\section{Appendix M: Tentative Language Codebook}

Directions: Each of you will initially be assigned 94 cases to review. The cases you will be reviewing are from an online simulation where a nurse (the participant in this study) is entering a patient's room to tell them they have the flu. One of the variables in the current study is the use of tentative language by the research participant.

The cases you will be reviewing are verbatim from the participant so there are grammatical errors. As you go through the cases, I want you to code for five criteria. You will be assigning an overall frequency score for each of the categories (greater detail of each category is provided on the following pages):
1. Disclaimers
2. Hedges
3. Tag Questions
4. Intensifiers
5. Rounders

\section{Tentative Language Categories}

\section{1) Tally the number of DISCLAIMERS}

Disclaimers typically PRECEDE a statement or opinion (e.g., I'm not an expert...). However, this is not always the case. The examples below provide an overview of what to look for when attempting to identify the disclaimers that appear in the cases you review. This list is not all encompassing.

- $\quad$ I think

- I believe

- Seems to be

- It seems that

- It seems like

- It suggests that

- I may be wrong

- I'm not sure

- I mean

- I suppose

- I'm no expert

- It appears that

- It may be the case that

- It points to

- One might speculate that

- One is struck by

- It is (un)likely that... 
- I don't know

- I'm not sure

- As far as I can tell

- According to

- I am hoping

- The assumption

- I hope

- I thought

- If I remember correctly

- Please correct me if I'm wrong

- At least the (quote) said

- It sounds like you...

- You may find that...

- I doubt it

2) Tally the number of HEDGES

Hedges are adverbs/phrases used in almost always the middle of a sentence to weaken or strengthen the current statement (e.g., The flu is kind of like...). However, these statements can show up at other parts of a sentence and not just in the middle - although they frequently occur in the middle. The examples below provide an overview of what to look for when attempting to identify the hedges that appear in the cases you review. This list is not all encompassing.

- Probably

- Pretty much

- Y'know

- You know

- Kinda

- $\quad$ I think

- I guess

- Sort of

- Perhaps

- Maybe

- May

- Like

- Kind of

- Whatever

- ...might be

- Somewhat

- Something

- Modification of term by adding the morpheme -y (e.g., We all agree it was a thingy on his neck?" 
- Technically

- Little bit

- Approximately

- A bit (e.g., It is a bit cold in here)

- I suppose

- Almost

- Perhaps

- One (can imagine)

- Even though

- Possibly

- Might

- Unless

- Basically

- It could be

- Around

- About

- In some ways

- A few

- Relatively

- In a way

- Reportedly

- Could

- Some of the

- More or less

- Any

- Anybod*

- Anything

- Appear

- Appears

- Bet

- Chance

- Doubt

- Fairly

- Generally

- Hesita*

- Hope

- Hopefully

- Hopes

- If

- Likel*

- Lot

- Lots

- Lucki*

- Mainly

- May 
- Maybe

- Might

- Most

- Mostly

- Often

- Or

- Perhaps

- Possib*

- Pretty

- Probably

- Question

- Quite

- Seem

- Seems

- Some

- Something*

- Sometimes

- Somewhat

- Sort

- Suppose

- Supposed

- Tempora*

- Typically

- Unlikel*

- Usually

- Varies

\section{3) Tally the number of TAG QUESTIONS}

Tag questions follow declarative statements (e.g., Tamiflu is a medication that will help you illness, alright?). The examples below provide an overview of what to look for when attempting to identify the tag questions that appear in the cases you review. This list is not all encompassing.

- Isn't it?

- Doesn't it?

- You know?

- Right?

- Don't you think?

- Don't they?

- Aren't they?

- Wouldn't you say?

- Can you? 
- Didn't?

- Would you

4) Tally the number of INTENSIFIERS

Intensifiers are adverbs that are used to provide emphasis or intensity to a statement (e.g., This medicine is really important to take). The examples below provide an overview of what to look for when attempting to identify the intesifiers that appear in the cases you review. This list is not all encompassing.

- $\quad$ So

- Vastly

- Awfully

- Very

- Really

- Truly

- Extremely

- Certainly (do)

- Must

- Horrendous

- Still

- Never

- Obviously

- Definitely

- The fact that

- The truth of the matter is

- We are positive

5) Tally the number of ROUNDERS

Rounders represent a range of values where greater precision is either unavailable or irrelevant (e.g., You'll feel better in about $5-7$ days). The examples below provide an overview of what to look for when attempting to identify the rounders that appear in the cases you review. This list is not all encompassing.

- Less than a centimeter

- From 5-7 days

- About...

- ...or two 


\section{Appendix N: Grammatical Error Codebook}

Directions: Each of you will initially be assigned 94 cases to review. The cases you will be reviewing are from an online simulation where a nurse (the participant in this study) is entering a patient's room to tell them they have the flu. One of the variables in the current study is the grammatical correctness of the information that is being delivered to the patient.

The cases you will be reviewing are verbatim from the participant. I would like for you to review each assigned case and identify each grammatical error by drawing a line in the transcript where the error occurs. Additionally, I would like for you to tally the overall amount of grammatical errors per participant and input that into the box to the right of the case. You will see the use of medical terminology in the cases and these are not exempt from your review. The medical terms you will see include flu, influenza, and Tamiflu (these can be presented in upper case or lower case form). If you have a question about a presented medical term, please contact me. After you finish coding the initial 94 cases, we will divide up the remainder cases for your review. 


\section{Appendix O: Empathy Codebook}

Directions: Each of you will initially be assigned 94 cases to review. The cases you will be reviewing are from an online simulation where a nurse (the participant in this study) is entering a patient's room to tell them they have the flu. One of the variables in the current study is the degree to which each participant has demonstrated a degree of empathy towards their patient. For purposes of your review, empathy is defined as: "the ability to understand the patient's situation, perspective and feelings, and to communicate that understanding to the patient" (Coulehan et al., 2001).

The cases you will are verbatim from the participant so there are grammatical errors. As you go through your list, I want you to take them at face value. After reading each case, assign it a score from 1-3 based on the degree of empathy the person has demonstrated in their statement. Below you will see an outline and sample case for each of the scores. After you finish coding the initial 94 cases, we will divide up the remainder cases for your review.

1= Least empathic: Cases receiving a " 1 " merely give the facts to the patient with no attempt to show concern for the patient. The focus here is on revealing information about the medical situation only.

Case 81: Hi. I'm your nurse today. Dr. Smith asked me to come speak with you while he takes care of another patient. Based on the tests he ran and your symptoms, he's pretty sure you have the flu. Go ahead and take this medicine, TAMIFLU, and you shouldn't have any symptoms after a week. Is there anything I can answer for you? Have a great afternoon James.

2= Moderately empathic: Cases receiving a "2" start to show signs of empathy by asking questions or attempting to ease the mind of the patient. Additionally, within this category, participants are serving as a source of encouragement for the patient. Sample statements could include (but are not limited to) "I understand you aren't feeling well; I hope this medicine makes you feel better; etc."

Case 260: Hi AMy Jones, dr. Smith tells me you're not feeling well today. If I may ask, what is bothering you? Those symptoms sound a lot like the flu, which the doctor has recommended a medication for. The medicine you'll be taking is TAMIFLU and it is an antiviral, which means it will fight the virus that is ailing you. You'll only be on this for 5 days. SO it is not that bad. Hopefully this makes you feel somewhat better, since htis is not a major sickness. The doctor is making rounds and will be in shortly.

3= Most empathic: Cases receiving a " 3 " are demonstrated by participants understanding and acknowledging patient emotions while also offering emotional support. 
Additionally, within this category, participants reassure the patient that they will feel better.

Case: 261: Hi Amy, my name is Simon. I'm a nurse working with Dr. Smith today. How are you doing? / I'm sorry you're not feeling well. I know having the flu is the worst. Dr Smith let me know ther eis a medication we can offer you that can help you feel better faster. It's called Tamiflu - have you ever heard of it? / Right, it's a medication that fights the flu virus. It's works for stomache flu as well as other types of flu. You take it twice a day. One pill twice a day for five days. So that's one pill in the morning and one in the evening. Yes, take it for the full five days. You don't have to take it with food but my reccomendation is that you do. One of the side effects of the medication is nausea, but it's much better if you take the pill with food. I know, nausea is ecactly what you are tyring to avoid. But I think that if you are sure to take the pills with food you will have no nauseau or just ver minor nausea, and you should feel better faster than if you didn't take it. That's a good question. We can run that by Dr. Smith when he comes back in. Is there anything else I can help you with in the meantime? Can I get you a glass of water or a new magazine? Okay, I will go let Dr. Smith know you're ready to see him again. Thanks so much for coming to see us today. 


\title{
Appendix P: Sample Participant Simulation Response
}

\author{
Male Participant, Male Patient, Patient Management Condition
}

"Good morning, Mr. Jones. I show on your chart that your name is James Jones, with a birthdate of March 2, 1979, is this correct? Mr. Jones, or James, may I call you James? My name is Brian and I am Dr. Smith's personal nurse. Dr. Smith had to attend to an emergency but he wanted me to assure you that you are going to be fine. I am calling you in a prescription for Tamiflu to your listed pharmacy. You have contracted the flu and Dr. Smith said you need to stay home and in bed for 3 days. And, to be sure to come back to see him if things don't get better at the end of 3 days. You understand so far, James? Okay, your medicine will be in capsule form. It's best to eat a small snack at least before you take your medicine. You will one capsule, 3 times a day for 5 days. This Tamaflu will knock out this flu bug you have. Thank you James. Dr. Smith said to tell you he appreciates you visiting with him today and he is very sorry he had this emergency that required him to leave you in my hands. Thank you James. You come back or call us if you don't get better in 3 days. Take your records to the front for check out please and I hope you feel like yourself soon." Participant 156, Age: 21 


\title{
Appendix Q: Sample Participant Simulation Response
}

\author{
Male Participant, Male Patient, Empathy Condition
}

"Good afternoon Mr Jones, I understand that you aren't feeling so well huh? My name is John and I am a nurse here at Dr. Doe's office. James, if I may, it looks like you have caught the stomach bug, otherwise known as the stomach flu. I am sure you are feeling pretty miserable, but I can assure you that this is going to pass in the next day or two. The doctor has written a script for trimeflu, do you do well with swallowing pills? The dosage is one $75 \mathrm{mg}$ pill two times a day for the next five days. Typically, side effects are very rare, but may include increased stomach irritability and/or nausea. If you take the pills with some food, the side effects are greatly reduced. I have gone ahead and called your script into your pharmacy so you won't have to wait for it. At this time, do you have any questions James? Do you need a note for work or for your own records? If you think of anything at all after you leave, please do not hesitate to call. In the meantime, try to keep putting fluids in your system and get some rest. Thank you so much Mr Jones and I wish you a speedy recovery. Take care” Participant 87, Age: 25 


\title{
Appendix R: Sample Participant Simulation Response
}

\author{
Male Participant, Male Patient, Control Condition
}

"hI Ms. Jones, I'm Stephen and I'm a nurse here at the clinic. I'll be helping with the care for the stomach flu today. Please feel free to ask me any questoins, if I'm unable to answer them I'll relay them to the doctor when he returns to answer them for you. The doctor prescribed Tamiflu in capsue form. This will be taken twice per day for five days. You can take it on an empty stomach but some people experience stomach discomfort so you may take it with a light snack, milk or meal. Side effects you may experience are some nausea andvomiting. This might happen during the first two days or longer and may be reduced by taking with a light snack. Another possible side effect is an allergic reaction or rash, if either of these occur then stop taking Tamiflu and call our office right away. Again, you received a diagnosis of stomach flu which is a viral infection and Tamiflu will help to treat this. You'll take one capsule of tamiflu twice per day for five days. thank y ou for the chance to care for you today and please let me know if you have any questions." Participant 74, Age: 27 


\section{Appendix S: Sample Participant Simulation Response}

\section{Male Participant, Female Patient, Patient Management Condition}

"Hello Amy, I'm Nathan and I here you are feeling a under the weather. I am the R.N on staff and I am here to inform you of the medication you are receiving and the proper usage of the medication. Dr. Smith would normally handle this, but he personally asked me to take care of you while he was out, but he will be back to answer any questions before you leave today if you have any. The medication that you have been prescribed is called Tamiflu. Take one pill twice a day for 5 days. The most common side-effects of the medication are nausea and vomitting, which will most likely arise within 2 days.

However, it is reccomended to take them with a meal or a light snack to help prevent the side-effects from occuring, but you can take the pills without a meal or snack if you so choose. If you develop any sort of allergic reaction or severe rash while taking the pills contact us right away. Hope you start feeling better, if there is anything I can help you out with give the office a call otherwise Dr. Smith will be right back to answer any questions that you may have." Participant 141, Age: 18 


\title{
Appendix T: Sample Participant Simulation Response
}

\author{
Male Participant, Female Patient, Empathy Condition
}

"Hello Amy, how are you? My name is Christopher Henry, and I am the assistant to Dr. Smith, who has temporarily been called away for an emergency but should return shortly. While we await his return he has asked me to go over his diagnosis with you. Dr. Smith diagnosed you with influenza or stomach flu, which is quite common this time of year. He will prescribe you TAMIFLU. You should take 1 capsule of $75 \mathrm{mg}$ twice daily. You can take this with or without food, but taking TAMIFLU with a light snack, milk or meal seems to reduce the risk of side effects. You should take TAMIFLU for 5 days. There are some mild side effects, which are mainly nausea and vomiting, though not everyone will experience these side effects. If you are to have side effects, then you will most likely have those in the first 2 days. If you take TAMIFLU with a meal, then this may reduce the risk of side effects. If you experience allergic reactions or a rash, you should stop taking TAMIFLU immediately and contact us. Should you have any questions, Dr. Smith will return very soon and he can address any questions or concerns you may have. Thank you for being so patient and for your visit." Participant 182, Age: 23 


\section{Appendix U: Sample Participant Simulation Response}

\section{Male Participant, Female Patient, Control Condition}

"Hello Ms. Amy. My name is Cody and I've been a nurse here for 5 years. I'm sure here to te-just here to tell you what the doctor's diagnosis is and the medication he perscribed, as well as any thiing about the meds you should know. just until the the dr. returns. So the $d r$. is saying you may have influenza or stomach flu. and he wants to give you tamiflu. you take it orally every day. twice a day. its a $75 \mathrm{mg}$ pill. you may take it with food, but there is a chance you might get a stomach ache if you take with milk, or food. So you may see side effects from the meds, usually two days after you take them. usually just nausea or vomitting. if you get a rash, then stop taking and call the office right away. the symptoms or mild to moderate effects so if there is any issues dont hesitate to call. do you have any questions? Perfect, alright thanks you so much. i'll give you a written set of instructions later. let me know if you need any help or questions okay? okay thank you bye." Participant 354, Age: 19 


\section{Appendix V: Sample Participant Simulation Response}

\section{Female Participant, Male Patient, Patient Management Condition}

"Hello Mr. Jones. Dr. Smith wanted me to come in and inform you of your diagnosis and tell you about the medication he has prescribed. You have the stomach flue also knows as the influenza. This is a virus that cannot be treated with antibiotics, however, there is a medication called Tamiflu that may ease the symptoms. This medication can be taken with or without food, but it's bets to be taken with a light snack or milk. The order is prescribed for you to take 1 capsule by mouth twice daily for 5 days. You should be aware that the most common side effects of this medication are nausea and vomiting. However, if a rash develops or severe allergic rx sysmtoms occur, you should contact this office immediately. Now, I know you're not feeling well and that's what brought you in here. I can assure you that Dr. Smith will be in as promptly as possible to answer any questions you may have, however, he had an emergency to attend to and it may take a little bit of time. Do you have any questions that I can answer for you? Is there anything I can do for you while you wait?" Participant 258, Age: 19 


\title{
Appendix W: Sample Participant Simulation Response
}

\author{
Female Participant, Male Patient, Empathy Condition
}

"Good afternoon Mr. Jones, how are you feeling? Aching? How's your tummy? Yup, the flu is no fun at all. Dr. Smith is finishing up some paperwork, but he's asked me to come in and talk to you about your prescription, did he say anything about it? / Okay, so this is a prescription for Tamiflu which is going to help your body fight off the virus. You're going to take two capsules a day, one with breakfast and one with supper. If you don't feel up to a full breakfast see if you can keep down a piece of toast or some crackers or whatever your comfort food when you're sick. It might upset your stomach at first but that should fade by the second or third day. You're going to take it for five days, so if you start this evening your last dose is on [day] morning. Would you like me to write that down for you? / / Of course with any medication if you start getting a rash or swelling in your face or neck we want you to stop taking it and call our office or 911, whichever seems appropriate to the level of reaction. Do you have any questions?" Participant 126, Age: 19 


\title{
Appendix X: Sample Participant Simulation Response
}

\author{
Female Participant, Male Patient, Control Condition
}

"Hello Mr. Jones, I am the nurse Kim and Dr. Smith asked me to tell you he is sorry but he had an emergency and had to leave but he did want to make sure you are taken care of. Dr. Smith said you had a stomach virus and he is going to put you on a medicine called Tamiflu which you will take twice a day until it is all gone. You can taken it either with or without food but it is recommended that you take it with a light snack or a glass of milk in case it might give you a little stomach upset. As far as adverse reactions, few patients do develop some stomach upset when taking the medicine such as nausea, vomiting or diarrhea but Dr. Smith doesnt anticipate you having any such reaction. Also because it is a new medicine please be aware that an allergic reaction is possible. If you develop a rash, itching of your skin or anhy difficulty breathing then stop taking the medication immediately and contact our office for further instructions. Also if you have any questions in general please feel free to contact Dr. Smith and he can discuss any issues you may have. Is there anything that I need to explain to you again or do you have any questions? What pharmacy do you use so that Dr. Smith can call the medicine in for you. I do hope that you get to feeling better soon and, once again, Dr. Smith is very sorry that he had to leave but he did want to make sure you were taken care of. Thank you so much, Mr Jones, and do feel free to call if you need anything. ” Participant 16, Age: 26 


\section{Appendix Y: Sample Participant Simulation Response}

\section{Female Participant, Female Patient, Patient Management Condition}

"Hello Ms. Jones, my name is Laurissa and I am the nurse here in the office. The doctor asked me to come speak to you about the results of your exam today. Unfortunately, the doctor was called away to an emergency so he asked me to come in to answer any questions you may have. He wanted me to let you know that you have the stomach flu and that he is prescribing a medication for you called Tamiflu. Have you ever taken Tamiflu before? He has prescribed that you take 2 tablets each day for 5 days. The instructions will be included on the prescription bottle. Also, it is recommended that you do take the medication with a light snack to prevent getting the side effect of an upset stomach or throwing up. Do you have any questions about your condition or medication that I can answer for you? Thank you for coming in today. I apologize that the doctor had to leave so suddenly and we really appreciate your patience. I hope you feel better soon. Please call us if you have any further questions." Participant 56, Age: 24 


\section{Appendix Z: Sample Participant Simulation Response}

\section{Female Participant, Female Patient, Empathy Condition}

"Hi there, my name is Nicole and I am a nurse here in Dr. Jones' ofice. There has been an emergency the Dr. has had to attend to so he has asked me to come talk to you so we dont have to keep you waiting here! Dr. Jones has diagnosed you with influenza, or the common stomach flu. He has prescribed you a medication called Tamiflue. You are going to take this 2 times a day for 5 days. This does sometimes cause more nausea and vomitting, so it's best that you take it with a small meal, or a snack, or even a glass of milk. You dont have to, but we do recommend it so that you dont get an upset stomach, well even more of one! Now with this medication there is a risk of having an allergic reaction. If you begin to show signs of that we want you to call right away or come to the emergency room. Signs of an allergic reaction are a rash, swelling of the tongue or mouth, hives, or anything like that. Do you have any questions? Ok, well if you think of any the doctor is going to pop in here real quick before you leave, give me just a minute and I will go let him know you are all set. Thanks for your understanding, have a wonderful day!" Participant 211, Age: 20 


\title{
Appendix AA: Sample Participant Simulation Response
}

\author{
Female Participant, Female Patient, Control Condition
}

"Hello Mrs. Jones. I am your nurse, Diane Sparks and I will be explaining your diagnosis and medication instructions today. After I finish, it'll be just a few minutes and Dr. Smith will return. You have been diagnosed with Influenza or stomach flu. Dr. Smith has prescribed you a medication called TAMIFLU. You are to take one capsule orally, twice a day for five days. This medication can be taken with or without food. Sometimes, taking TAMIFLU on an empty stomach can cause stomach upset, nausea or vomiting. If you experience these symptoms, you should TAMIFLU after a light snack or meal. You can even have a glass of milk before taking it to avoid any further stomach upset. Vomiting and nausea are the most common side effects that occur while taking TAMIFLU. These side effects will generally be mild or moderate and the symptoms will appear after a couple days of taking the medication. Eating food with TAMIFLU may help to decrease the risk of side effects. If any of these side effects become bothersome, you develop a rash or severe allergic reaction occurs, stop taking TAMIFLU right away and call our front office. Do you have any questions? Thanks you for your patience. Dr. Smith will be in to see you momentarily." Participant 300, Age: 19 


\section{References}

Abu-Rabia, S. (2004). Teacher's role, learner's gender differences, and FL anxiety among seven-grade students studying English as a FL. Educational Psychology, 24, 711-721.

Aiken, L. H., Clarke, S. P., Sloane, D. M., Sochalski, J. A., \& Silber, J. H. (2002). Hospital nurse staffing and patient mortality, nurse burnout, and job dissatisfaction. The Journal of the American Medical Association, 288, 1987-1993.

Ali, J., Cohen, R. J., Gana, T. J., \& Al-Bedah, K. F. (1998). Effect of the advanced trauma life support program on medical students' performance in simulated trauma patient management. Journal of Trauma, Injury Infection, \& Critical Care, 44, 588-591.

Allport, G. W. (1954). The nature of prejudice. Reading, MA: Perseus Books.

Alpert, D., \& Breen, D. T. (1989). Liberality in children and adolescents. Journal of Vocational Behavior, 34, 154-160.

American Association of Colleges of Nursing. (2006). Your nursing career: A look at the facts. Retrieved from http://www.aacn.nche.edu/Education/nurse_ed/career.htm

Anker, R. (1998). Gender and jobs: Sex segregation of occupations in the world. Geneva, Switzerland: International Labour Office.

Anthony, A. S. (2004). Gender bias and discrimination in nursing education: Can we change it? Nurse Educator, 29, 121-125.

Anthony, A. S. (2006). Tear down the barriers of gender bias. Men in Nursing, 1, 43-49. 
Apker, J. Zabava Ford, W. S., \& Fox, D. H. (2003). Predicting nurses' organizational and professional identification: The effect of nursing roles, professional autonomy, and supportive communication. Nursing Economics, 21, 226-232.

Arborelius, E., \& Bremberg, S. (1992). What can doctors do to achieve a successful consultation? Videotaped interviews analyzed by "consultation map" method. Family Practice, 9, 61-66.

Aries, E. (1996). Men and women in interaction: Reconsidering the differences. New York: Oxford University Press.

Aronson, J. (2002). Stereotype threat: Contending and coping with unnerving expectations. In J. Aronson (Ed.), Improving academic achievement: Impact of psychological factors on education (pp. 279-304). San Diego, CA: Academic Press.

Aronson, J., Lustina, M., Good, C., Keough, K. Steele, C., \& Brown, J. (1999). When white men can't do math: Necessary and sufficient factors in stereotype threat. Journal of Experimental Social Psychology, 35, 29-46.

Aronson, J., \& McGlone, M. S. (2009). Social identity and stereotype threat. In T. Nelson (Ed.), Handbook of stereotyping, prejudice, and discrimination research (pp. 153178). New York: Psychology Press.

Auerbach, D. I., Buerhaus, P. I., \& Staiger, D. O. (2011). Registered nurse supply grows faster than projected amid surge in new entrants ages 23-26. Health Affairs, 30, 2286-2292. 
Azoulay, E., Chevret, S., Leleu, G., Pochard, F., Barboteu, M., Adrie, C., ..., Schlemmer, B. (2000). Half the families of intensive care unit patients experience inadequate communication with physicians. Critical Care Medicine, 28, 3044-3049.

Bakan, D. (1966). The duality of human existence: An essay on psychology and religion. Skokie, IL: Rand McNally.

Bales, R. (1950). Interaction process analysis. Reading, MA: Addison-Wesley.

Baron, R. M., \& Kenny, D. A. (1986). The moderator-mediator variable distinction in social psychological research: Conceptual, strategic, and statistical considerations. Journal of Personality and Social Psychology, 51, 1173-1182.

Barrere, C. C. (2007). Discourse analysis of nurse-patient communication in a hospital setting: Implications for staff development. Journal for Nurses in Staff Development, 23, 114-122.

Barrows, H. (1993). An overview of the uses of standardized patients for teaching and evaluating clinical skills. Academic Medicine, 68, 443-453.

Baumeister, R. F. (1984). Choking under pressure: Self-consciousness and paradoxical effects of incentives on skillful performance. Journal of Personality and Social Psychology, 46, 610-620.

Beck, R. S., Daughtridge, R., \& Sloane, P. D. (2002). Physician-patient communication in the primary care office: A systematic review. Journal of the American Board of Family Medicine, 15, 25-38.

Beeman, W. O. (2001). Writing for the crisis. Anthropology Today, 17(6), 1-2. 
Beggs, J. M., \& Doolittle, D. C. (1993). Perceptions now and then of occupational sex typing: A replication of Shinar's 1975 study. Journal of Applied Social Psychology, 23, 1435-1453.

Bell-Scriber, M. (2008). Warming the nursing education climate for traditional-age learners who are male. Nursing Education Research, 29, 143-150.

Bem, S. L. (1974). The measurement of psychological androgyny. Journal of Consulting and Clinical Psychology, 42, 155-162.

Ben-Zeev, T., Fein, S., \& Inzlicht, M. (2005). Arousal and stereotype threat. Journal of Experimental Social Psychology, 41, 174-181.

Benbow, C. P., \& Stanley, J. C. (1980). Sex differences in mathematical ability: Fact or artifact? Science, 210, 1262-1264.

Bergen, D. J., \& Williams, J. E. (1991). Sex stereotypes in the United States revisited: 1972-1988. Sex Roles, 24, 413-423.

Berger, C. R., \& Jordan, J. M. (1992). Planning sources, planning difficulty and verbal fluency. Communication Monographs, 59, 130-149.

Bernard Hodes Group. (2005). Men in nursing survey. Retrieved from www.hodes.com/ industries/healthcare/resources/research/meninnursingsurvey.asp

Berryman, C. L., \& Wilcox, J. R. (1980). Attitudes toward male and female speech: Experiments on the effects of sex-typed language. Western Journal of Speech Communication, 44, 50-59.

Berwick, D. M., \& Leape, L. L. (1999). Reducing errors in medicine. British Medical Journal, 319, 136-137. 
Betz, N. E., \& Hackett, G. (1983). The relationship of mathematics self-efficacy expectations to the selection of science-based college majors. Journal of Vocational Behavior, 23, 329-345.

Blackwell, B. (1996). From compliance to alliance. A quarter century of research. Netherlands Journal of Medicine, 48, 140-149.

Blascovich, J., Spencer, S. J., Quinn, D., \& Steele, C. M. (2001). African Americans and high blood pressure: The role of stereotype threat. Psychological Science, 12, 225-229.

Boehm, F. H. (2008). Teaching and learning moments: Teaching bedside manners to medical students. Academic Medicine, 83, 534.

Booth-Butterfiled, M., \& Butterfield, S. (1986). Effects of evaluation, task structure, trait$\mathrm{CA}$, and reticence on state-CA and behavioral disruption in dyadic settings. Communication Monographs, 53, 144-159.

Bosson, J. K., Brown, R. P., Zeigler-Hill, \& Swann, W. B. (2003). Self-enhancement tendencies among people with high explicit self-esteem: The moderating role of implicit self-esteem. Self and Identity, 2, 169-187.

Boughn, S. (1994). Why do men choose nursing? Nursing and Health Care, 25, 406-411.

Bradac, J. J., Mulac, A., \& Thompson, S. A. (1995). Men's and women's use of intensifiers and hedges in problem-solving interaction: Molar and molecular analyses. Research on Language and Social Interaction, 29, 93-116.

Bradley, H. (1993). Across the great divide: The entry of men into women's jobs. In C. Williams (Ed.), Doing women's work: Men in non-traditional occupations (pp. 10-27). Newbury Park, CA: Sage. 
Brady, M. S., \& Sherrod, D. R. (2003). Retaining men in nursing programs designed for women. Journal of Nursing Education, 42, 159-162.

Brannon, R. (1976). The male sex role: Our culture's blueprint for manhood, what it's done for us lately. In D. David \& R. Brannon (Eds.), The forty-nine percent majority: The male sex role. Reading, MA: Addison-Wesley.

Breckinridge, B., Tollison, A. C., \& McGlone, M. S. (2011, November). Are white people racist?: Stereotype threat in interracial interaction. Paper presented at the $97^{\text {th }}$ annual convention of the National Communication Association, New Orleans, LA.

Bresnahan, M. J., Inoue, Y., Liu, W. Y., \& Nishida, T. (2001). Changing gender roles in prime-time commercials in Malaysia, Japan, Taiwan, and the Unites States. Sex Roles, 45, 117-131.

Brilowski, G. A., \& Wendler, M. C. (2005). An evolutionary concept analysis of caring. Journal of Advanced Nursing, 50, 641-650.

Briton, N. J., \& Hall, J. A. (1995). Beliefs about female and male nonverbal communication. Sex Roles, 32, 79-90.

Broverman, I., Vogel, S., Broverman, D., Clarkson, F., \& Rosenkrantz, P. (1972). Sexrole stereotypes: A reappraisal. Journal of Social Issues, 28, 59-78.

Brown, R. P., \& Josephs, R. A. (1999). A burden of proof: Stereotype relevance and gender differences in math performance. Journal of Personality and Social Psychology, 76, 246-257.

Bullough, V. L. (1994). History: Men, women, and nursing history. Journal of Professional Nursing, 10, 127. 
Bureau of Labor Statistics. (2012). Occupational outlook handbook: Registered nurses. Retrieved from http://www.bls.gov/ooh/Healthcare/Registered-nurses.htm Burt, K. (1998). Male nurses still face bias. American Journal of Nursing, 98, 64-65. Cameron, C., \& Moss, P. (1998). Men as carers for children: An introduction. In C. Owen, C. Cameron, \& P. Moss (Eds.), Men as workers in services for young children: Issues of a mixed gender workforce. London: Institute of Education.

Campbell, A. (1984). Moderated love: A theology of professional care. London: SPCK.

Campbell, T. A., Auerbach, S. M., \& Kiesler, D. J. (2007). Relationship of interpersonal behaviors and health-related control appraisals to patient satisfaction and compliance in a university health center. Journal of American College Health, 55, $333-340$.

Campbell, T., Gillaspy, J. A., Jr., \& Thompson, B. (1997). The factor structure of the Bem Sex-Role Inventory (BSRI): Confirmatory analysis of long and short forms. Educational and Psychological Measurement, 57, 118-124.

Cappella, J. (1985). Controlling the floor in conversation. In A. Siegman \& S. Feldstein (Eds.), Multichannel integrations of nonverbal behavior (pp. 69-103). Hillsdale, NJ: Erlbaum.

Carli, L. L. (1990). Gender, language, and influence. Journal of Personality and Social Psychology, 59, 941-951.

Chaplin, T. M., Cole, P. M., \& Zahn-Waxler, C. (2005). Parental socialization of emotion expression: Gender differences and relations to child adjustment. Emotion, 5, 8088.

Christman, L. (1988). Men in nursing. Annual Review of Nursing Research, 6, 193-205. 
Chur-Hansen, A. (2002). Preferences for female and male nurses: The role of age, gender and previous experience - year 2000 compared with 1984. Issues and Innovations in Nursing Practice, 37, 192-198.

Chusmir, L. H. (1990). Men who make nontraditional career choices. Journal of Counseling and Development, 69, 11-16.

Cockburn, C. (1988). The gendering of jobs: Workplace relations and the reproduction of sex segregation. In S. Walby (Ed.), Gender segregation at work. Philadelphia: Open University Press.

Comer, S. K. (2005). Patient care simulations: Role playing to enhance clinical understanding. Nursing Education Perspectives, 26, 357-361.

Connell, R. W. (1995). Masculinities. Oxford: Polity Press.

Connell, R. W. (2000). The men and the boys. Cambridge: Polity Press.

Connolly, C., \& Rogers, N. (2005). Who is the nurse?: Rethinking the history of gender and medicine. OAH Magazine of History, 19, 45-49.

Cortes, D. E., Mulvaney-Day, N., Fortuna, L., Reinfeld, S., \& Alegría, M. (2009). Patient-provider communication: Understanding the role of patient activation for Latinos in mental health treatment. Health Education \& Behavior, 36, 138-154.

Costanzo, M., \& Archer, D. (1993a). The Interpersonal Perception Task-15 (IPT-15). Berkeley, CA: University of California Center for Media and Independent Learning.

Costanzo, M., \& Archer, D. (1993b). The Interpersonal Perception Task-15 (IPT-15): A guide for researchers and teachers. Berkeley, CA: University of California Center for Media and Independent Learning. 
Cottrell, N. B. (1972). Social facilitation. In C. G. McClintock (Ed.), Experimental social psychology (pp. 185-236). New York: Holt, Rinehart \& Winston.

Coulehan, J. L., Platt, F. W., Egener, B., Frankel, R., Lin, C., Lown, B., \& Salazar, W. H. (2001). "Let me see if I have this right...": Words that build empathy. Annals of Internal Medicine, 135, 221-227.

Couper, M. P. (2005). Technology trends in survey data collection. Social Science Computer Review, 23, 486-501.

Coutu, D. L. (2002). How resilience works. Harvard Business Review, 80, 46-50.

Cozby, C. (1973). Self-disclosure: A literature review. Psychological Bulletin, 79, 73-91.

Crocker, J., Major, B., \& Steele, C. (1998). Social stigma. In D. T. Gilbert, S. T. Fiske, \& G. Lindzey (Eds.), Handbook of social psychology (4 ${ }^{\text {th }}$ ed., pp. 504-553). Boston: McGraw-Hill.

Croizet, J., \& Claire, T. (1998). Extending the concept of stereotype threat to social class: The intellectual underperformance of students from low socioeconomic backgrounds. Personality and Social Psychology, 24, 588-595.

Cudé, G., \& Winfrey, K. (2007). The hidden barrier. Gender bias: Fact or Fiction? Nursing for Women's Health, 11, 255-265.

D’Antonio, P., \& Whelan, J. C. (2009). Counting nurses: The power of historical census data. Journal of Clinical Nursing, 18, 2717-2724.

Daly, J. A., \& Buss, A. (1984). The transitory causes of audience anxiety. In J. A. Daly \& J. C. McCroskey (Eds.), Avoiding communication: Communication apprehension, reticence, and shyness (pp. 67-78). Beverly Hills, CA: Sage. 
Daly, J. A., \& Miller, M. D. (1975). The empirical development of an instrument to measure writing apprehension. Research in the Teaching of English, 9, 242-249.

Dassen, T. W., Nijhuis, F. J., \& Philipsen, H. (1990). Male and female nurses in intensive-care wards in the Netherlands. Journal of Advanced Nursing, 15, 387393.

Davies, C. (1995). Gender and the professional predicament in nursing. Buckingham: Open University Press.

Davies, P. G., Spencer, S. J., \& Steele, C. M. (2005). Clearing the air: Identity safety moderates the effects of stereotype threat on women's leadership aspirations. Journal of Personality and Social Psychology, 88, 276-287.

Davis, D. W., \& Silver, B. D. (2003). Stereotype threat and race of interviewer effects in a survey on political knowledge. American Journal of Political Science, 47, 33-45.

Davis, M. T., \& Bartfay, W. J. (2001). Men in nursing: An untapped resource. Canadian Nurse, 97, 14-18.

Deaux, K. (1995). How basic can you be? The evolution of research on gender stereotypes. Journal of Social Issues, 51, 11-20.

Dennis, M. R., \& Kunkel, A. D. (2004). Perceptions of men, women and CEOs: The effects of gender identity. Social Behavior and Personality, 32, 155-172.

Devine, P. G., \& Elliot, A. J. (1995). Are racial stereotypes really fading? The Princeton trilogy revisited. Personality and Social Psychology Bulletin, 21, 1139-1150.

DiPalo, M. T. (1997). Rating satisfaction research: Is it poor, fair, good, very good or excellent? Arthritis Care and Research, 10, 422-430. 
Dixon, T. L. (2006). Psychological reactions to crime news portrayals of black criminals: Understanding the moderating roles of prior news viewing and stereotype endorsement. Communication Monographs, 73, 162-187.

Domke, D., McCoy, K., \& Torres, M. (1999). News media, racial perceptions, and political cognition. Communication Research, 26, 570-607.

Donahue, M. P. (1996). Nursing. The finest art: An illustrated history $\left(2^{\text {nd }}\right.$ ed.). St. Louis: Mosby.

Donaldson, M. (1993). What is hegemonic masculinity? Theory and Society, 22, 643-657.

Dovidio, J. F., Brown, C., Heltman, K., Ellyson, S., \& Keating, C. (1988). Power displays between women and men in discussions of gender-linked tasks: A multi-channel study. Journal of Personality and Social Psychology, 55, 580-587.

Dovidio, J. F., \& Gaertner, S. L. (1986). Prejudice, discrimination and racism: Historical trends and contemporary approaches. In Prejudice, discrimination and racism (pp. 1-30). Orlando, FL: Academic Press.

Dreger, R. M., \& Miller, S. K. (1960). Comparative psychological studies of Negroes and Whites in the United States. Psychological Bulletin, 57, 361-402.

Dubrovsky, V. J., Kiesler, S., \& Sethna, N. (1991). The equalization phenomenon: Status effects in computer-mediated and face-to-face decision-making groups. HumanComputer Interaction, 6, 119-146.

Duehr, E. E., \& Bono, J. E. (2006). Men, women, and managers: Are stereotypes finally changing? Personnel Psychology, 59, 815-846.

Eagly, A. H. (1987). Sex differences in social behavior: A social-role interpretation. Hillsdale, NJ: Erlbaum. 
Eagly, A. H., \& Steffen, V. J. (1984). Gender stereotypes stem from the distribution of women and men into social roles. Journal of Personality and Social Psychology, $46,735-754$.

Eccles, J. S. (1987). Gender roles and women's achievement-related decisions. Psychology of Women Quarterly, 11, 135-172.

Egeland, J. W., \& Brown, J. S. (1988). Sex role stereotyping and role strain of male registered nurses. Research in Nursing \& Health, 11, 257-267.

Egbert, N., \& Parrott, R. (2003). Empathy and social support for the terminally ill: Implicatoins for recruiting and retaining hospice and hospital volunteers. Communication Studies, 54, 18-34.

Ekstrom, D. N. (1999). Gender and perceived nurse caring in nurse-patient dyads. Journal of Advanced Nursing, 29, 393-401.

Entman, R. M., \& Rojecki, A. (2000). The Black image in the White mind: Media and race in America. Chicago: University of Chicago Press.

Evans, J. A. (2002). Cautious caregivers: Gender stereotypes and the sexualization of men nurses' touch. Journal of Advanced Nursing, 40, 441-448.

Evans, J. A. (1997). Men in nursing: Issues of gender segregation and hidden advantage. Journal of Advanced Nursing, 26, 226-231.

Fealy, G. M. (1995). Professional caring: The moral dimension. Journal of Advanced Nursing, 22, 1135-1140.

Fecteau, T. J., Jackson, J., \& Dindia, K. (1992). Gender orientation scales: An empirical assessment of content validity. In L. Perry, L. Turner, \& H. M. Sterk (Eds.), Constructing and reconstructing gender: The links among communication, 
language, and gender (pp. 17-34). Albany, NY: State University of New York Press.

Feinstein, A. R. (1997). System, supervision, standards, and the 'epidemic' of negligent medical errors. Archives of Internal Medicine, 157, 1285-1289.

Finch, L. P. (2005). Nurses' communication with patients: Examining relational communication dimensions and relationship satisfaction. International Journal for Human Caring, 9, 14-23.

Finfgeld-Connett, D. (2008). Qualitative convergence of three nursing concepts: Art of nursing, presence and caring. Journal of Advanced Nursing, 63, 527-534.

Finkel, S. E., Guterbock, T. M., \& Borg, M. J. (1999). Race-of-interviewer effects in a preelection poll: Virginia 1989. Public Opinion Quarterly, 55, 313-330.

Finlayson, B., Dixon, J., Meadows, S., \& Blair, G. (2002). Mind the gap: The extent of the NHS nursing shortage. British Medical Journal, 325, 538-541.

Flannery, D. D. (2000). Identity and self-esteem. In E. Hayes \& D. D. Flannery (Eds.), Women as learners: The significance of gender in adult learning (pp. 53-78), San Francisco, CA: Jossey-Bass.

Fleischer, S., Berg, A., Zimmermann, M., Wuste, K., \& Behrens, J. (2009). Nurse-patient interaction and communication: A systematic literature review. Journal of Public Health, 17, 339-353.

Fouad, N. A., \& Smith, P. L. (1996). A test of a social cognitive model for middle school students: Math and science. Journal of Counseling Psychology, 43, 338-346.

Fox, J. G., \& Storms, D. M. (1981). A different approach to sociodemographic predictors of satisfaction with health care. Social Science and Medicine, 15A, 557-564. 
Ford, S., Fallowfield, L., \& Lewis, S. (1996). Doctor-patient interactions in oncology. Social Science \& Medicine, 42, 1511-1519.

Gaberson, K. B., \& Oermann, M. H. (1999). Clinical teaching strategies in nursing. New York: Springer Publishing.

Gainor, K. A., \& Lent, R. W. (1998). Social cognitive expectations and racial identity attitudes in predicting the math choice intentions of Black college students. Journal of Counseling Psychology, 45, 403-413.

Galbraith, M. (1992). Understanding career choices of men in elementary education. The Journal of Education Research, 85, 246-253.

Gallie, K. (2005). Student attrition before and after modifications in distance course delivery. Studies in Learning, Evaluation Innovation and Development, 2, 69-76.

Gardner, J. (2005). A successful minority retention project. Journal of Nursing Education, 44, 566-569.

Garrity, T. F. (1981). Medical compliance and clinician-patient relationship: A review. Social Science Medicine, 25, 215-222.

Geary, D. C. (1996). Sexual selection and sex differences in mathematical abilities. Behavioral and Brain Sciences, 19, 229-284.

Geissler, E. M. (1986). Nurturance: Old friend, new word. Journal of Nursing History, 2, 61-65.

Gilchrist, K. L., \& Rector, C. (2007). Can you keep them? Strategies to attract and retain nursing students from diverse populations: Best practices in nursing education. Journal of Transcultural Nursing, 18, 277-285. 
Gilbert, G. M. (1951). Stereotype persistence and change among college students. Journal of Personality and Social Psychology, 46, 245-254.

Giles, H., Scholes, J., \& Young, L. (1983). Stereotypes of male and female speech: A British study. Central States Speech Journal, 34, 255-256.

Gilliam, F., \& Iyengar, S. (2000). Prime suspects: The influence of local television news on the viewing public. American Journal of Political Science, 44, 560-573.

Godfrey, K. (2000). Don't believe the hype. Nursing Times, 96, 28-29.

Godkin, J., \& Godkin, L. (2004). Caring behaviors among nurses: Fostering a conversation of gestures. Health Care Management Review, 29, 258-267.

Goffman, E. (1963). Stigma. Englewood Cliffs, NJ: Prentice-Hall.

Goffman, E. (1973). Stigma: Notes on the management of spoiled identity. Harmondsworth: Penguin.

Golin, C. E., DiMatteo, M. R., \& Gelberg, L. (1996). The role of patient participation in the doctor visit. Diabetes Care, 19, 1153-1164.

Gonzales, P., Blanton, H., \& Williams, K. (2002). The effects of stereotype threat and double-minority status on the test performance of Latino women. Personality and Social Psychology Bulletin, 28, 659-670.

Goodnow, M. (1940). Outlines of nursing history $\left(6^{\text {th }}\right.$ ed. $)$. Philadelphia: W.B. Saunders Company.

Graddy, D. B. (2006). Gender salience and the use of linguistic qualifiers and intensifiers in online course discussions. American Journal of Distance Education, 20, 211229. 
Greene, J., \& Sparks, G. (1983). Explication of a cognitive model of communication apprehension: A new look at an old construct. Human Communication Research, 9, 349-366.

Greenfield, S., Kaplan, S. H., \& Ware, J. E., Jr. (1985). Expanding patient involvement in care: Effects on patient outcomes. Annals of Internal Medicine, 102, 520-528.

Greenfield, S., Kaplan, S. H., Ware, J. E., Jr., Yano, E. M., \& Frank, H. J. (1988). Patients' participation in medical care: Effects on blood sugar control and quality of life in diabetes. Journal of General Internal Medicine, 3, 448-457.

Gregersen, T. (2003). To err is human: A reminder to teachers of language-anxious students. Foreign Language Annals, 36, 25-32.

Grob, L. M., Meyers, R. A., \& Schuh, R. (1997). Powerful/powerless language use in group interactions: Sex differences or similarities. Communication Quarterly, 45, 282-303.

Guéguen, N., Lourel, M., Charron, C., Fischer-Lokou, \& Lamy, L. (2009). A web replication of Snyder, Decker, and Bersheid's (1977) experiment on the selffulfilling nature of social stereotypes. The Journal of Social Psychology, 149, $600-602$.

Hagerty, B. M., \& Patusky, K. L. (2003). Reconceptualising the nurse-patient relationship. Image: Journal of Nursing Scholarship, 35, 145-150.

Hajek, C., Abrams, J. R., \& Murachver, T. (2004). Female, straight, male, gay, and worlds betwixt and between: An intergroup approach to sexual gender identities. In J. Harwood \& H. Giles (Eds.), Intergroup communication: Multiple perspectives (pp. 43-64). New York: Peter Lang. 
Halim, M. L., Ruble, D. N., \& Amodio, D. M. (2011). From pink frilly dresses to 'one of the boys': A social-cognitive analysis of gender identity development and gender bias. Social and Personality Psychology Compass, 5, 933-949.

Hannah, A., \& Murachver, T. (1999). Gender and conversational style as predictors of conversational behavior. Journal of Language and Social Psychology, 18, 153174.

Harding, T. (2007). The construction of men who are nurses as gay. Journal of Advanced Nursing, 60, 636-644.

Hart, K. A. (2005). What do men in nursing really think?: Survey respondents speak out. Nursing, 35, 46-48.

Hartrick, G. (1997). Relationship capacity: The foundation for interpersonal nursing practice. Journal of Advanced Nursing, 26, 523-528.

Heilman, M. (2001). Description and prescription: How gender stereotypes prevent women's ascent up the organizational ladder. Journal of Social Issues, 57, 657674.

Hekman, D. R., Steensma, H. K., Bigley, G. A., \& Hereford, J. F. (2009). Effects of organizational and professional identification on the relationship between administrators' social influence and professional employees' adoption of new work behavior. Journal of Applied Psychology, 94, 1325-1335.

Heller, K. A., \& Ziegler, A. (1996). Gender differences in mathematics and the sciences: Can attributional retraining improve the performance of gifted females? Gifted Child Quarterly, 40, 200-210. 
Henbest, R. J., \& Fehrsen, G. S. (1992). Patient-centredness: Is it applicable outside the West? It's measurement and effect on outcomes. Family Practice, 9, 311-317.

Henson R.H. (1997). Analysis of the concept of mutuality. Image: Journal of Nursing Scholarship 29, 77-81.

Hernstein, R. J., \& Murray, C. (1994). The bell curve, intelligence and class structure in American life. New York: Free Press.

Hess, T. M., \& Hinson, J. T. (2006). Age-related variation in the influences of aging stereotypes on memory in adulthood. Psychology and Aging, 21, 621-625.

Hickson, G. B., Clayton, E. W., Githens, P. B., \& Sloan, F. A. (1992). Factors that prompted families to file medical malpractice claims following perinatal injuries. Journal of the American Medical Association, 267, 1359-1363.

Hilpert, F., Kramer, C., \& Clark, R. A. (1975). Participants' perceptions of self and partner in mixed sex dyads. Central States Speech Journal, 26, 52-56.

Holland, J. L. (1973). Making vocational choices: A theory of careers. Englewood Cliffs, NJ: Prentice-Hall.

Honeycutt, J. M. (2010). Introduction. In J. M. Honeycutt (Ed.), Imagine that: Studies in imagined interactions (pp. 1-14). Cresskill, NJ: Hampton Press.

Honeycutt, J. M. (2008). Imagined interaction theory: Mental representations of interpersonal communication. In L. Baxter and D. Braithwaite (Eds.), Engaging theories in interpersonal communication: Multiple perspectives (pp. 77-87). Thousand Oaks, CA: Sage.

Honeycutt, J. M. (2003). Imagined interactions: Daydreaming about communication. Cresskill, NJ: Hampton Press. 
Horrocks, S., Anderson, E., \& Salisbury, C. (2002). Systematic review of whether nurse practitioners working in primary care can provide equivalent care to doctors. British Medical Journal, 324, 819-823.

Horwitz, E. K. (2001). Language anxiety and achievement. Annual Review of Applied Linguistics, 21, 112-126.

Hubbard, A. E. (2001). Conflict between relationally uncertain romantic partners: The influence of relational responsiveness and empathy. Communication Monographs, $68,400-414$

Hummert, M. L., Shaner, J. L., Garstka, T. A., \& Henry, C. (1998). Communication with older adults: The influence of age stereotypes, context, and communicator age. Human Communication Research, 25, 124-151.

Ingersoll, G. L., Kirsch, J. C., Merk, S. E., \& Lightfoot, J. (2000). Relationship organizational culture and readiness for change to employee commitment to the organization. Journal of Nursing Administration, 30, 11-20.

Inoue, M., Chapman, R., \& Wynaden, D. (2006). Male nurses' experiences of providing intimate care for women clients. Journal of Advanced Nursing, 55, 559-567.

Inzlicht, M., \& Ben-Zeev, T. (2000). A threatening intellectual environment: Why females are susceptible to experiencing problem-solving deficits in the presence of males. Psychological Science, 11, 365-371.

Jacobs, J. E. (1993). Men in female-dominated fields. Trends and turnover. In C. Williams (Ed.), Doing “women's work”: Men in nontraditional occupations (pp. 49-63). London: Sage. 
Jacobs, J. E., \& Eccles, J. S. (1992). The impact of mothers' gender-role stereotypic beliefs on mothers' and children's ability perceptions. Journal of Personality and Social Psychology, 63, 932-944.

Ji, P. Lapan, R., \& Tate, K. (2004). Vocational interests and career efficacy expectations $\mathrm{n}$ relation to occupational sex-typing beliefs in eighth grade students. Journal of Career Development, 31, 143-154.

Joint Commission on Accreditation of Healthcare Organizations. (2007). Health care at the crossroads: Strategies for addressing the evolving nursing crisis. Retrieved from www.jointcommission.org/assets/1/.../health_care_at_the_crossroads.pdf

Jome, L. M., \& Toker, D. M. (1998). Dimensions of masculinity and major choice traditionality. Journal of Vocational Behavior, 52, 120-134.

Jones, J. M. (2011, December 12). Record 64\% rate honesty, ethics of members of Congress low: Ratings of nurses, pharmacists, and medical doctors most positive. Gallup Politics. Retrieved from http://www.gallup.com/poll/ 151460/record-ratehonesty-ethics-members-congress-low.aspx

Kalisch, P. A., \& Kalisch, B. J. (1978). The advance of American nursing. Boston: Little, Brown and Company.

Kaplan, S. H., Greenfield, S., \& Ware, J. E., Jr. (1989). Assessing the effects of physician-patient interactions on the outcomes of chronic disease. Med Care, 27, S110-127.

Karlins, M., Coffman, T. L., \& Walters, G. (1969). On the fading of social stereotypes: Studies in three generations of college students. Journal of Personality and Social Psychology, 13, 1-16. 
Katz, D., \& Braly, K. (1933). Racial stereotypes in one hundred college students. Journal of Abnormal and Social Psychology, 28, 280-290.

Katz, I., \& Benjamin, L. (1960). Effects of white authoritarianism in biracial work group. Journal of Abnormal Social Psychology, 61, 448-456.

Katz, I., \& Cohen, M. (1962). The effects of training Negroes upon cooperative problem solving in biracial teams. Journal of Abnormal and Social Psychology, 64, 319325.

Katz, I., Epps, E. G., \& Axelson, L. J. (1964). Effect upon Negro digit symbol performance of comparison with Whites and with other Negroes. Journal of Abnormal and Social Psychology, 69, 963-970.

Katz, I., Goldstone, J., \& Benjamin, L. (1958). Behavior and productivity in biracial work groups. Human Relations, 11, 32-38.

Katz, I., \& Greenbaum, C. (1963). Effects of anxiety, threat, and racial environment on task performance of Negro college students. Journal of Abnormal and Social Psychology, 66, 562-567.

Katz, I., Roberts, S. O., \& Robinson, J. M. (1965). Effects of task difficulty, race of administrator, and instructions on digit-symbol performance of Negroes. Journal of Personality and Social Psychology, 2, 53-59.

Kauppinen-Toropainen, K., \& Lammi, L. (1993). Men in female-dominated occupations: A cross-cultural comparison. In C. L. Williams (Eds.), Doing “women's work”: Men in nontraditional occupations (pp. 91-112). Newbury Park, CA: Sage.

Kelly, N. R., Shoemaker, M., \& Steele, T. (1996). The experience of being a male student nurse. Journal of Nursing Education, 35, 170-174. 
Kenny, D. A., Kashy, D. A., \& Bolger, N. (1998). Data analysis in social psychology. In D. Gilbert, S. T. Fiske, \& G. Lindzey (Eds.), Handbook of social psychology $\left(4^{\text {th }}\right.$ ed, Vol. 1, pp. 233-265). New York: McGraw-Hill.

Keogh, B., \& O'Lynn, C. (2007). Male nurses' experiences of gender barriers: Irish and American perspectives. Nurse Educator, 32, 256-259.

Keppel, G., Saufley, W. H., \& Tokunaga, H. (1992). Introduction to design and analysis: A student's handbook ( $2^{\text {nd }}$ ed). New York: W. H. Freeman and Company.

Kerssens, J. J., Bensing, J. M., \& Andela, M. G. (1997). Patient preference for genders of health professionals. Social Science and Medicine, 44, 1531-1540.

Kimmel, M. S. (1997). Masculinity as homophobia: Fear, shame, and silence in the construction of gender. In M. M. Gergen \& S. N. Davis (Eds.), Toward a new psychology of gender. A reader (pp. 223-224). New York: Routledge.

Kimura, D. (1992). Sex differences in the brain. Scientific American, 267, 81-87.

Kinnersley, P., Stott, N., Peters, T. J., \& Harvey, I. (1999). The patient-centeredness of consultations and outcomes in primary care. British Journal of General Practice, $49,711-716$.

Kippenbrock, T. (1990). School of nursing variables related to male student college choice. Journal of Nursing Education, 29, 118-121.

Kleinke, C. L., Bustos, A. A., Meeker, F. B., \& Staneski, R. A. (1973). Effect of selfattributed and other-attributed gaze on interpersonal evaluations between males and female. Journal of Experimental Social Psychology, 9, 154-163.

Koenig, A., \& Eagly, A. (2005). Stereotype threat in men on a test of social sensitivity. Sex Roles, 52, 489-496. 
Korsch, B. M., Gozzi, E. K., \& Francis, V. (1968). Gaps in doctor-patient communication: Doctor-patient interaction and patient satisfaction. Pediatrics, 42, $855-871$.

Kramer, C. (1977). Perceptions of female and male speech. Language and Speech, 20, $151-161$.

Krippendorff, K. (2004). Content analysis. Thousand Oaks, CA: Sage Publications.

Krupat, E., Frankel, R., Stein, T., \& Irish, J. (2006). The four habits coding scheme: Validation of an instrument to assess clinicians' communication behavior. Patient Education and Counseling, 62, 38-45.

Lakoff, R. (1975). Language and woman's place. New York: Harper \& Row.

Laljee, M., \& Cook, M. (1975). Anxiety and ritualized speech. British Journal of Psychology, 66, 299-306.

Larew, C., Lessans, S., Spunt, D., Foster, D., \& Covington, B. G. (2006). Innovations in clinical simulation: Application of Benner's theory in an interactive patients care simulation. Nursing Education Perspectives, 27, 16-21.

Laroche, E., \& Livner, H. (1983). Regressional analysis of attitudes toward male nurses. The Journal of Psychology, 113, 67-71.

Leininger, M. (1984). Care: The essence of nursing and health. Thorofare, NJ: Charles S. Black.

Leininger, M. (1991). Culture care diversity and universality: A theory of nursing. New York: National League for Nursing Press.

Lester, G. W., \& Smith, S. G. (1993). Listening and talking to patients: A remedy for malpractice suits? Western Journal of Medicine, 158, 268-272. 
Levy, B. (1996). Improving memory in old age through implicit self-stereotyping. Journal of Personality and Social Psychology, 71, 1092-1107.

Ley, P. (1982). Satisfaction, compliance and communication. British Journal of Clinical Psychology, 21, 241-254.

Leyens, J. Desert, M., Croizet, J., \& Darcis, C. (2000). Stereotype threat: Are lower status and history of stigmatization preconditions of stereotype threat? Personality and Social Psychology Bulletin, 26, 1189 - 1199.

Lipley, N. (2000). War on attrition. Nursing Standard, 15, 12.

Lippmann, W. (1922). Public opinion. New York: The Free Press.

Lippmann, W. (1953). Stereotypes. In B. Berelson \& M. Janowitz (Eds.), Reader in public opinion and communication. New York, NY: The Free Press.

Lister, J. (1971). The doctor's changing role. New England Journal of Medicine, 285, 790-792.

Lo, R., \& Brown, R. (1999). Perceptions of nursing students on men entering nursing as a career. Australian Journal of Advanced Nursing, 17, 36-41.

Lord, C. G., \& Saenz, D. S. (1985). Memory deficits and memory surfeits: Differential cognitive consequences of tokenism for tokens and observers. Journal of Perosnality and Social Psychology, 49, 918-926.

Lord, C. G., Saenz, D. S., \& Godfrey, D. K. (1987). Effects of perceived scrutiny on participant memory for social interactions. Journal of Experimental Social Psychology, 23, 498-517.

Loughrey, M. (2008). Just how male are male nurses.? Journal of Clinical Nursing, 17, 1327-1334. 
Lupton, B. (2006). Explaining men's entry into female-concentrated occupations: Issues of masculinity and social class. Gender, Work and Organization, 13, 103-128.

Lynch, M. D., \& May, L. (1977). Some effects of heightening anxiety levels on writing performance of students with different levels of creativity and prior anxiety. Paper presented to the American Educational Research Association.

MacPhail, J. (1996). Men in nursing. In J. R. Ken \& J. MacPhail (Eds.), Canadian nursing: Issues and perspectives ( ${ }^{\text {rd }}$ ed., pp. 74-81). St. Louis: Mosby-Yearbook.

Madden, B. P. (1990). The hybrid model for concept development: Its value for the study of therapeutic alliance. Advances in Nursing Science, 12, 75-87.

Mael, F., \& Ashforth, B. (1992). Alumni and their alma mater: A partial test of the reformulated model of organizational identification. Journal of Organizational Behavior, 13, 103-123.

Mahalik, J. R., Locke, B. D., Ludlow, L. H., Diemer, M. A., Gottfried, M., Scott, R. P. J., \& Freitas, G. (2003). Development of the Conformity to Masculine Norms Inventory. Psychology of Men \& Masculinity, 4, 3-25.

Mahalik, J. R., Morray, E. B., Coonerty-Femiano, A., Ludlow, L. H., Slattery, S. M., \& Smiler, A. (2005). Development of the conformity to feminine norms inventory. Sex Roles, 52, 417-435.

Major, B., Spencer, S. J., Schmader, T., Wolfe, C., \& Crocker, J. (1998). Coping with negative stereotypes about intellectual performance: The role of psychological disengagement. Personality and Social Psychology Bulletin, 24, 34-50. 
Marteau, T. M., \& Bekker, H. (1992). The development of a six-item short form of the state scale of the Spielberger State-Trait Anxiety Inventory. British Journal of Clinical Psychology, 31, 301-306.

Martin, J. N., \& Craig, R. T. (1983). Selected linguistic sex differences during initial social interactions of same-sex and mixed-sex dyads. The Western Journal of Speech Communication, 47, 16-28.

Marvel, M. K., Doherty, W. J., \& Weiner, E. (1998). Medical interviewing by exemplary family physicians. Journal of Family Practice, 47, 343-348.

Massey, D. S., \& Fischer, M. J. (2005). Stereotype threat and academic performance: New findings from a racially diverse sample of college freshmen. Du Bois Review, 2, 45-67.

McCance, T. V., McKenna, H. P., \& Boore, J. R. P. (1999). Caring: Theoretical perspectives of relevance to nursing. Journal of Advanced Nursing, 30, 13881395.

McCroskey, J. C. (1978). Oral communication apprehension: A summary of recent theory and research. Human Communication Research, 32, 274-301.

McCroskey, J. C. (1982). Oral communication apprehension: A reconceptualization. Communication Yearbook, 6, 136-170.

McCroskey, J. C., Beatty, M. J., Kearney, P., \& Plax, T. G. (1985). The content validity of the PRCA-24 as a measure of communication apprehension across communication contexts. Communication Quarterly, 33, 165-173.

McDonald, L. (2010). Florence Nightingale: The Crimean War: Collected works of Florence Nightingale. Ontario, Canada: Wilfrid Laurier University Press. 
McDougall, G. (1997). Caring - a masculine perspective. Journal of Advanced Nursing, $25,809-813$.

McGillcuddy-De Lisi, A. V., \& De Lisi, R. (2002). Biology, society, and behavior: The development of sex differences in cognition. Greenwich, CT: Ablex/Greenwood.

McGlone, M., \& Aronson, J. (2006). Stereotype threat, identity salience, and spatial reasoning. Journal of Applied Developmental Psychology, 27, 486-493.

McGlone, M. S., Aronson, J., \& Kobrynowicz, D. (2006). Stereotype threat and the gender gap in political knowledge. Psychology of Women Quarterly, 30, 392-398.

McGlone, M. S., Kobrynowicz, D., \& Aronson, J. (1999, July). Ground for stereotype threat: Social identity and test difficulty. Paper presented at the Symposium on Stereotype Threat at the Annual Meeting of the European Association of Experimental Social Psychology, Oxford University.

McGlone, M. S., \& Pfiester, R. A. (in press). Stereotype threat and the evaluative context of communication. Communication Research.

McKay, P. F., Doverspike, D., Bowen-Hilton, D., \& Martin, Q. D. (2002). Stereotype threat effects on the Raven Advanced Progressive Matrices of African Americans. Journal of Applied Social Psychology, 32, 767-787.

McLaughlin, K., Muldoon, O. T., \& Moutray, M. (2010). Gender, gender roles and completion of nursing education: A longitudinal study. Nurse Education Today, 30, 303-307.

McMillan, J. R., Clifton, A. K., McGrath, D., \& Gale, W. S. (1977). Women's language: Uncertainty or interpersonal sensitivity and emotionality? Sex Roles, 3, 545-559. 
McRae, M. J. (2003). Men in obstetrical nursing: Perceptions of the role. The American Journal of Maternal/Child Nursing, 28, 167-173.

Meadus, R. J. (2000). Men in nursing: Barriers to recruitment. Nursing Forum, 35, 5-12.

Meadus, R. J., \& Twomey, J. C. (2007). Men in nursing: Making the right choice. Canadian Nurse, 103, 13-16.

Meadus, R. J., \& Twomey, J. C. (2011). Men student nurses: The nursing education experience. Nursing Forum: An Independent Voice for Nursing, 46, 269-279.

Mehrabian, A., \& Epstein, N. (1972). A measure of emotional empathy. Journal of Personality, 40, 525-543.

Miller, A. G. (1982). In the eye of the beholder: Contemporary issues in stereotyping. New York, NY: Praeger.

Miller, L., \& Budd, J. (1999). The development of occupational sex-role stereotypes, occupational preferences and academic subject preferences in children at ages, 8, 12, and 16. Educational Psychology, 19, 17-35.

Miller, L., Neathey, F., Pollard, E., \& Hill, D. (2004). Occupational segregation, gender gaps and skill gaps. Equal Opportunities Commission Working Paper, series 15.

Milligan, F. (2001). The concept of care in male nurse work: An ontological hermeneutic study in acute hospitals. Journal of Advanced Nursing, 35, 7-16.

Morse, J. M., Solberg, S. M., Neander, W. L., Bottorff, J. L., \& Johnson, J. L. (1990). Concepts of caring and caring as concept. Advances in Nursing Science, 13, 1-14.

Moyer, C. A., Stern, D. T., Dobias, K. S., Cox, D. T., \& Katz, S. J. (2002). Bridging the electronic divide: Patient and provider perspective on e-mail communication in primary care. American Journal of Managed Care, 8, 427-433. 
Mulac, A., Lundell, T. L., \& Bradac, J. J. (1986). Male/female language differences and attributional consequences in a public speaking situation: Toward an explanation of the gender-likened language effect. Communication Monographs, 53, 115-129.

Mulholland, J., Anionwu, E. N., Atkins, R., Tappern, M., \& Franks, P. (2008). Diversity attrition, and transition into nursing. Journal of Advanced Nursing, 64, 49-59.

National League for Nursing (NLN). (2007). Nursing data review academic year 2007 baccalaureate, associate degree, and diploma programs. New York: Author.

Nelson, R., \& Belcher, D. (2006). Men in nursing: Discrimination and stereotypes still keep many from joining nursing ranks. The American Journal of Nursing, 106, 25-26.

Newman, M. L., Groom, C. J., Handelman, L. D., \& Pennebaker, J. W. (2008). Gender differences in language use: An analysis of 14,000 text samples. Discourse Processes, 45, 211-236.

Norman, L. D., Donelan, D., Buerhaus, P. I., Willis, G., Williams, M., Ulrich, B., \& Dittus, R. (2005). The older nurse in the workplace: Does age matter? Nursing Economics, 23, 282-289.

Nguyen, H. H. D., O’Neal, A., \& Ryan, A. M. (2003). Relating test-taking attitudes and skills and stereotype threat effects to the racial gap in cognitive ability test performance. Human Performance, 16, 261-293.

O’Brien, L. T., \& Crandall, C. S. (2003). Stereotype threat and arousal: Effects on women's math performance. Personality and Social Psychology Bulletin, 29, 782789. 
O’Dowd, D. D., \& Beardslee, D. C. (1967). Development and consistency of student images of occupations. Washington, D.C.: Department of Health, Education, and Welfare.

O’Lynn, C. E. (2004). Gender-based barriers for male students in nursing education programs: Prevalence and perceived importance. Journal of Nursing Education, $43,229-236$.

O’Lynn, C. E., \& Tranbarger, R. E. (2007). Men in nursing: History, challenges, and opportunities. New York, NY: Springer.

O’Neil, J. M. (1981a). Male sex role conflicts, sexism, and masculinity: Psychological implications for men, women, and the counseling psychologist. The Counseling Psychologist, 9, 61-80.

O'Neil, J. M. (1981b). Patterns of gender role conflict and strain: Sexism and fear of femininity in men's lives. The Personnel and Guidance Journal, 60, 203210.

Oliver, M. B. (1999). Caucasian viewers' memory of Black and White criminal suspects in the news. Journal of Communication, 49, 46-60.

Oliver, M. B., \& Fonash, D. (2002). Race and crime in the news: Whites' identification and misidentification of violent and nonviolent criminal suspects. Media Psychology, 4, 137-156.

Okrainec, G. (1990). Males in nursing. Historical perspectives and analysis. Alberta Association of Registered Nurses, 46, 6-8.

Oppenheimer, V. K. (1968). The sex labeling of jobs. Industrial Relations, 7, 219-234. 
Osborne, J. W. (1995). Academics, self-esteem, and race: A look at the underlying assumptions of the disidentification hypothesis. Personality and Social Psychology Bulletin, 21, 449-455.

Osborne, J. W. (2001). Testing stereotype threat: Does anxiety explain race and sex differences in achievement? Contemporary Educational Psychology, 26, 291-310.

Palomares, N. A. (2008). Explaining gender-based language use: Effects of gender identity salience on references to emotion and tentative language in intra- and intergroup contexts. Human Communication Research, 34, 263-286.

Palomares, N. A. (2004). Gender schemacity, gender identity salience, and gender-linked language use. Human Communication Research, 30, 556-588.

Palomares, N. A. (2009). Women are sort of more tentative than men, aren't they? How men and women use tentative language differently, similarly and counter stereotypically as a function of gender salience. Communication Research, 36, 538560.

Parent, M. C., \& Moradi, B. (2010). Confirmatory factor analysis of the conformity to feminine norms inventory and development of an abbreviated version: The CFNI45. Psychology of Women Quarterly, 34, 97-109.

Parent, M. C., \& Moradi, B. (2009). Confirmatory factor analysis of the conformity to masculine norms inventory and development of the conformity to masculine norms inventory-46. Psyschology of Men \& Masculinity, 10, 175-189.

Paterson, B. L., Tschikota, S., Crawford, M., Saydak, M., Venkatesh, P., \& Aronowitz, T. (1996). Learning to care: Gender issues for male nursing students. Canadian Journal of Nursing, 28, 25-39. 
Paterson, J. G., \& Zderad, L. T. (1976). Interpersonal relations in nursing: A conceptual frame of reference. New York: G. P. Putnam.

Patterson, M. L., \& Ritts, V. (1997). Social and communicative anxiety: A review and meta-analysis. In B. R. Burleson (Ed.), Communication yearbook 20 (pp. 262-303). Thousand Oaks, CA: Sage.

Peeke, A. P, Monroe, C. W., \& Hulit, B. E. (1968). They physician's obligation to his patients. Journal of the American Medical Association, 204, 88-90.

Peffley, M., Shields, T., \& Williams, B. (1996). The intersection of race and crime in television news stories: An experimental study. Political Communication, 13, 309327.

Penchansky, R., \& Macnee, C. (1994). Initiation of medical malpractice suits: A conceptualization and test. Medical Care, 32, 813-831.

Pennebaker, J. W., Booth, R. J., \& Francis, M. E. (2007). Linguistic Inquiry and Word Count: LIWC 2007. Austin, TX: LIWC (www.liwc.net).

Peplau, H. E. (1952). Interpersonal relations in nursing. New York: Putnam.

Peplau, H. E. (1987). Interpersonal constructs for nursing practice. Nurse Education Today, 7, 201-208.

Peplau, H. E. (1997). Peplau's theory of interpersonal relations. Nursing Science Quarterly, 10, 162-167.

Perkins, T. E. (1979). Rethinking stereotypes. In M. Barrett, P. Corrigan, A. Kuhn, \& J. Wolff (Eds.), Ideology and cultural production (pp. 135-159). London: Croom Helm.

Pfeil, U., \& Zaphiris, P. (2007). Patterns of empathy in online communication. In 
Proceeding of CHI 2007 - The ACM Conference on human factors in computing systems (pp. 919-928). New York: ACM Press.

Pittman, E. (2005). Luther Christman: A maverick nurse - A nursing legend. Victoria, BC Canada: Tafford.

Poliafico, J. K. (1998). Nursing's gender gap. RN, 61, 39-42.

Porter, M. A. (2010). Third-party imagined interactions: Expanding mental imagery to the experience of others' communication. Imagination, Cognition, \& Personality, $30,147-169$.

Preacher, K. J., \& Hayes, A. F. (2008). Asymptotic and resampling strategies for assessing and comparing indirect effects in multiple mediator models. Behavior Research Methods, 40, 879-891.

Preacher, K. J., Rucker, D. D., \& Hayes, A. F. (2007). Addressing moderated mediation hypotheses: Theory, methods, and prescriptions. Multivariate Behavioral Research, 42, 185-227.

Pryhmachuk, S., Easton, K., \& Littlewood, A. (2008). Nurse education: Factors associated with attrition. Journal of Advanced Nursing, 65, 149-160.

Quinn, D. M., \& Spencer, S. J. (2001). The interference of stereotype threat with women's generation of mathematical problem-solving strategies. Journal of Social Issues, 57, 55-71.

Ralston, J. D., Rutter, C. M., Carrell, D., Hecht, J., Rubanowice, D., \& Simon, G. E. (2009). Patient use of secure electronic messaging within a shared medical record: A cross-sectional study. Journal of General Internal Medicine, 24, 349-355. 
Ramos, M. C. (1992). The nurse-patient relationship: Theme and variations. Journal of Advanced Nursing, 17, 496-506.

Reed, S. A., Keerie, N., \& Palomares, N. A. (2003). Language, gender salience, and social influence. Journal of Language and Social Psychology, 22, 210-233.

Reynolds, A. (2009). Patient-centered care. Radiologic Technology, 81, 133-147.

Riffe, D., Stephen, L., \& Fico, F. G. (2005). Analyzing media messages: Using quantitative content analysis in research. Mahwah, NJ: Erlbaum.

Roach, S. (1984). Caring: The human mode of being: Implications for nursing. Toronto: University of Toronto Press.

Rochlen, A. B., Good, G. E., \& Carver, T. A. (2009). Predictors of gender-related barriers, work, and life satisfaction among men in nursing. Psychology of Men \& Masculinity, 10, 44-56.

Rogan, F., \& Timmins, F. (2004). Improving communication in day surgery setting. Nursing Standard, 19, 37-42.

Rosenkrantz, P., Vogel, S., Bee, H., Broverman, I., \& Broverman, D. M. (1968). Sex-role stereotypes and self-concepts in college students. Journal of Consulting and Clinical Psychology, 32, 287-295.

Roter, D. (1989). Which facets of communication have strong effects on outcome: A meta-analysis. In M. Stewart \& D. Roter (Eds.), Communicating with medical patients (pp. 183-197). Newbury Park, CA: Sage.

Rudman, L. A., \& Kilianski, S. E. (2000). Implicit and explicit attitudes toward female authority. Personality and Social Psychology Bulletin, 26, 1315-1328. 
Ryan, E. B., Giles, H., Bartolucci, G., \& Henwood, K. (1986). Psycholinguistic and social psychological components of communication by and with the elderly. Language \& Communication, 6, 1-24.

Ryckman, D. B., \& Peckham, P. (1987). Gender differences in attributions for success and failure situations across subject areas. Journal of Educational Research, 81, $120-125$.

Sastre, M. T. M., Fouquereau, E., Igier, V., Salvatore, N., \& Mullet, E. (2000). Perception of occupational gender typing: A replication on European Samples of Shinar's (1975) and Beggs and Doolittle's (1993) studies. Journal of Applied Social Psychology, 30, 430-441.

Scarr, S., \& Carter-Saltzman, L. (1982). Genetics and intelligence. In J. L. Fuller \& E. C. Simmel (Eds.), Behavior genetics (pp. 217-335). Hillsdale, NJ: Erlbaum.

Schmader, T. (2007). An integrated model of stereotype threat effects on performance. Unpublished manuscript. University of Arizona.

Schmader, T. (2002). Gender identification moderates stereotype threat. Journal of Experimental Social Psychology, 37, 32-44.

Scotto, C. J. (2003). A new view of caring. Journal of Nursing Education, 42, 289-291.

Schneider, D. J. (2004). The psychology of stereotyping. New York: Guilford Press.

Selfe, S. A., Matthews, Z., \& Stones, R. W. (1998). Factors influencing outcome in consultations for chronic pelvic pain. Journal of Women's Health, 7, 1041-1048.

Severines, S., Dam, G. T. (2012). Leaving college: A gender comparison in male and female-dominated programs. Research in Higher Education, 53, 453-470. 
Shapiro, E. (2007). Drag kinging and the transformation of gender identities. Gender \& Society, 21, 250-271.

Shattell, M. (2004). Nurse-patient interaction: A review of the literature. Journal of Clinical Nursing, 13, 714-722.

Sheldon, L. K., Barrett, R., \& Ellington, L. (2006). Difficult communication in nursing. Journal of Nursing Scholarship, 38, 141-147.

Shellenbargen, T. (1993). Eliminating sexism in the classroom. Nurse Educator, 18, 1720.

Shih, M., Pittinsky, T. L., \& Ambady, N. (1999). Stereotype susceptibility: Identity salience and shifts in quantitative performance. Psychological Science, 10, 80-83.

Shinar, E. H. (1975). Sexual stereotypes of occupations. Journal of Vocational Behavior, 7, 99-111.

Silver, B. D., Anderson, B. A., \& Abramson, P. R. (1986). Who overreports voting? American Political Science Review, 80, 613-624.

Simpson, M. (1985). How to use role-play in medical teaching. Medical Teacher, 7, 7582.

Sittig, D. F., King, S., \& Hazlehurst, B. L. (2001). A survey of patient-provider e-mail communication: What do patients think? International Journal of Medical Informatics, 61, 71-80.

Smith, J. S. (2006). Exploring the challenges for nontraditional male students transitioning into a nursing program. Journal of Nursing Education, 45, 263-269. 
Smith, J. L., \& White, P. H. (2001). Development of the domain identification measure: A tool for investigating stereotype threat effects. Educational and Psychological Measurement, 61, 1040-1057.

Sorrentino, R. M., \& Boutillier, R. G. (1975). The effect of quantity and quality of verbal interaction on ratings of leadership ability. Journal of Experimental Social Psychology, 11, 403-411.

Spence, J. T., Helmreich, R., \& Stapp, J. (1974). The personal attributes questionnaire: A measure of sex-role stereotypes and masculinity-femininity. JSAS Catalog of Selected Documents in Psychology, 4, 43-44.

Spencer, S., Steele, C., \& Quinn, D. (1999). Stereotype threat and women's math performance. Journal of Experimental Social Psychology, 35, 4-28.

Spichiger, E., Wallhagen, \& Benner, P. (2005). Nursing as a caring practice from a phenomological perspective. Scandinavian Journal of Caring Science, 19, 303309.

Spielberger, C. D., Gorsuch, R. L., \& Lushene, R. (1970). STAI manual. Palo Alto, CA: Consulting Psychologists Press.

Spratley, E., Johnson, A., Sochalski, J., Fritz, M., \& Spencer, W. (2002). The registered nurse population: Findings from the national sample survey of registered nurses: March 2000. Washington, D.C.: Health Resources and Service Administration.

Squires, T. (1995). Men in nursing. $R N$, 58, 26-28.

Stangor, C. (2009). The study of stereotyping, prejudice, and discrimination within social psychology: A quick history of theory and research. In T.D. Nelson (Ed.), 
Handbook of prejudice, stereotyping, and discrimination (pp. 1-22). New York: Psychology Press.

Steadman, R. H., Coates, W. C., Huang, Y. M., Matevosian, R., Larmon, B. R., McCullough, L., \& Ariel, D. (2006). Simulation-based training is superior to problem-based learning for the acquisition of critical assessment and management skills. Critical Care Medicine, 34, 151-157.

Steele, C. M. (1997). A threat in the air: How stereotypes shape intellectual identity and performance. American Psychologist, 52, 613-629.

Steele, C. M., \& Aronson, J. (1995). Stereotype threat and the intellectual test performance of African Americans. Journal of Personality and Social Psychology, 69, 797-811.

Steele, C. M., Spencer, S. J., \& Aronson, J. (2002). Contending with group image: The psychology of stereotype and social identity threat. In M. Zanna (Ed.), Advances in experimental social psychology (Vol. 34, pp. 379-440). San Diego, CA: Academic Press.

Stein, R. T., \& Heller, T. (1979). An empirical analysis between leadership status and participation rates reported in the literature. Journal of Personality and Social Psychology, 37, 1993-2002.

Stewart, I. M., \& Austin, A. L. (1962). A history of nursing (5th ed.). New York: G.P. Putman's Sons.

Stott, A. (2007). Exploring factors affecting attrition of male students from an undergraduate nursing course: A qualitative study. Nurse Education Today, 27, $325-332$. 
Streubert, H. J. (1994). Male nursing students: Perceptions of clinical experience. Nurse Educator, 19, 29-32.

Strotbeck, F. (1951). Husband-wife interaction over revealed differences. American Sociological Review, 16, 468-473.

Studer Group. (2007). AIDET SM: Five fundamentals of patient communication [video series]. Gulf Breeze, FL: Fire Starter Publishing.

Tajfel, H. (1981). Human groups and social categories: Studies in social psychology. Cambridge: Cambridge University Press.

Tajfel, J., \& Turner, J. C. (1986). The social identity theory of intergroup behavior. In S. Worchel \& W. G. Austin (Eds.), Psychology of intergroup relations $\left(2^{\text {nd }}\right.$ ed., pp. 7-24). Chicago: Nelson-Hall.

Thayer, J. F., \& Johnson, D. H. (2000). Sex differences in judgement of facial affect: A multivariate analysis of recognition errors. Scandinavian Journal of Psychology, $41,243-246$.

Thernstrom, A., \& Thernstrom, S. (2003). No excuses: Closing the racial gap in learning. New York, NY: Simon \& Shuster Paperbacks.

Thomas, H. (1993). A theory explaining sex differences in high mathematical ability has been around for some time. Behavioral and Brain Sciences, 16, 187-215.

Tracey, C., \& Nicholl, H. (2007). The multifaceted influence of gender in career progress in nursing. Journal of Nursing Management, 15, 677-682.

Tyson, N. D. (1991, May). Ph.D. convocation address. Speech presented at St. Paul's Chapel, Columbia University, New York City. 
Turner, J. C., Hogg, M. A., Oakes, P. J., Reicher, S. D., \& Wetherell, M. S. (1987). Rediscovering the social group: A self-categorization theory. Oxford: Basil Blackwell.

U.S. Bureau of Labor Statistics. (2012). Occupational employment and wages: Registered nurses. Retrieved from http://www.bls.gov/oes/current/oes291111.htm

U.S. Census Bureau. (2013). Male nurses becoming more commonplace. Retrieved from http://www.census.gov/newsroom/releases/archives/cb13-32.html

U.S. Department of Health and Human Services, Health Resources and Service Administration. (2006). The registered nurse population: Findings from the March 2004 national sample survey of registered nurses. Rockville, MD: Author.

U.S. Department of Health and Human Services, Health Resources and Service Administration (2010). The registered nurse population: Initial findings from the 2008 national sample survey of registered nurses. Rockville, MD: Author.

University of Iowa, College of Nursing. (2012). Student organizations: University of Iowa Men in Nursing (UI MiN). Retrieved from http://www.nursing.uiowa.edu/current-students/uiowa-men-in-nursing

University of Pennsylvania, School of Nursing. (2012). Male association of nursing at the University of Pennsylvania (MAN-UP). Retrieved from http://www.nursing.upenn.edu/ manup/Pages/About.aspx

University of Texas at Austin, School of Nursing. (2012). LAMN: The Longhorn association for men in nursing. Retrieved from http://www.utexas.edu/nursing/lamn/index.html 
University of Washington, School of Nursing. (2012). Men in nursing. Retrieved from http:// nursing.uw.edu/node/564

Villeneuve, M. J. (1994). Recruiting and retaining men in nursing: A review of the literature. Journal of Professional Nursing, 10, 217-228.

Vincent, C., Young, M., \& Phillips, A. (1994). Why do people sue doctors? A study of patients and relatives taking legal action. Lancet, 343, 1609-1613.

Wagner, J. A., Pfeiffer, C. A., \& Harrington, K. L. (2011). Evaluation of online instruction to improve medical and dental students' communication and counseling skills. Education \& Health Professions, 34, 383-397.

Walther, J. B. (1996). Computer-mediated communication: Impersonal, interpersonal, and hyperpersonal interaction. Communication Research, 23, 1-43.

Ware, J. E., Davies-Avery, A., \& Stewart, A. L. (1978). The measurement and meaning of patient satisfaction: A review of the recent literature. Health and Medical Care Services Review, 1, 1-15.

Watson, J. (1985). Nursing: The philosophy and science of caring. Boulder, CO: Colorado Associated University Press.

White, M. J., \& White, G. B. (2006). Implicit and explicit occupational gender stereotypes. Sex Roles, 55, 259-266.

Wickramasinghe, N. S., Fadlalla, A. M. A., Geisler, E., \& Schaffer, J. L. (2005). A framework for assessing e-health preparedness. International Journal of Electronic Healthcare, 1, 316-334. 
Wilkinson, S., Robets, A., \& Aldridge, J. (1998). Nurse, patient communication in palliative care: An evaluation of a communication skills programme. Palliative Medicine, 12, 13-22.

Williams, C. L. (1995). Still a man's world: Men who do women's work. Berkeley, CA: University of California Press.

Williams, D. (2002). Looking for a few good men. Retrieved from www.minoritynurse.com

Williams, J. E., \& Best, D. L. (1990). Measuring sex stereotypes: A thirty-nation study. Beverly Hills, CA: Sage.

Williams, L. S., \& Villemez, W. J. (1993). Seekers and finders: Male entry and exit in female-dominated jobs. In C. L. Williams (Ed.), Doing women's work: Men in nontraditional occupations (pp. 64-90). London: Sage.

Wilson, B. (1997). Men in American nursing history. Retrieved from http://www.geocities.com/ Athens/Forum/6011/

Wilson, G. (2005). The experience of males entering nursing: A phenomenological analysis of professionally enhancing factors and barriers. Contemporary Nurse, $20,221-233$.

Wolfenden, J. (2011). Men in nursing. The Internet Journal of Allied Health Sciences and Practice, 9, 1-6.

Yang, C. I., Gau, M. L., Shiau, S. J., Hu, W. H., \& Shih, F. J. (2004). Professional career development for male nurses. Journal of Advanced Nursing, 48, 642-650.

Yurkovich, D. (2006, August-September). Where the boys aren't: Gender disparity and the nursing profession. MODRN Nurse Magazine, pp. 26-31. 
Ziv, A., Small, S. D., \& Wolpe, P. R. (2000). Patient safety and simulation-based medical education. Medical Teacher, 22, 489-495.

Ziv, A., Wolpe, P. R., Small, S. D., \& Glick, S. (2003). Simulation-based medical evaluation: An ethical imperative. Academic Medicine, 78, 783-788. 


\section{VITA}

Andrew Craig Tollison graduated from Karns High School, Knoxville, Tennessee in 1999. He is an Honor's graduate from the University of Tennessee (2003) with a Bachelor of Science degree in Broadcasting. Andrew also earned his Master of Science degree in Communication Studies from the University of Tennessee (2005). Upon completion of his Masters degree, Andrew taught in the School of Communication Studies at the University of Tennessee for two years. In August 2007, he moved to Austin to begin and complete his doctoral work in the Department of Communication Studies at the University of Texas at Austin.

Email Address: actollison@gmail.com

This manuscript was typed by the author. 